\title{
Game Balance Designed structure and consumer agency in an online game
}

\section{Elizabeth Haynes}

A thesis submitted to the Victoria University of Wellington in fulfilment of the requirements from the degree of Master of Arts in Cultural Anthropology

Victoria University of Wellington 2013 


\begin{abstract}
Massively multi-player online role-playing games (MMORPGs) attract millions of people every year and are now a major industry. Using the internet, these games connect players and give them goals to pursue within virtual worlds. This thesis examines the early life of one such game, the North American version of TERA, based on participant observation on a player vs. player server. TERA's players met and interacted within a virtual game world controlled by the company which developed the game, and although players constructed their own social groups and factions within this world they were constrained by software that they could not change. Everything from the combat rules to the physics of the environment was designed, and players could only take actions that were accounted for and allowed by that design.
\end{abstract}

However, TERA launched as one of many available MMORPGs which were competing for the attention of the same audience. Its players tended to be experienced and well-informed about the genre, and used their knowledge to evaluate and critique TERA both privately and in public forums. Aware that game companies' chief concern was for profit, players exercised agency by embracing a consumer identity and pressuring developers in their own commercial terms. To retain players' loyalty and continue receiving their fees, companies were obliged to appease their customers. This allowed players to see the game world develop and change in accordance with their desires despite the fact that they lacked the access or the expertise to change it themselves. I link this approach to agency to the rise of consumer movements in capitalist societies, and show how the virtual world of TERA can serve as an example for other situations in the physical world where contemporary technologies are used to both enable and constrain agency. 


\section{Acknowledgements}

Thanks must first go to Catherine Trundle, my supervisor, whose advice and guidance have been invaluable. Our discussions, ranging on and off topic as they did, enriched this thesis greatly; without her, it might have just been about video games. Lorena Gibson's advice was also very much appreciated, and helped me to take the technicalities of the virtual world and present the whole as a little more human.

Jaron Peek's unfailing support played a big part in getting me through this project without major trauma. Thank you, Jaron, for always being there and willing to listen to me rant! Clare Barcazk also got her share of ranting, and I'd like to thank her for stimulating discussions and occasional commiseration.

Lara Bell helped to get me on my feet at the beginning and was a great office-mate thank you for the discussion and the encouragement. Jared Walton's proof-reading was a great help at just the right time, and was appreciated. My fellow anthropology postgrads were good company and offered good advice throughout.

Finally, thank you to the TERA players who participated in this research, the guild here called Bellicus and especially the players referred to as Jelena and Elleon. From half a world away, you not only accepted me but accommodated me and helped me out in numerous ways. I suspect I could not have fallen in with a better guild.

I dedicate this work to John Haynes, my father, who didn't live to see its completion but probably would have been pretty amused that I managed to get a degree by playing video games. Dad, you are missed. 


\section{Table of Contents}

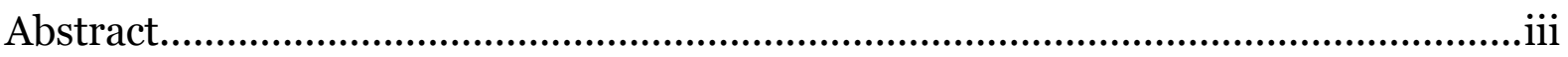

Acknowledgements ................................................................................................... iv

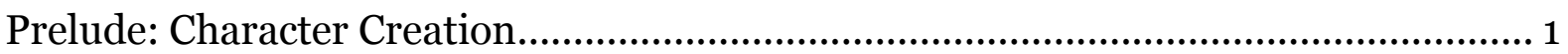

Chapter One - Introduction ........................................................................... 5

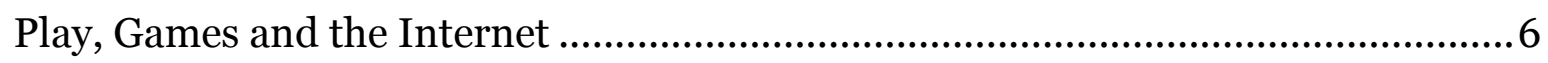

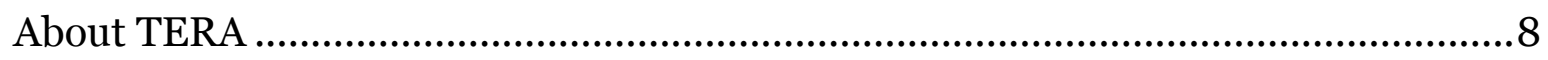

Structure, Agency and Practice...................................................................................11

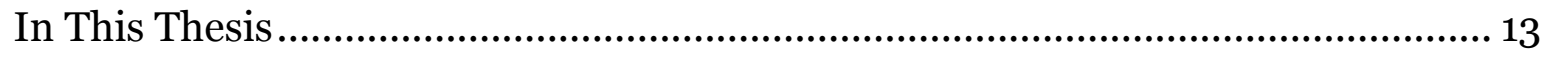

Chapter Two - Methods and Ethics …………………...................................... 17

The Researcher ………............................................................................................. 17

Methodological Decisions ........................................................................................... 19

Ethnographic Research........................................................................................ 21

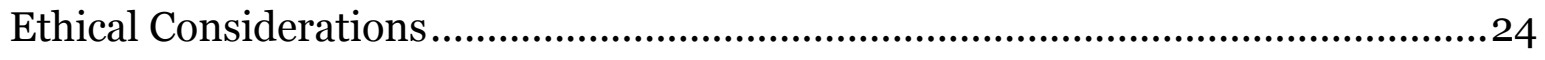

Interlude: Sinestral Manor...................................................................................29

Chapter Three - The Life Cycle of a Game ……………........................................33

Temporality, History and Migration Online ………................................................... 33

The Road to Release..............................................................................................

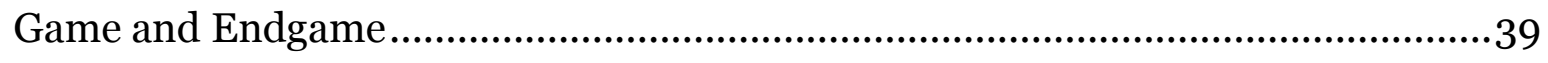

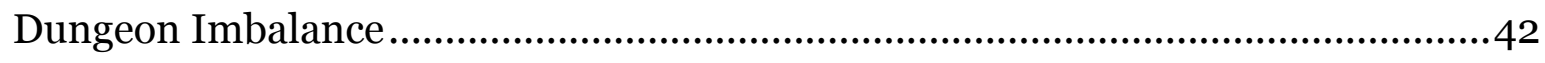

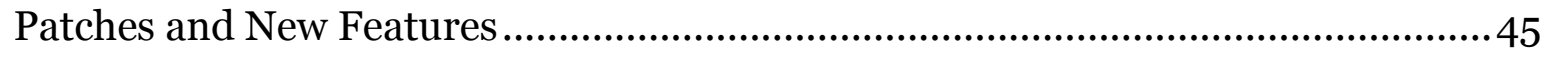

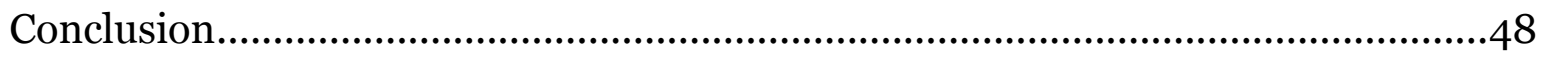

Interlude: Guild Conversation .................................................................................... 51

Chapter Four - The Life-Cycle of a Guild …………………………………....53

Formation and Leadership ...................................................................................53

Tensions and Teamwork.................................................................................56 
Fission

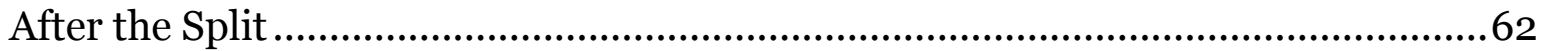

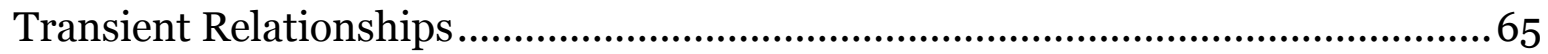

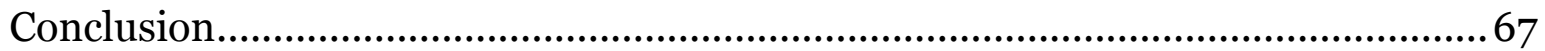

Chapter Five - Fairness and Legitimacy .............................................69

Expectations of Fairness: Balance .............................................................69

Game Balance and Social Power: The Lancer ............................................... 73

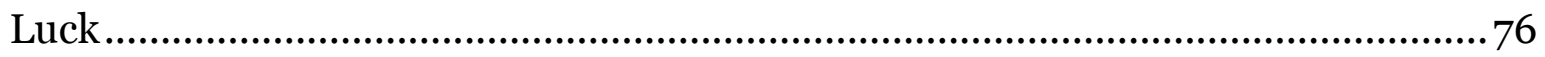

The Random Number Gods ...................................................................... 78

Legitimate and Illegitimate Killing ............................................................... 81

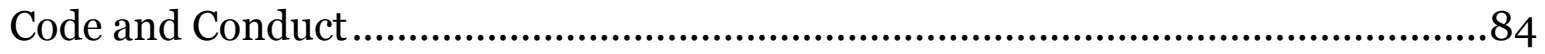

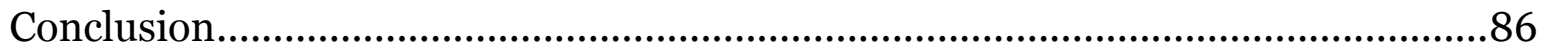

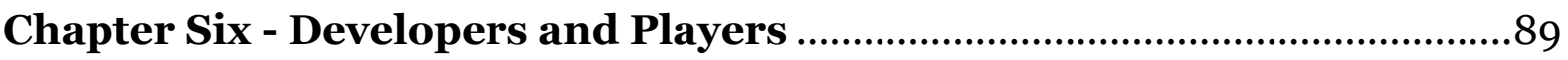

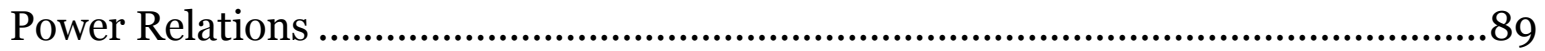

The Other Meta-Game ............................................................................93

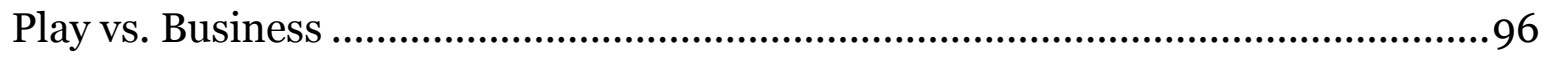

Virtual Structures.................................................................................99

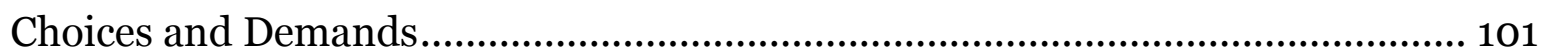

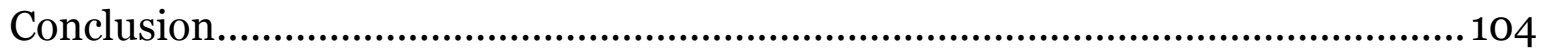

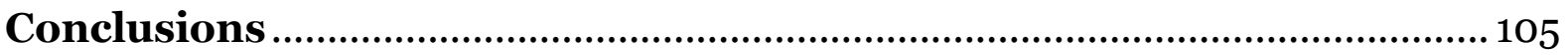

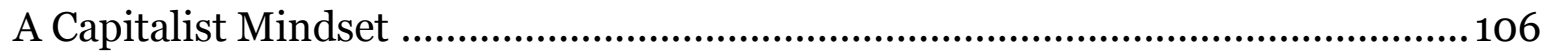

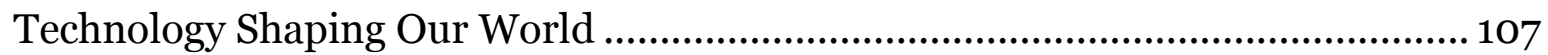

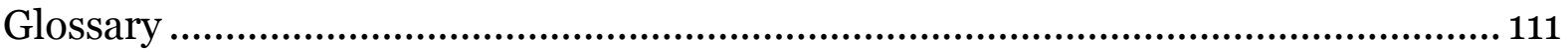

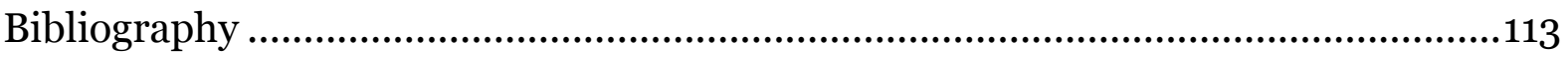

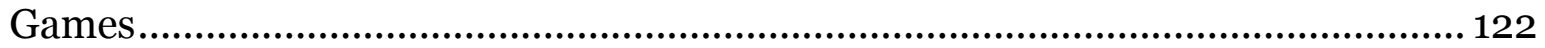




\section{Prelude: Character Creation}

(Based on field notes from April 15 $5^{\text {th }}$ 2012)

It's early character creation weekend - a time for those of us who pre-purchased the game to make one character who will be permanent on our accounts, even though we can't use them yet. Our characters will be our avatars, the virtual bodies with which we are identified and which we use to play the game. Players want to secure the names they want, and to have some time to craft the perfect appearance.

This will be the first time I have loaded the game client from the desktop of my computer. I enter my account details, and after a short while it directs me to a title screen. I am treated to one of TERA's typically bright and surreal landscapes, with a typically scantily-clad woman in the foreground. She faces away from me, and she has horns - a 'castanic', like the game's poster girl. The first option provided is to select a server. There are eleven, each leading to a copy of the game world with a different group of players; a server can only handle so many people accessing it at once, so we will be spread out. Luckily I already know where I need to base my first character - my guild's leaders have announced that we will be 'rolling' on a PvP server called Sienna Canyon. I select that name and click to proceed.

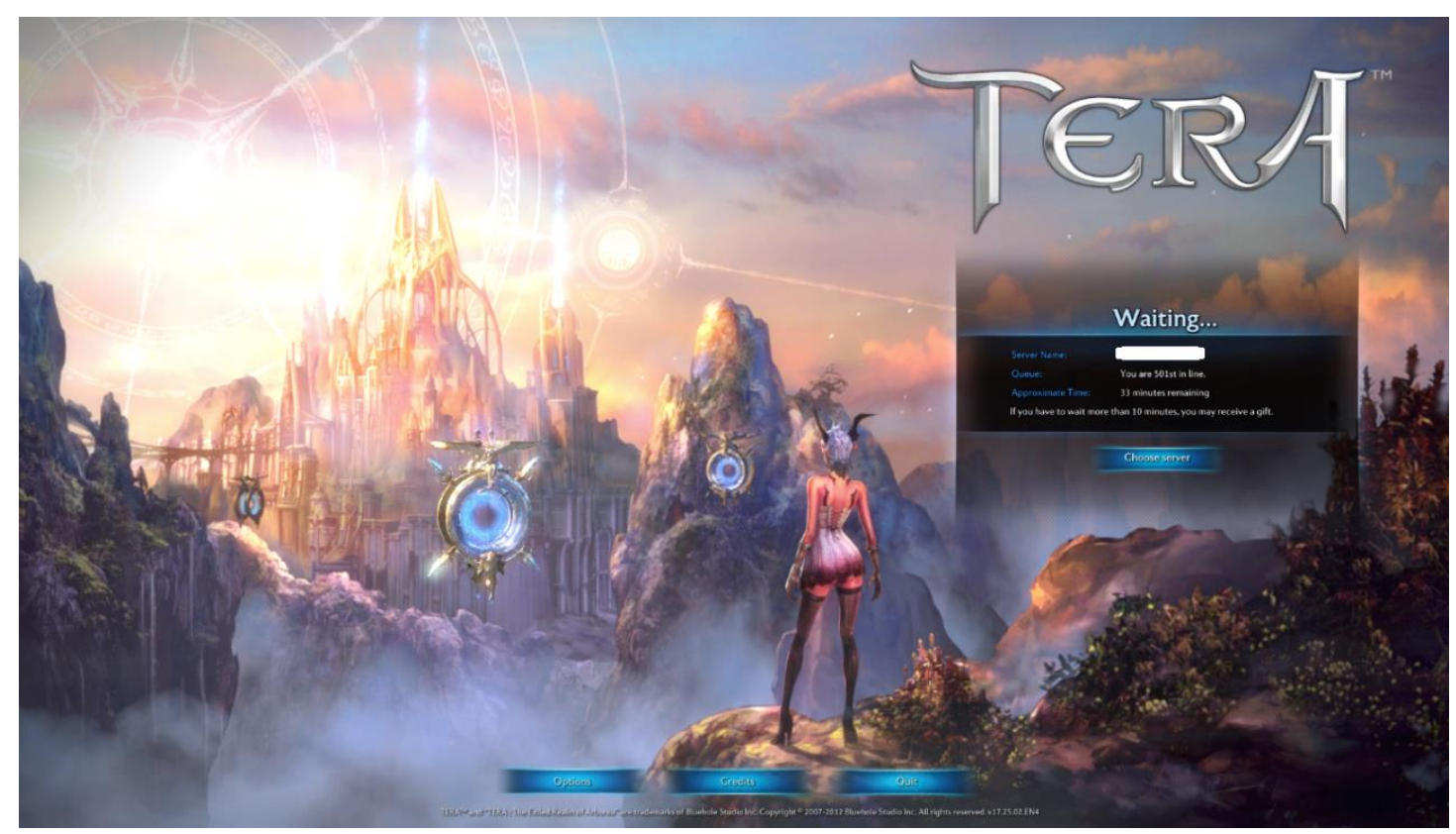


On the next screen I have the option of creating a character. From reading information on the game's website I have already decided what kind of character I want. The first choice is race: I am presented with an array of strange not-quite-humans, big and small. There are the amani, large and fierce-looking with scaly skin. There are the castanics, devil-like and sexy with both genders showing brazen skin. There are even the popori, short, round and fur-covered with fluffy animal faces. What I want is a high elf, a pale, willowy, pointedeared humanoid. I choose female for my gender (she poses coyly) and move on to the next step: class.

There are eight classes to choose from, representing different combat styles. Clicking on each shows an example of an elf of that class, armed and armoured and dramatically posed, although many of the women's tilted hips and pouts don't seem very practical. There are short blurbs describing each class's specialties and a difficulty rating, with five stars indicating the most complex to play. I choose a priest, who appears wielding a staff and wearing what looks like an ornate cocktail dress. Priests are healers in TERA, and healers (my past gaming experience tells me) are usually in high demand; I'm hoping this choice will make it easier for me to find a place in groups. High elves get bonuses to do with magic, which is also what priests use, so the race choice was strategic as well as aesthetic.

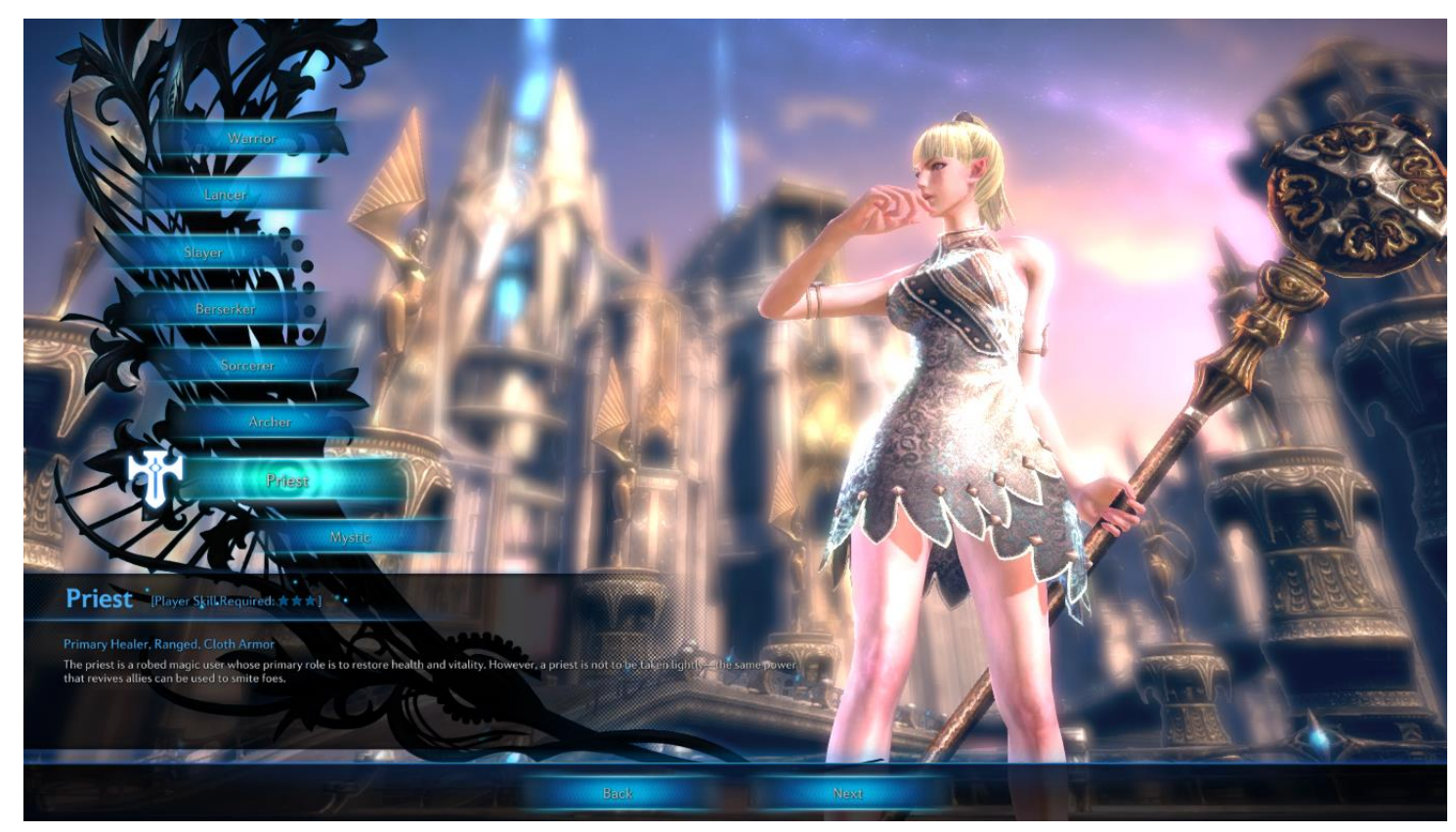


The next step is to customise her appearance, and it is a very involved process. Hair style and colour are relatively simple. The face is more complicated. Although I know I will be looking at the back of her stylishly coiffed head most of the time, I can tweak my elf's facial features down to minute details. 'Make-up' changes her appearance at least as much as changing her bone structure does! I can even modify the degree to which her mouth hangs suggestively open with a slider labelled 'gape' - just one of many factors in the extreme sexualisation of female characters in this particular game. Whatever I do, my elf looks a little vapid. This seems to be a racial feature, and unavoidable.

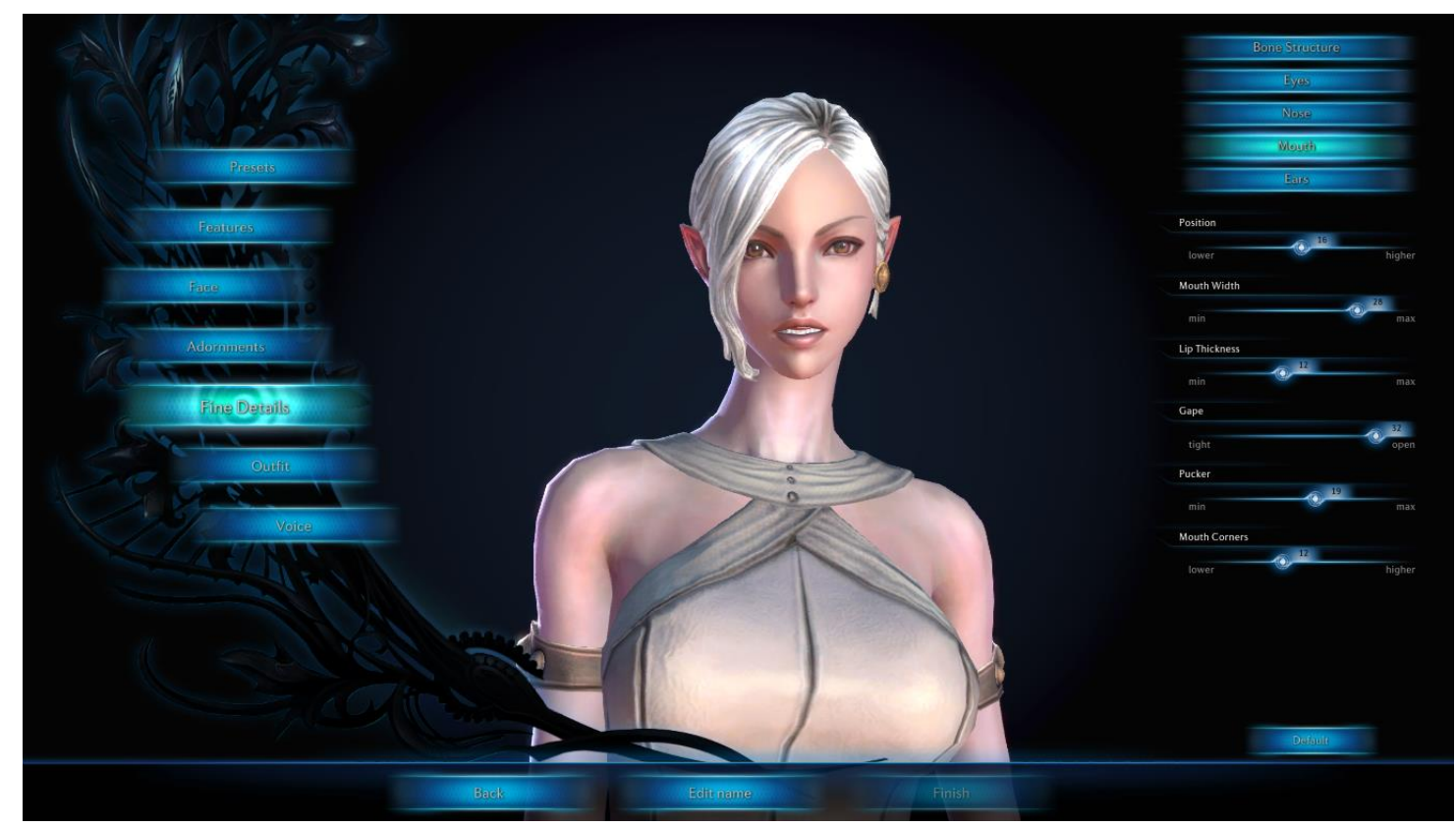

The last thing is to name her. I type in my chosen moniker and click to finish with fingers crossed. Success! Sadly I can't play my new elven priest in the actual game world just yet. In just a few days we pre-purchasers will get an early look in before open beta. For now, I do as my guildmates are doing: take screenshots to show off and comment on other people's character designs. On our guild's website, members are changing their usernames to reflect the characters we will know them by from now on. Everyone is itching to get into the game and start playing for real. 


\section{Introduction}

"TERA is an MMO that keeps you on the edge of your seat. This is not one of those cast a spell, sit back and relax MMOs. This is a visually breathtaking game that's rich in story and physically engaging. In TERA, you're in control. YOU aim, YOU dodge, YOU move, or it doesn't happen. It's like an action console game and a traditional MMO got together and produced an heir to the throne of the video game kingdom."

(Promotional description from http://www.enmasse.com/products/tera)

Massively multi-player online (MMO) games are increasingly a major commercial industry, grossing \$13 billion internationally in 2012 (Newzoo 2012) with new titles regularly developed on hundred million dollar budgets. As these games have grown in popularity, players have emerged who are dedicated not to one specific game so much as to the genre, the idea of online gaming; news websites such as massively.joystiq.com and mmorpg.com follow rumours, releases and reviews of a large number of games at any given time. Such players are likely to try out any new offering, whether for free during limited testing events or by buying an account. Of course, with intense competition not all games can be successful in the long term, and many release to a burst of enthusiasm followed by an exodus of players who quickly lose interest. This cycle - of players entering a new game world, participating in the game, developing characters, and then abandoning them in favour of a new world to dabble in - has been less studied than the more stable (relatively speaking) communities of large, well-established online games. As more such games become available every year, the fickle migration from game to game has developed into a culture of note in itself, as conventions are carried between individual sites with the 'churn' of people coming and going.

In the following chapters, I will explore how players of the online game 'TERA' related to each other and to the game's developers during TERA's launch and volatile first few months. Asking how players organised and identified themselves in a brand new virtual environment, I investigate their expectations, assumptions and emergent patterns of behaviour. As knowledgeable, discerning customers, these 'gamers' had a complex relationship with the companies that produced the games they played. In 
order to participate in TERA and its social world, players had to accept the rules and terms of the game, including constraints which were built into the code which defined the virtual world. Players did not have access to this software code; developers did, and could change it, but were not inhabitants of that world themselves. This left players building their own societies in a world that was controlled and constructed - but not by them. Nonetheless, players exerted significant influence over TERA during my research. I will argue that although as players they submitted to rules written by others in order to participate in the game, they used their role as customers paying for a service to influence developers' decisions as much as game design subtly influenced their own. Empowered by their sense of consumer agency, they critically evaluated the game as a service and demanded that developers work for them as part of the commercial relationship they had entered into.

\section{Play, Games and the Internet}

"Despite frequent public dismissals and indictments, videogames do constitute a complex and nuanced set of multi-modal social and communicative practices, tied to particular communities and consequential for membership and identity" (Steinkuehler 2004:522). Such dismissals often come in phrases like 'it's just a game', but given the integral role that play has in human cultures - not to mention the complexity of virtual worlds which may go far beyond the normal concept of a game - there is little reason to say 'just'. Indeed, anthropologists have long made it their business to investigate phenomena which might otherwise be dismissed as 'just' parts of everyday life. Johan Huizinga argued that play is partly defined by becoming so absorbed with the pretence of one's game that one forgets - "temporarily at least"

- that it is 'just' pretend (Huizinga 1950:8). Like many of the most powerful forms of human cultural experience, "play allows us to be totally frivolous about important things in our work-centred lives or else completely serious about things that are trivial" (Gruneau 1980:68). It would be short-sighted to dismiss play behaviour as inconsequential simply because it is seen as non-productive, especially at a time when digital games are beginning to bring adult play out of the shadows of illegitimacy. 
Massively multi-player online games are significant for a number of reasons, not least of which is the sheer number of people playing them: market research company Newzoo reported 400 million players in 2012 (Newzoo 2012). They are also, in many ways, more than simply games. With persistent virtual worlds, games like TERA provide a sense of 'place' with room for serendipitous encounters, a 'third place' (Steinkuehler and Williams 2006) external to any individual player. Persistence also allows players to build lasting groups, identities and relationships which would not be possible in temporary play spaces. When thousands of people begin to work together (or against each other) in a specific environment, and to develop norms, values and distinctions within that environment, social scientists should take notice. Although some are wary of any relationship with no face-to-face element, ${ }^{1}$ players certainly treat game worlds seriously. Whereas most games have clearly defined boundaries in both space and duration outside of which the conflicts of the game are left behind (Henricks 2008:177, Huizinga 1950:10), an online world is a 'playground' which persists and continues to develop between play sessions. There is no opportunity for a friendly handshake at the end of play.

The scope of this thesis does not allow for a full history of online games and virtual worlds, but a brief outline will help to illuminate the heritage of the massively multiplayer genre to which TERA belongs. The first multi-player online game world, a text-based fantasy game called MUD1, was available from 1980 and began a whole genre of MUDs or 'multi-user dungeons'. These typically drew heavily on the fantasy adventure tradition of Dungeons and Dragons (a pen-and-paper role-playing game), as online games still do (Taylor 2006b:22). Achterbosch et al. (2008) and Bainbridge (2010) provide excellent summaries of the development of online games; here I will only point out that they progressed from solely text-based (e.g. LambdaMOO [Dibbell 1998], The Palace [Suler 1996]) to graphical representations (e.g. EverQuest [Taylor 2006b], Second Life [Boellstorff 2008]). This generally made them more accessible and easier to navigate, but came at the cost of player creativity, for where any player could quite easily add or modify written descriptions, in a graphical world players were limited to manipulating elements that designers provided for them. Player embodiment is much more restricted, for instance, when one must create an

\footnotetext{
${ }^{1}$ For example Sanders et al. (2011), who in their concern over internet predators treat online friendship as if it were dangerous in itself.
} 
avatar out of pre-programmed pieces rather than through free description (Taylor 2003).

Over time game-based worlds have also generally become more scripted, tending towards what gamers call 'theme park' games - those which lead players from programmed activity to programmed activity - as opposed to 'sandbox' games, which rely on players themselves to direct gameplay and build features. This distinction bears some resemblance to Caillois's (1961) continuum between ludus (structured, competitive play by rules) and paidia (free, spontaneous, aimless play). TERA was essentially a theme park game, although its political system was a limited sandbox within which player interaction could affect the world. Although there was a broad diversity of massively multi-player games available at the time of this research, the typical 'MMO' was of a type modelled on World of Warcraft (very much following in the Dungeons and Dragons tradition), and TERA tended toward these conventions. More social, less game-like worlds like Second Life (Boellstorff 2008, Malaby 2009b) tended more towards the creative, sandbox style.

\section{About TERA}

TERA was an online game produced by En Masse Entertainment which at the time of this research had just launched for a North American (and thus wider Englishspeaking) market. It was a game of the type known as 'MMORPGs'; the acronym stands for 'massively-multi-player online role-playing game', and it is a term best defined by dissection.

'Massively multi-player' signifies a game world in which large numbers of players participate at once alongside one another. Whereas 'multi-player' can signify as few as two people sharing a play environment, co-operatively or competitively, the 'massive' in MMORPG reflects the fact that hundreds or thousands of players can be present in a world at once. Unlike a game environment created temporarily for specific friends to play in, a persistent virtual world is independent of any specific connection and continues to change and be changed after any individual player logs out (Boellstorff 2008:45). Additionally, "persistence is critical for the social dimension of every virtual world since it...provides a sense of linear progression and 
stability, upon which participants can draw future trajectories" (Papargyris and Poulymenakou 2009:4), supporting the development of a game culture.

'Online', naturally, means that the game is accessed through the internet. Users download a game client which is installed on their computer. The client requires communication with the game's servers in order to retrieve information and keep the user's computer up to date; when other users act, or the game responds to user actions, all users who are 'present' have to be updated in real time. This means that without an internet connection and authorised access to game servers, the client itself is useless. For TERA and most contemporary MMORPGs, access required a one-off payment for an account plus subsequent payments of a monthly subscription fee, a model which will be discussed further in chapter six.

The term 'role-playing game' in this context refers to specific game elements. The majority of TERA players did not role-play in the sense of creating personae and acting or speaking in character. There was a dedicated role-play server for those who did play this way, but outside of that server it was rarely discussed. TERA was a roleplaying game in the sense that each player had one or more characters that they controlled and developed as they progressed through the game. Each character gained levels (a general indication of strength and experience), new equipment, and abilities as they advanced, becoming more powerful and generally more complex to play over time. This character development followed very much in the style of older, non-computerised role-playing games like Dungeons and Dragons, with designated combat roles (as healer, attacker, etc.) determining what which attributes it was desirable to increase. Characters required a lot of effort to develop, but players I spoke with did not imbue them with personalities of their own.

Technically, then, TERA was an internet-based game with both co-operative and competitive elements, played within a virtual world in which players were represented by customisable characters. Players could form groups of varying degrees of persistence, and pursue objectives provided by the game (although there was no way to definitively 'win'). In 'lore' - the gamer term for in-game mythology and background stories - the world of TERA was a dream. The two major continents, Shara and Arun, were the bodies of two primordial titans, and they dreamed the 
game world and its inhabitants into being. This premise led to surreal landscapes in bright colours, full of cascading blossoms and lush forests, elaborately fantastical cities and dark gothic manors. By contrast, the ultimate enemies in the game (called 'argons') were everything the dream-world was not: cold, metallic and homogenising. Light or dark, the world of TERA was beautifully detailed, as well as full of little comic touches that helped to bring it to life. The exceptional level of detail in character design, outfits and animations was something often commented upon and praised by players as they explored.

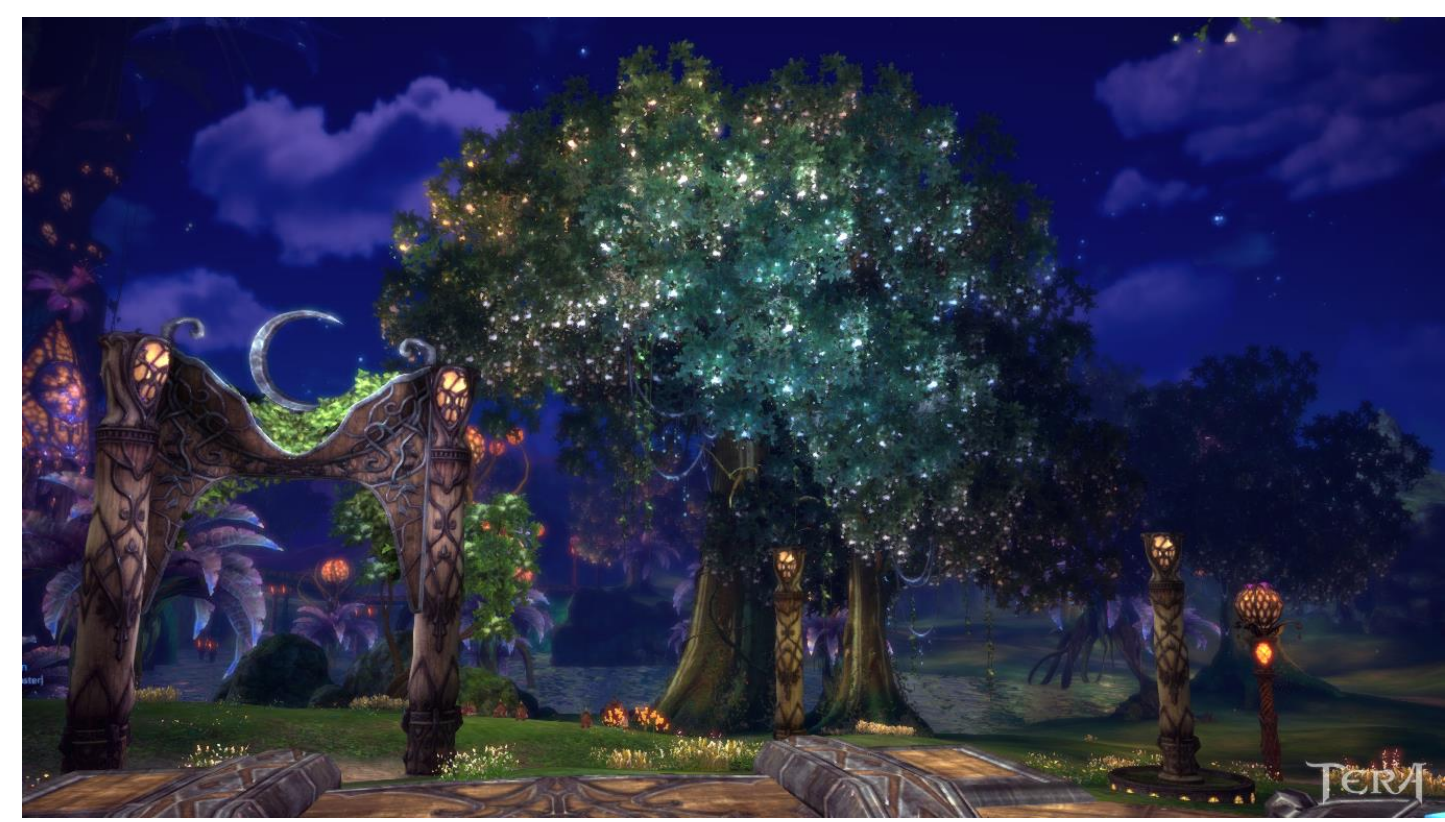

A magical forest bedecked with glowing lights (personal screenshot).

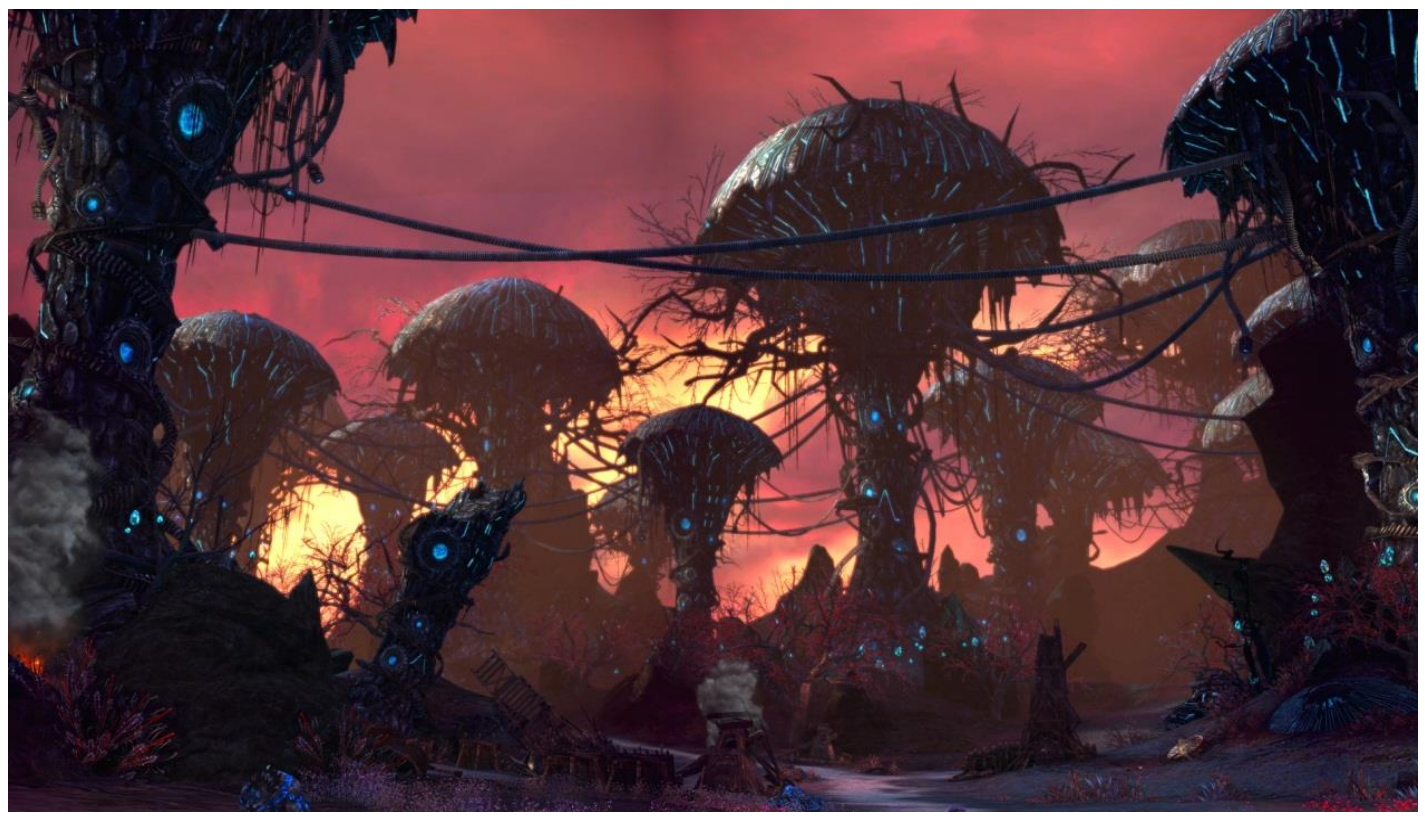

An argon-controlled landscape (image from tera.enmasse.com). 


\section{Structure, Agency and Practice}

The time during which I played TERA was a volatile one for the fledgling game community, with large numbers of people joining the game and large numbers leaving, game systems being tested and improved, and hierarchies established and disputed. Because of this, processes of change - social, structural and technical quickly came to the fore in the research. Online games are always subject to change through regular updates, and should be considered as open-ended and never truly finished (cf. Stenros and Sotamaa 2009). When players criticised aspects of the game, they almost always added their thoughts on when or how the developers might fix current issues. TERA was not a finished product that players had to accept or reject as it was at release - instead, it continued to evolve in response to the interplay of player desires and design necessities, taking into account emergent patterns and meanings. Players were enmeshed in a continuing relationship with the creators of the game (Stenros and Sotamaa 2009:4), and the world and its rules were responsive to user practices.

These observations touch on an important tension present in any culture: that between structure and agency. 'Structure' is "a kind of founding or epistemic metaphor of social scientific - and scientific - discourse" (Sewell 1992:2), and Fekete (1984) calls it "the structural allegory"; it suggests an underlying pattern or logic from which social phenomena arise. The concept has outlived the school of 'structuralism' as exemplified in anthropology by Lévi-Strauss, but it retains a heritage from structural linguistics, notably Saussure's opposition of langue (language) and parole (speech). Where parole is language as put into practice by real people in specific acts or 'events', langue is an abstraction which does not exist in its entirety in the mind of any individual person. Rather, it belongs to speakers' 'collective consciousness' (Sturrock 1986). Structure is to observable society as 'language' is to speech acts: it guides and limits what people do, making them intelligible to others in the process; it is "both enabling and constraining" (Giddens 1984:162). Thinking in terms of structures always means thinking in terms of structural relations, where concepts are connected to each other in a decentred way and a shift in any part affects all others related to it. This relational thinking is important to the holistic perspective of anthropology. 
The tension, then, is between the relations which structure acceptable action within a system (a culture) and tend to lead to their own reproduction, and the fact that individual human agents are not drones but rather have the ability to choose how they act. Games are actually well-suited for exploring this tension because play, within often arbitrary rules, "combines in an experiential unity both social constraints and spontaneous behaviour" (Csikszentmihalyi and Bennett 1971:57). In his account of Hawaiian history Sahlins (1981) gives examples of agents using the cultural structures at their disposal to pursue their own interests, and transforming the nature of the structures themselves in the process. Many scholars have warned against the fallacy of reifying structures and treating them as if they had a tangible existence, but virtual worlds provide unique circumstances for the concept of structure. In virtual worlds there is a real structure, external to the people who participate in the world: the software. The programming of a game like TERA connects the virtual environment, forms of embodiment, and certain institutions (such a 'guilds') to each other as part of a whole reality. Players cannot choose to disobey that which is part of the fabric of the world, unless they choose to not participate in the game world at all. Online games thus provide a wholly different kind of context for communities and cultures to develop in, one where rules written by one group of human beings operate like the laws of physics for another group, the game’s players.

The result is an ambiguous kind of structure, where the laws of physics and cultural 'rules' are not as distinct as we usually assume them to be. In our physical world, the former may be manipulated to some extent but not outright changed, while the latter are influential, but their application is ultimately in the hands of living people. Advances in technology that blur this distinction by allowing humans to manipulate things once firmly in the 'nature' category (what Rabinow [1999] has dubbed 'biosociality') actually bring the real world closer to the virtual case. In TERA, the structure of the game constrained everything that players might want to do, including self-representation (in the form of characters). Ortner (2006) argues that agency and power are not the same thing, but that they are linked: the powerful have more agency, because there are fewer limitations on what they can actually do. The less powerful can only exercise agency in certain areas where nothing prevents them from acting as they choose. Within a game world players are certainly less powerful 
than developers, who have access to the software that constitutes everything in that world.

As quite literal intelligent designers game developers had god-like control over their own worlds, but they were a strange kind of gods - ones who were accountable to the people they created for, and often capitulated to their demands. En Masse Entertainment was a company and maintained TERA in order to make a profit from it, which made them a service provider and TERA players their customers. As Giddens says, "all forms of dependence offer some resources whereby those who are subordinate can influence the activities of their superiors" (1984:16); for players, their ability to choose where they spent their money was such a resource. This meant that both the environment and the terms of the game itself were responsive to player criticism - something which cannot be said of physical laws in the 'real' world. All aspects of TERA were constructed, with human minds behind the design, so the process of practice feeding back into structure was tied up with the marketing process of 're-qualifying' a product to suit the changing desires of customers (Foster 2007:715). In the following chapters I wish to explore how player agency operated within a quite 'real' structure (as real as the game world), controlled by conscious human design and commercial interests.

\section{In This Thesis}

In the following chapter I will briefly discuss the ethnographic methods that I used in my study of TERA, including how they contribute to an insightful analysis of the site and the challenges that site raised. Contributing to the on-going discussion of ethics in online research and in participant observation more generally, I describe the special ethical issues raised by this situation and how I dealt with them. Chapter three provides a more thorough introduction to TERA by outlining the history of the game during the period of my fieldwork, which encompassed a startling amount of change in just a few months. The frequent movement of players between similar games emphasised how competitive the MMORPG market was, and how TERA was connected to other games through the movement of people. 
Chapter four parallels chapter three in describing a life cycle, this time of the 'guild' (an important form of player group) that I joined. The conflicts and problems of Bellicus, whom I observed more intimately than any other group of TERA players, reflected emergent issues in the wider game as they appeared on a micro-social scale. Players tended to treat TERA itself as a kind of marketplace, where they shopped around for the guild or friendship best suited to their immediate needs. Supported by internet technologies, they could impulsively abandon and replace social connections in a similar way to how they moved between games.

In chapter five I explore the ambiguity around the idea of a 'fair' or 'right' way to play. In general players expected equality of opportunity, idealising TERA as a meritocracy based on individual skill - an ideal linked to the capitalist culture in which I argue TERA players seemed to be firmly entrenched. How well the ideal was actually achieved was always debated, with players looking to En Masse to ensure that balanced reigned. Inequalities were accepted as part of the game, but players wanted a kind of egalitarian base line from which to compete. This created a kind of idealised capitalism, in which work yielded reliable rewards and all participants were guaranteed the same treatment from the system.

The relationship between game developers and players is the focus of chapter six. They operated as two distinct factions aligning roughly with 'structure' and 'agency' as concepts, in that the developers were an authority separate to rather than part of the player base. Developers could freely impose rules and conditions on the world of TERA, but players were not obliged to continue playing. A balance emerged which was founded on the commercial nature of the relationship, with customers boldly pushing to be heard and listened to if En Masse Entertainment wanted their money. Players took an active role which suggested an interpretation of their consumer role as entitled and empowered. In the concluding chapter, I will discuss how an understanding of TERA's players can inform thinking about contemporary society more generally. They displayed a particularly informed and empowered consumer identity, which did not deny the virtue of consumption but instead embraced it as the basis for particular rights. For these players technology was both enabling and constraining, but where they lacked the access or technical expertise necessary to act, 
they applied pressure to developers and often succeeded in having the changes they desired made for them.

An appendix at the end of this thesis contains a glossary of terms and abbreviations for easy reference. Sections in the text are prefaced with italicised quotes which come from research participants unless otherwise stated. These quotes are intended to illustrate the issues under discussion in players' own terms, and are deliberately provided with minimal attribution to reflect their general relevance. 


\section{Methods and Ethics}

Virtual worlds can seem like an entirely new and different mode of being, but in fact a site like TERA often does not require any more modification of our methods than the specific conditions of any site. There is now a substantial literature supporting online ethnographic research, notably the recent Ethnography and Virtual Worlds: A Handbook of Method (Boellstorff et al. 2012) - but it remains a relatively new field, in which conventions are still being established. To contribute to the on-going discussion of online research methods, as well as for transparency, in this chapter I will discuss the methodological decisions I made for this study of TERA. I will focus particularly on methodological and ethical factors which are unique to virtual world and online game research.

\section{The Researcher}

"An anthropological fact only becomes so once it has been recorded: it is an account by an observer of something" (Sturrock 1986:40). The instrument of data collection in participant observation is the researcher - she who sees, hears, feels and interprets experiences in the field before subjecting them to analysis. As such, it is useful to know a little about the person behind the interpretations. I was 23 years old at the time of this research, and had been using the internet regularly (and chiefly for social purposes) since the age of ten. I was also exposed to video games from a young age, and had been playing them extensively for about seven years, with a preference for the role-playing genre. As such, sitting at a computer for hours at a time was not an especially novel experience for me (unlike some internet researchers, e.g. Markham 1998), and online game interfaces were relatively easy for me to understand. I played the online game Guild Wars for some years, something I mentioned when asked about previous gaming experience in my guild application. ${ }^{2}$

\footnotetext{
2 Those familiar with Guild Wars will know that it includes many of the conventions and mechanics of other MMORPGs, but is not entirely the same kind of game as more prominent titles such as EverQuest, Lineage, and World of Warcraft, from which TERA takes its lead.
} 
For about three years online games had been a particular personal and scholarly interest of mine. In short, I had enough prior knowledge to decode cryptic abbreviated statements such as "lfm nt 2/5" when they appeared in-game.

The fact that I self-identify as a 'gamer' certainly coloured this research. With video games so often the subject of moral panic in popular media, there is a temptation to counter this negative press with a more positive account. The truth of the matter is, of course, that it is more complicated than that; reports of widespread sexism and harassment in online gaming (e.g. Fletcher 2012) are genuine and concerning. TERA's character models were frequently hyper-sexualised and could be interpreted as misogynistic, and public chat in the game was often rife with sexual references and threats which some found distasteful. I do not wish to ignore these issues, but they are beyond the scope of this thesis and require more thorough research to deal with them fairly. Other negative stereotypes of gamers - as lonely, anti-social, unproductive young people - are challenged by the data presented here.

My relationships with other players in TERA were a mixture of familiarity and difference, in that I was meeting other gamers and comparing play experiences but also being exposed to forms of gaming I would not normally participate in, such a PvP ('player versus player') combat. My gaming background enabled me to collect useful data within only four months; without some practical experience, it would have taken much longer for me to become competent with the game and to establish rapport with my experienced participants. At the same time, my familiarity made me less likely to question basic features and conventions present in TERA, and I tended to assume that I understood the meaning of terms I had seen and used before in other games rather than interrogating their meaning in TERA specifically. Acting on the assumption that I knew what I was doing probably helped in securing rapport with players, but it may have led me to overlook some things.

The fact that I was a female player was surprisingly unimportant in my experience. Female characters were commonly controlled by male players, and people I spoke with in passing often seemed to assume that I was male until told otherwise. My guildmates knew that I was female, but the guild included many other female players and couples who played together, so this was not especially anomalous. In voice chat 
(where gender was most obvious) my New Zealand accent was far more interesting to my American and Canadian guildmates than was my gender. This may well have been different in another guild.

\section{Methodological Decisions}

In selecting a site I chose to study a new game from its launch rather than an existing virtual world in order to observe how people settled into a new game community. This allowed me to watch how groups of players (who were generally competent gamers already) interacted with the specific affordances and demands of a new environment, and how local norms emerged within the conventions of the MMORPG genre. This contrasts with the majority of previous online game studies, which have focused (quite reasonably) on games with established populations. With time, I came to see the flow of people from one game to another as a significant phenomenon in itself, as discussed in chapter three. TERA was a convenient upcoming release at the time of planning. It provided a fan community that was already in communication with each other in anticipation of the game, but did not yet have access to the game world and its particular structuring elements. I approached the TERA community after most fans had participated in closed beta testing (see chapter three), which meant they started with a greater understanding of TERA's gameplay than I had; nonetheless I joined a guild and became part of a fledgling community of soon-to-beplayers before TERA was officially released.

Joining a guild was a major methodological decision. Guilds in TERA were persistent groups of up to 300 characters (some of whom might be controlled by the same player) which were formally recognised in the game. Guild names were displayed alongside character names, and members could see a list of who was online in their guild at any time as well as chat in a separate guild-only channel. A substantial amount of previous research into online games has focused on guilds (see for example Ducheneaut et al. 2006, Jakobsson and Taylor 2003, Johnson et al. 2009, Nardi 2010, Williams et al. 2006); not only are they the most important social group or faction in most MMORPGs, they also provide an ethnographer with a more reasonably limited field of potential informants. Once accepted into a guild I was able to use its website to introduce myself, explain my research, and give other members a 
chance to ask questions or to opt out in a forum where my posts would be seen. I chose to be entirely open and honest about my intentions and practices as a researcher, although as anthropologist Bonnie Nardi observes of her own gamer participants, my guildmates were generally "incurious about my research" (2010:31). I was able to develop rapport with players within the guild much more easily, through regular contact, than I could with other players; over the time that I played I got to know several prominent members of my guild quite well.

'Bellicus', the guild that I joined, advertised themselves as a compromise: the ambition and goal focus of a 'hardcore' guild without the associated pressure to play frequently for long periods of time. This suited my anticipated level of competence: I had neither the available time nor the skill to keep up with a dedicated 'hardcore' group of players. Bellicus' leaders were highly organised and invested in building a strong guild for this new game, making them a promising group for studying how TERA's community was established. I make no claim that Bellicus was representative of a 'typical' guild, but they were one of many groups which had already formed before I approached them. The guild is discussed in greater detail in chapter four.

Joining a guild before TERA's release determined my choice of 'server', out of the eleven versions of the game world in which I could choose to play. Each server's world was nearly identical, but fell into one of three categories with slightly different rules. My server, here called Sienna Canyon, was a 'PvP' or 'player versus player' server, meaning that players were allowed to attack other players without warning in the open areas of the world. This encouraged competitive play and greater ambiguity about what constituted fair play, as will be discussed in chapter five. The other server types were 'PvE' ('player versus environment') - which did not allow players to fight each other without mutual consent - and a single 'RP' or 'role-play' server, which operated as a PvE server with some extra rules of conduct. These categories were common across online games, and many generalisations in this thesis relate to $\mathrm{PvP}$ servers specifically.

The last major decision that I had to make before I was able to begin my research within the world of TERA itself concerned the character I would choose to represent myself. I use the word 'character' in place of the more widespread 'avatar' because of 
the distinction that Bainbridge highlights (2010: 56), namely that 'avatar' tends to refer to singular self-representations and is more common in non-game virtual worlds, whereas 'characters' are often multiple and are less intimately representative of their controllers. Taylor (2006b:14) has insightfully discussed how her EverQuest character's race and class affected her role and access as a researcher. Bearing her account in mind, I chose the priest class based on the assumption (formed from playing other online games) that healers would be relatively scarce and thus in high demand, giving me easy access to group play. The priest was a straightforward support class which was useful in most situations, and had a clear role in groups which proved relatively simple to learn.

Throughout the planning and field periods of this research, I have kept in mind an underlying commitment to studying the game community as it manifested on the internet, without including the ways that TERA fit into players' wider lives. This was simply a practical limitation, based on time, resources and the scope of the project. I do not deny that "observing online phenomena in isolation discounts social processes offline which contribute to an understanding of use of the internet as a meaningful thing to do" (Hine 2000:27); nothing online is wholly isolated from the real world, even where escapist fantasy is intended. However, while others have specifically studied how internet use fits into users' wider lives (Miller and Slater 2000, Turkle 1995), my research interest was in the communities and norms which formed within the games, and that is where I have focused. In this regard, being only a part-time resident in the field - with all the associated risks of missing important developments - was part of participating on the same level as players, a feature of the method rather than a problem with it (Hine 2000:21, O'Reilly 2009:216).

\section{Ethnographic Research}

While there have been a number of fruitful studies analysing social life in online games using quantitative methods (e.g. Ang and Zaphiris 2010; Debeauvais et al. 2011; Ducheneaut et al. 2006; Seay et al. 2004; Snodgrass et al. 2012; Williams et al. 2006; Yee 2006b), the contributions of this thesis play to the strengths of an anthropological approach by employing ethnographic research methods. This approach has been very productively applied to other virtual worlds and online 
games (see for example Boellstorff 2008, Markham 1998, Nardi 2010, Pearce 2009, Taylor 2006b), where immersion in the technical as well as the social environment has led to important insights. I approached this research with an open-ended interest in social life and conduct within MMORPGs. Throughout the research the changing conditions of the game itself guided my focus, leading to topics I could not have predicted beforehand.

The core of my research in TERA was participant observation. Online games and virtual worlds provide some unique opportunities for this method, and for ethnographic research in general. For example, my entry into the field of TERA as described above was essentially identical to the ways that my guildmates first entered the game world. One of the barriers to true immersion in traditional ethnography has always been that an anthropologist cannot know the experience of being born and raised in a culture other than her native one. By contrast, all online communities are inhabited only part-time by their members, who come to them from different backgrounds based on their face-to-face lives and locations. Users necessarily endure a period of being a 'newbie' when they enter any new site. Other researchers (Boellstorff et al. 2012, Steinkuehler 2004, Suler 1996) have highlighted the usefulness of newbie status when making mistakes or asking potentially obvious questions; it is normal for "gamers who have already mastered the social and material practices requisite to game play [to] enculturate, through scaffolded and supported interactions, newer gamers who lack such knowledge and skill” (Steinkuehler 2004:525). This helps to make the ethnographer a less remarkable figure than she might be in a geographical, face-to-face community. Like ethnography itself, playing TERA was “a process of learning” for everyone (Stewart 1998:21).

Participant observation is in some ways a natural technique for studying online game worlds because, as Nardi says, "it would be impossible to penetrate the game without becoming engaged as a player" (2010:28). Quite literally, presence in the game world of TERA required the creation of a character. This character needed a physical appearance and a name but also a class, defining a combat role, before it could exist in co-presence with other players. To access more than the limited beginner's area of the game I had to 'level up', which required fighting monsters. It was thus necessary 
to participate in gameplay just to get around; there was little to do in the game world but play the game. Joining a guild facilitated this as well as access to more players, but it also situated me in the game community, giving me allies, enemies, mentors, superiors and (eventually) inferiors - much as an anthropologist who is given a kinship position (cf. Briggs 1970) becomes situated and enmeshed in specific relationships. When conflict arose in my guild (as recounted in chapter four) I was caught between two factions along with my fellow members, forced to decide methodologically but also personally which leader I would follow. I played less intensively than my guildmates did early on and levelled more slowly than most, and so was only occasionally able to join them in the game's level-based challenges. Despite this I was present and involved in daily guild life, participating in guild chatter, posting on forums, taking part in events that the leaders organised, and asking for or offering help.

Where the ethnographic research of anthropology has traditionally involved travel, online research can be conducted from home, in my case from the same computer where I surf the web, write, and play other games for leisure. For ethnographers who do not travel to a distinctly different physical place, "the movement is a cognitive one” (Rapport 2000:72). The fact that TERA was a subscription-based game that I would not normally have played helped to maintain this cognitive distance, and I frequently recorded images and text to assist in writing up field notes at the end of each play session. Like Suler (1996), I preferred to write field notes reflecting my experience and to use the detailed chat records which online research allows as supplementary rather than to rely on them alone. As well as other players' words, I recorded my own reactions: the thrill of my first successful player kill (in selfdefence!); the discomfort of accepting a former enemy who had defected to our guild. These experiences helped me to gain insight into how and why people played TERA.

My period of field research lasted for approximately four months, from the open beta weekend starting on April $19^{\text {th }}$ to a gradual withdrawal from the game itself in August. On average I played four days a week, in sessions of two to five hours. For about a week before this period I was in contact with my future guild, explaining my research and introducing myself among other new member introductions; until early September I maintained a subscription to TERA, logging in occasionally for short 
visits during the last month. Throughout the research I closely followed my guild's website and forum discussions and more casually read TERA's official forums, although no systematic analysis of these was conducted. TERA's official release date in North America was May $1^{\text {st }}$ 2012, so the period during which I played encapsulated the game's first four months, a critical time for a new game to establish a player base. As will become evident in the following chapters, this unstable period in the life-cycle of a new game is under-researched but important for the many online game players who move regularly between games, rather than remaining entrenched in a large and successful game like World of Warcraft.

\section{Ethical Considerations}

I began this project with a strong ethical and methodological stance: I would treat whatever personae players presented online as the subjects of my research. I would not ask for personal information pertaining to my participants' 'real lives' or attempt to verify any such information they offered me. Personal information was extremely difficult to verify without meeting participants in person (which was outside the scope of this project), but it was also unnecessary to "an ethnography that treats cyberspace as the ethnographic reality" (Ducheneaut et al. 2010:137). Although some scholars of online communities have restricted their participants to those they could meet in person (Turkle 1995) and some have used face-to-face encounters to supplement virtual world data (Taylor 2006, Pearce 2009), these were methodological decisions, related to the particular research questions under consideration (Taylor 1999). Because I was interested in the in-game community itself rather than how the game fit into players' real lives, I resolved to work with players as I knew them within the game. As well as being ethically simpler and sidestepping the issue of validity when dealing with people solely online, this choice reflected a commitment to studying online games "in their own terms" (Boellstorff 2008) and taking them seriously as a site for the development of culture.

In fact, this initial stance was undermined by the behaviour of the players themselves. Unlike participants in more social-focused virtual worlds like Second Life, TERA players on my server did not seem interested in role-playing or presenting themselves differently. Although 'real life' information was rarely shared 
in sever-wide chat (and my sense is that what was shared there would be unreliable), my guildmates shared information among themselves very freely. Self-introduction posts on the guild's website often included gender, age, occupation, details of the player's family and sometimes an uploaded photograph - all without special prompting. Guild members seemed to want to get to know each other as people who were going to be working together in the game rather than as anonymous characters in a virtual world. This relative transparency was reinforced by the common use of an external voice chat program, which made details such as gender more difficult to obscure. Most guild members I knew were not at all reticent in sharing basic personal information, and at one stage an 'Overshare Thread' was created in which members shared photographs of their young children or, in one case, pets.

From this behaviour and voice conversations with my new guildmates (especially the 'icebreaker' activity described in chapter four) I quickly deduced that Bellicus' membership did not, by and large, see their game characters as an opportunity to be someone they were not. When men played female characters, they commented on and joked about that choice without suggesting any identity play. Players viewed their characters as instrumental, as vehicles through which they acted in the game world, but they interacted with other players as people and not as characters. This was particularly notable in voice chat, for those who used it; with the occasional audible intrusions from pets or children needing attention, there was very little mystery maintained between players. Despite this surprising attitude on the part of my participants, I maintained my policy of not soliciting any information unrelated to the game. Real life information that was shared did allow me to come to know my guildmates better, but I have declined to analyse or publish details of such information both out of respect and in the interests of methodological consistency.

Ethically, it was easier to be answerable to the guild than it would have been to secure consent forms from players I might encounter only briefly in the course of play. Guildmates had access to statements of my research methods and intentions, and I made a point of mentioning my thesis in conversation occasionally to remind them of my role as researcher as well as fellow player. This could be done in the course of normal conversation, and often humorously, as in one memorable example: 
Barsabba: lol 1 more war on bunny girls [a guild]

Turrek: Sluts

Kenton: sluts? where?

Meruma: loll

Jelena: thatway

Jelena: I mean

Meruma: LOL

Jelena: no

Turrek: haha

Kenton: jelena o_o

Taydis: lul

Jelena: that way

Elizabeth: Sorry Jelena but... going in my notes :P lol

Such reminders embraced the principle that "the informed consent process is necessarily dynamic, continuous and reflexive" (AAA 2012, section 3) and should be available for negotiation. As well as endeavouring to make myself known as a researcher, including to members who joined after I did, I used electronic consent forms (hosted on Google Docs so that participants were not required to provide an email address if they did not wish to), into which participants typed a statement of consent - but getting participants to set aside time to read and 'sign' these forms was difficult. Data from non-guild players is incorporated anonymously as overheard rather than solicited information. The dissolution of my guild(s) over time made sharing the results of this research with participants more difficult (guild forums and websites were no longer available by October), but where possible I have emailed summaries of my findings to participants. A summary was also posted in the TERA official forums, to make it available to the wider player community. En Masse Entertainment were notified of and consented to the research beforehand, and a copy of this work was shared with them.

Throughout this thesis I represent myself/my character in chat logs as 'Elizabeth'. This was not the name of my character, the high elf priest, and thus not the name by which TERA players tended to know me. I use it in place of my character name because that name does not pass what I call 'the Google test'. Entering my character 
name into a Google search at the time of writing produced records of the guilds I was involved with, thus breaching the confidentiality of the research (cf. Boellstorff 2008:83, Suler 2000). This simple test was also a useful way to check that quotes could not be used to identify informants, except where they were taken from publicly accessible forums (which I deem acceptable). Character names have been replaced with pseudonyms throughout, as have server and guild names. Character pseudonyms are taken from the story and world of TERA itself, 3 in order to avoid replacing the diverse names players were known by with clumsy synonyms which could be too easily linked to the originals. The server I played on, Sienna Canyon, is named after a zone in the game, as the real TERA servers were.

In general, the manner in which I conducted this research was in line with the principles laid out by the American Anthropological Association (AAA 2012). Although the online site required some further interpretation, ethnographic methods have always been flexible by necessity and TERA was not such an exotic site that it required major changes to these methods. Treating the site with the same ethical concern I would give to face-to-face research was a basic (and to my mind utterly vital) acknowledgement of the fact that, mediated or not, I was dealing with real people through the medium of the game.

\footnotetext{
${ }^{3}$ The two most prominent, 'Elleon' and 'Jelena', are the names of heroic characters that players fight alongside in the game. Most others belong to minor characters that could be interacted with for specific quests or services.
} 


\section{Interlude: Sinestral Manor}

$25^{\text {th }}$ May 2012

"Any healers please for SM? One more then ready to go!"

The plea comes across the 'looking for group' chat channel, which can be heard by the whole server. I notice it because she asks for a healer specifically, which is the role I fill, and because mention of 'SM" (Sinestral Manor, an instanced dungeon) causes a pang of guilt. My character is level 38, and I should have completed SM long ago, but I have been avoiding it because instances require a group and a substantial investment of time. There is also a down side to playing a healer which I had not considered playing alone, I tend to focus on attack skills (so that I can actually kill things), but in groups a healer must focus on just healing the others, something that I haven't actually had much practise with. My guildmates are working on their own characters, at much higher levels than me, and I don't want to bother them.

Freya's message prompts me to get over my nervousness about others depending on my inexpert healing skills. "I'm a level 38 priest, but I never did run that instance, haha," I whisper her (sending a message that only she will see). "I could be there in a bit?"

"Ok please come!" she replies. "Tell me when you're here inside 'cause there's PKs." 'PK' stands for 'player killer', one who kills other players, always a risk on PvP servers. It takes me a while to travel to the Manor's location from where I am, but I assure Freya I'm on my way as fast as possible. She has a group assembled and seems impatient to start. "How long 'til you get here?" she whispers me.

"Have to fly but I'm about the grab a pegasus," I reply - the winged horses are the main way to travel between major cities, although they take a while. "If you're impatient go ahead..." I'm a little annoyed at her hurrying, given that I thought I was doing her a favour by coming.

When I make it to Sinestral Manor, I find Freya waiting outside. Before we can say anything further someone from her group gets into a fight with a monster that was 
roaming nearby, and we all leap into the battle instead. I'm glad of the chance to try out my healing skills and practise targeting people in the confusion, skills I'll need in the future. When we have dispatched the monster, Freya turns me to and says "thank god, I really thought you were coming here to PK me Iol."

It hadn't even occurred to me how suspicious it must seem that a higher level character would come back to a lower level area like this, where others preyed on the weak. I laugh it off, but file the lesson away for future reference. Freya has assembled a full team of five people, including another priest who turns up. "Two healers? We'll never die," Freya declares. One of our companions, Kimuk, retorts "it will just take longer to kill."

Together we enter the portal into the instance, a copy of Sinestral Manor inhabited only by the five of us - and any number of hostile creatures to kill, of course. We start carving our way through them as Freya, who has obviously done this before, gives advice about where to go and what to do. Soon we approach the first major 'boss' enemies in the Manor, a vampire Countess and her Count (who is in the next room). Carefully, we eliminate the other enemies around the Countess first so that we can fight her alone.

The battle is a close thing - two players are killed, although we are able to revive them. Freya and I push the attack at the last minute, knowing that either we take her down, or we're all doomed. Thankfully, we finish her off. My frantic healing attempts were not able to keep up, but there are no hard feelings, as everyone is more aware of their own mistakes. Apologies are made all around.

As we head into the next room where the Count awaits, the image of Freya on the side of the screen suddenly blacks out, and she disappears; she has become disconnected from the game. Someone in our group moves in on the Count without realising this, forcing us to start the fight without her, but luckily I notice her reconnect partway through and she quickly joins the fray. After that, this battle goes more smoothly than the last one, and when it's done those who know the way lead on to the next location. 
There are a series of tasks and fights which we move through without major incident, progressing toward the final boss. When we get there Kimuk triggers the fight, and the game cuts away from us for a short video scene showing the monster, called Malgarios, making a dramatic entrance. When our normal perspective is returned, however, Freya and I find ourselves at one end of the hall while Kimuk is running away towards the other end. The boss is bearing down on us and we can't fight it on our own; there is some confusion, and panicked typing as we try to ask questions while also keeping ourselves alive!

"Run here," Kimuk says, brusque to cut down on typing. He is at the opposite end of the hall. The rest of us eventually re-orient ourselves and disentangle from the boss's spidery legs, fleeing towards him. Kimuk leads us into a smaller room, back in the direction we had come from.

"Where are you going?" Freya asks.

"In here," Kimuk replies unhelpfully.

"Why? Can he not fit?"

"He summons shit," Kimuk explains. "They can't get in here."

Soon enough the beast catches up to us. Fighting Malgarios requires all my concentration, and as healer most of what I do involves watching the others' health displays, topping them up when they fall low, and purifying them when they turn from healthy ally blue to the purple which signifies effects like poison. Half the challenge is targeting the people who need help as we all circle and dodge around the boss's attacks. Where normal combat is methodical and sometimes dull, this climactic fight raises adrenalin - more so with the knowledge that other players are depending on me to do my part.

At one point Malgarios scuttles out of our little room and back into the main hall. "Hey you!" Freya calls after it.

"Don't chase," Kimuk cautions, "he will come back." So we wait, and a notification flashes onto the screen: 'Malgarios's shriek summons its dark offspring.' We see no offspring, though - this must be what Kimuk was talking about earlier, they don't follow 
back this way. Not having to fight a horde of smaller monsters simultaneously certainly will make this easier! In our brief respite we chat a little. "I'm really trying to dodge," Freya says, referring to the most important and most challenging aspect of her class, the warrior. "It's good," I say, "healing for you isn't too hard." If she were bad at dodging, I would need to heal her constantly, but I have found it quite manageable so far.

Malgarios comes back after us and the battle is joined once more. We've more or less got our techniques down now, it's just a matter of wearing away its health, which is shown as a percentage as well as the usual red-filled bar. As this drops to $3 \%$ remaining I start to get excited to see our final victory. At $0.7 \%$ health remaining, though, disaster strikes! I experience extreme lag, none of my skills activate as they should, and everything stops moving. The signs are familiar, so although I groan I'm not surprised when I am disconnected from the game, just as Freya was earlier. Hurriedly I reconnect as quickly as possible, and succeed in getting back to the manor, although the room is now empty.

"Frickin' internet cut out!!! Grr sorry," I type to my group. I'm not usually one for multiple exclamation marks, but its seems an appropriate way to express the frustration of the moment.

"Did you get everything?" Kimuk asks.

I check. I didn't get a chance at any good items that Malgarios might have dropped, but the quest that I needed to complete has registered as completed. I thank my companions and use a teleporter to get back to the main, shared game world where we started. As the others start to leave the group one by one (with polite thanks) I do the same, dissolving the small, temporary team we formed for this one place - but it was fun. 


\section{The Life Cycle of a Game}

As a new game entering a market increasingly saturated with quite similar MMORPGs, TERA was competing for the attention of players who were happy to sample a large number of games, but tended to only intensively play (and pay for) one at a time. As Debeauvais et al. (2011:180) state, for online games to be profitable the goal is not simply to sell many copies, but to keep players engaged and playing as long as possible. This chapter provides a historical context of TERA for the four month period in which I conducted field research. Although in 'real world' terms this is a short period for a historical account, TERA went through many major changes in this time. Online games are always works in progress, constantly updated, and each participant experiences a game at a particular point in its development. Players themselves used the language of life: a game open to players was "live", while a server could be said to be "dying" (or a game "dead") if it has few players. In recounting the rapid rise and decline of TERA as a 'life cycle', both of the software and of the associated community, I wish to highlight two things: the amount of change and updating the game underwent in its early months, and the attitudes of players who had seen, played and abandoned many similar games before. Both elements are important in framing player actions and agency.

\section{Temporality, History and Migration Online}

"WoW was an incredible experience when I first played it...Now I can't get myself to play it if I try."

(Area chat)

It is extremely important to bear the passage of time in mind when talking about online games, because they are subject to frequent (sometimes dramatic) updating and changes. Although all cultures are subject to change over time, from outside or from within the group, the process is rapid in online game worlds and most often due to very deliberate decisions. Nardi states in the prologue to her book that "WoW [World of Warcraft] players will recognise that references to the game belong to a moment in time" (2010:6), and in fact WoW has already received two major content expansions since the book was published. Games can be not only updated and 
changed but shut down entirely, becoming inaccessible if companies decide not to support servers any longer. Bainbridge (2010:4) notes "there exists no library of outof-print worlds, nor academic publishing houses that reissue old games. Yet already, some of the very most interesting examples have ceased to exist.” With more and more new MMORPGs being released - and vying for the attention of the same players - the metaphor of a 'life cycle' becomes apt. New games have periods of vulnerability as they grow and compete, trying to be successful enough to survive in the long term.

Others have noted the relevance of history and temporality in studying an online society. Boellstorff's seven year investigation of Second Life saw the game grow from five thousand to over ten million registered users (2008:83), which changed the dynamics of the world significantly and introduced new issues. When Pearce (2009) studied the 'refugee' communities of Uru players who had migrated together to other virtual worlds, her position was partly defined by the fact that she had joined them after Uru was shut down and had never experienced the group's original world. Uru was known to her through imitations, references and informant accounts, but she had not been present for that stage of the group's history. Still others have witnessed the life and 'death' cycle of games they have studied (e.g. Papargyris and Poulymenakou 2009, Zabban 2011) and analysed players' (and developers') reactions when a world was shut down. Jakobsson (2006) recounts the lifetime of a game character in EverQuest, highlighting how a player's experience of the game changes with their advancement, with 'endgame' play being dramatically different to the introductory levels in terms of social dynamics as well as game tasks.

As a case study, TERA is interesting not because it proved to be one of the biggest, most popular, or most innovative games, but because it went through a life cycle typical of any number of similar games, including its contemporaries. It had a period of anticipation and a rush of enthusiastic play at launch, followed by a shaky transition to 'endgame' and, eventually, a gradual loss of interest on the part of many of the earliest players. TERA players had experienced the same cycle elsewhere and often (with similar durations4), and commented on it even as they lived and enacted

\footnotetext{
${ }^{4}$ Online game blogger 'Ravious' describes MMORPGs as having "baby fat" at launch which drops away after initial interest ('Ravious' 2012).
} 
it. A very large proportion of casual public chat in-game centred around the comparison and criticism of competing MMORPGs or games which contained similar elements, such as the single player Skyrim (Bethesda 2012). Players were well-informed about upcoming games and had strong opinions about others that they had played, as just a few quotes will illustrate:

"I'm sorry but SWTOR's5 levelling was the best ever...it just didn't have anything going for it after that :(" "agree...SWTOR levelling was excellent, the end game sucked"

"There's a game called Secret World coming out soon, seems promising, lots of pvp apparently...I don't know much about its mechanics though"

"Secret World looks like a flop in making. Not trying to troll it or anything, just doesn't look like it really provides anything outside of whats already here in the mmo market"

"Imo ['in my opinion'] they released D3 just like they released SC2. A half finished game"6

Most of my guildmates played other multi-player games alongside TERA even if they only maintained one paid subscription, and every TERA player I spoke to had played other MMORPGs in the past. In fact, the majority had played many of them, perhaps reflecting TERA's position as a less-publicised niche game which only existing fans of the genre were likely to hear about.7 Whatever the reasons, it was clear that TERA did not exist in a vacuum. A number of my server's largest guilds were trans-game entities, groups with their own independent websites and local chapters in a number of different games. Players moved to TERA as the latest in a long line of game worlds where they had joined, experimented, and in most cases ultimately grown bored. As a new game which claimed to be innovative, there was hope that TERA might prove to have more lasting appeal.

\footnotetext{
${ }^{5}$ Star Wars: The Old Republic.

${ }^{6}$ The games referred to here are Diablo 3 and StarCraft 2, both by developer Blizzard of World of Warcraft fame.

${ }^{7}$ There is also a selection bias, in that I spoke to players on a PvP server, who generally considered themselves more 'elite', skilled and experienced than other players and thus were more likely to have played other games.
} 
It would be misleading to suggest that TERA players had no attachment to the game. Many were quick to defend it against complaints, reiterate what it did well, and phrase their own criticism supportively, in forms such as "TERA would be a great game if they would just..." Nonetheless, the threat of players jumping ship at the next major game release was ever-present, and highlighted by public discussion of the competition. I will return to the significance of this in chapter six.

Celia Pearce has coined the term 'ludisphere' for "clusters of intersecting and overlapping magic circles within the larger constellation of networked play spaces...which exists in the larger frame of "real life"” (Pearce 2009:178). TERA can be fruitfully considered as part of an online ludisphere, in a 'cluster' of massively multi-player, competitive/co-operative, combat-based games in fantasy settings which share certain design conventions. Large, well-established games like World of Warcraft (Blizzard 2004) or Lineage (NCsoft 1998) - which have for the most part been the focus of existing research - can be studied as relatively self-contained systems, but they are connected to the MMORPG ludisphere by the movements of players. Others, especially new games like TERA which must attract and retain a player base, are much more at the mercy of what scholars and gamers alike have called 'churn'. This constant turnover of people joining and leaving is an effect of player agency operating where players enjoy freedom of movement and information across this ludisphere, and could change their market choices regularly. With that in mind, I offer an overview of the major phases of a 'life cycle' that TERA and its player population shifted through during the game's first four months.

\section{The Road to Release}

"And ding, lvl 20...time for a late night taco bell run and then back to the grind :)" (Guild chat)

The first version of TERA was launched in Korea in February 2011 by new developers Bluehole Studio, with a subsidiary company called En Masse Entertainment responsible for 'localising' the game for North America (Business Wire 2010). As far as North American users were concerned, En Masse ran the game they played. The localisation process included not only translation but also various adaptations of 
content and gameplay for a new market. It was part of the plan for TERA from the beginning (with the game announced as in development for a Western audience even before the Korean version was available to players) rather than a later expansion for a successful product. As an En Masse representative told a player, who posted the response in the forums:

"KTERA is actually a different product than the version released in North America. The North American version has gone through a full "Westernization", which includes a lot more than just translating text. In fact, all of the lore in the North American version was written by writers here at En Masse, so that it caters to a North American audience. There have also been changes made to the game-play mechanics, because gamers in North America have different expectations for an MMO than gamers in Korea."8

Many MMORPG players followed the industry closely and tracked games that were available or in development. In TERA this was reflected in the preoccupation (demonstrated above) with discussion and comparison of other games in public chat channels. This tendency led to TERA being known and anticipated by North American players long before its actual release there. Players tend to only maintain one monthly game subscription at a time (Pearce 2009:267), transferring the expense to another game if they become bored. TERA's claims of "true action combat” and its striking artwork helped to set it apart from the many other MMORPGs seen as, in gamer terms, 'WoW clones'. When TERA's North American release drew closer, En Masse Entertainment offered special benefits (including early access) to people who pre-ordered the game, and many interested players ordered months before an official release date was even announced.

Between February 10 th and April 8th 2012 (before I joined the game community), a series of five 'closed' beta tests were held to which pre-ordering customers had access. Each test lasted for a weekend, after which the servers were closed and the game was once again made inaccessible to players while En Masse made any changes they deemed necessary. Although ostensibly 'closed' and thus exclusive to a certain number of players, anyone who paid for a pre-order could participate. Additionally,

\footnotetext{
${ }^{8} \mathrm{http}$ //tera-forums.enmasse.com/forums/general-discussion/topics/Archers-and-Westernization (accessed 14/01/13).
} 
players were not bound by the non-disclosure agreements typical of closed testing for an unreleased game, meaning they could talk and post freely about their impressions. The test weekends allowed players to download a version of the game and try it out, experiencing first-hand what had been advertised and increasing fans' eagerness for the full launch. At the same time En Masse Entertainment could test both the game and the underlying technology with a larger pool of players, who asked for no pay and happily shared their opinions. Giving players the freedom to discuss their testing publically made the closed beta tests as much about publicity as about polishing the game.

Despite the temporary, provisional nature of everything in the virtual world during this testing, a game community did begin to develop. Guilds were formed, and recruited among fellow beta-testers both in the game and through the official TERA forums, which included a sub-forum dedicated to guild advertising. This was filled with posts from this period including descriptions such as "we want to be one of the most feared AND respected PVP guilds on the server", or at the other end of the spectrum, "an adult, laid back, community oriented environment free of drama and real life nonsense". All closed beta players had pre-ordered TERA and expected to play it when it was released, and so this was a time for players to master the gameplay, put down roots in the community, and establish their guild reputations. The TERA players that I met were highly competent, knowledgeable and experienced, and had a good idea of what to look for in a guild. 'Bellicus', the guild I joined, were already quite established when I found them at the end of closed beta testing despite not actually having characters or a game to play yet.

Over the weekend of April 19 th, TERA shifted to a brief period of 'open' beta, during which anyone could download the game client and play for free. Open beta served to promote the game to potential players who were not yet committed to purchasing, as participating required no long-term commitment. For players who had pre-ordered and participated in the closed beta tests, the open beta period was significant because it was the first time the characters and progress they made would persist to the official release date. Guilds created in-game by pre-purchasers at this stage were formally saved, making this 'the real thing'. Although the maximum level for characters was to be 60, En Masse Entertainment limited pre-ordering players to 
level 38 during open beta. Experienced players treated this maximum as a target and rushed to reach level 38 before the end of this period, some playing intensively to clear content they had already completed four or five times over during the closed beta tests. Members of my guild wanted to get as much done in this time as possible, often prioritising game progress over offline commitments such as work:

Rendalf: so tempting to take tonight and tomorrow night off [work] so i can have a 5 day off to play lol

Orlaith: i already did, lol

Deigan: i beat you to it already

Deigan: i already called tomorrow and tuesday off

Rendalf: wow nice guess i should fall in line and join ya

Rendalf: i need to catch up is the reason i want to take a couple off

To play on equal footing with other guild members and their opponents, a player such as Rendalf had to keep up with their pace, to 'catch up' to friends who were already at higher levels. As a consequence of these opportunities for early access, a large proportion of the dedicated fans who pre-ordered TERA entered the game's official release stage already almost two thirds of the way to the maximum possible character level, and thus the 'endgame' which was intended to occupy them in the long run. 9

\section{Game and Endgame}

"You guys know as well as I do that every game is like two different games, levelling and endgame."

(Guild voice chat)

I pre-ordered TERA after the closed beta tests but in time to participate in the open beta. Most of the members of my guild had played through the early stages of the game so many times that they could easily complete them within two or three days and progress into previously unseen areas. Even playing alone, game content was

\footnotetext{
${ }^{9}$ This is potentially misleading as gaining levels became a more difficult and extended process at the later stages of the game; progressing from level 10 to level 20 could be done orders of magnitude more quickly than progressing from level 50 to level 60, and at 60 the 'endgame' was intended to continue indefinitely without more levelling. Nonetheless level 38 was a significant way through the more structured content.
} 
quite easy to complete and gaining levels for the most part only required the investment of time. There was a rough progression through which players were guided by story quests, which told the game's central story through a series of linked objectives - as small as speaking with a certain character, or as big as overcoming a powerful enemy. Fighting and defeating enemies (usually cartoonish or monstrous) of appropriate strength earned players experience points which contributed to reaching higher levels. Guild members might move through levels with a group or play at their own pace, using the guild as a resource when capable allies were required or simply for socialising while playing 'alone'.

'Levelling up' in TERA could be a tedious activity. As a priest, I could reliably keep myself alive in most cases (except against other players!) but dealt relatively small amounts of damage with attacks, meaning it took a long time to work through enemies. Questing - which took up by far the majority of my play time - mostly consisted of receiving orders to kill a prescribed number of a prescribed kind of foe. I would find the area where these foes milled about nearby, and pick them off one by one until the quest was fulfilled. The process had to be repeated many times over to gain a level, and most solo fights were not particularly challenging. From level 1 to around level 50 in TERA, little changed. As players reached level 6o, however, there was a shift from the treadmill-like progression through zones and quests to a less linear and less directed form of gameplay. Instances, which required a tactically balanced and well-co-ordinated group, became the main form of occupation for players.

Instances were repeatable, designed for five person groups, and separated off from the shared game world through the process called 'sharding' (Bainbridge 2010). As Bainbridge explains, "the term instance implies there are two or more versions of the same section of one world running simultaneously" (2010:26); what each group of players experiences is one 'instance' of the area. Unlike in the open world, a group in an instance would not encounter other players, as any other group wanting to attempt the instance would have their own version created for them. This allowed large numbers of players to attempt the challenges within - and earn the rewards for completing them - without groups being in direct competition with one another. Instances, or colloquially 'dungeons', consisted of enemies tuned to require five 
people, and a series of unique 'boss' enemies with the potential to drop valuable items when defeated. My guildmates tended to repeatedly play through the instance most suited to their current level, seeking the best equipment available in one place before moving on to the next.

I played on a PvP ('player versus player') server, where guilds could declare war on other guilds and individual players could declare themselves 'outlaws' and attack other players while roaming the game world. Because of this, reaching level 60 was a particularly important goal for players on my server. A fight between two characters of different levels would almost always end in favour of the higher level character, and so falling behind made one vulnerable. Guilds also had an interest in encouraging their members to strive for the maximum level, as characters killed in a guild war cost their guild points. To be competitive in PvP, players had to keep up with each other. This meant that the more determined guilds could set the pace and force others, like my guild, to put in more effort or fall behind. One's character's level reflected how much one had played the game, but in this early period in particular a high level indicated a dedicated and efficient player, likely experienced and skilled. ${ }^{10}$ With many characters already at level 38 by TERA's official release date and many players under pressure to reach the maximum so that they could participate capably in $\mathrm{PvP}$, it was only two weeks before prominent members of my guild began to "hit $60 "$.

As well as the fear of being left behind or becoming a liability in PvP, there was a sense among TERA players on my server that the 'real game', the more challenging fights and more worthwhile rewards, only became available after a character reached level 6o. Amidst the frustrations of uneven fights between warring guilds I was told "soon enough everyone will be 6o. That's when we'll see who the real top guilds are." The instances targeted at level 60 characters were significantly more challenging. Much of the levelling process leading up to these could be considered as a form of training, especially given TERA's atypical combat system, which required more

\footnotetext{
${ }^{10}$ It is worth noting that my own character was notoriously behind the average level in my guild. Although research activities occupied some of my play time, I freely admit that my slow levelling progress had more to do with the fact that I was not as enthusiastically dedicated to the game itself, nor was I as skilled with its systems or the meta-game of efficient levelling as my guildmates were. They were accepting of this (settling for teasing me) and I was not the only member who progressed more slowly and casually; Bellicus were an easy-going group. In more 'hardcore' guilds, my slow progress might have been grounds for expulsion.
} 
attention and skill than other MMORPGs (or so players frequently claimed). This attitude reflects a widespread focus on 'endgame'. This term was oft-used and illdefined, but it referred to game-play options available to a character once they had reached the maximum level. Once play was no longer in the pursuit of the next levelup, something else was needed to keep players interested enough to continue their subscriptions. Online games could not concede a real end point; they relied upon players developing a continuing relationship with the game (and thus continuing to pay for it) to be profitable (Debeauvais 2011:180).

Members of my guild made clear that they were aware of this very early on. Discussing the game before its release, a guildmate commented that "recent games don't seem to understand how quickly people will level, or be prepared for them to get to max so quickly" - an insight which proved to be prophetic. Insufficient endgame was a common criticism that TERA players made of other MMORPGs, notably Star Wars: The Old Republic (BioWare 2011) because it was a recent release when TERA launched. Despite the frequency with which this accusation was levelled at games, what constituted good endgame was far from agreed upon. Player-driven activities and conflicts were popular because they could be more dynamic and involving than anything programmed, but they were difficult for developers to reliably design. Nonetheless, developers were the ones held responsible if players got bored. They were the service providers, and their customers paid to be entertained, not to make their own entertainment. Even as players asserted their agency in directing the development of the game, they identified as consumer agents and expected their fees to pay for others to make the changes they wanted to see. I will return to this aspect of the developer-player relationship in chapter six.

\section{Dungeon Imbalance}

"Yeah no one is playing this game anymore."

"It's not because people don't play, it's because people don't play tanks." (Area chat)

TERA provided an 'instance matching' tool (sometimes called a 'dungeon finder' from World of Warcraft parlance) as part of the game which allowed players to list 
themselves as available for one or more instance. The game then automatically brought applicants together into groups with a diversity of character classes to fill necessary roles in combat: healer, 'tank' (group defender), and damage-dealer (called 'DPS', from 'damage per second'). The automatic instance matcher formed temporary, largely impersonal groups of players who viewed each other instrumentally, as means to an end. Despite the fact that such groups were widely considered to be less effective as well as less fun than guild groups, use of the instance matcher was extremely prevalent. It offered a number of incentives, such as teleporting characters to the right location rather than requiring them to travel there. My guildmates occasionally formed partial parties and applied to the instance matcher for additional members, but just as often they applied individually, apparently valuing the convenience of letting the tool organise for them.

As a large proportion of the server's players began to reach the upper levels of the game, certain inequalities began to emerge among the game's combat classes. Although all classes had their advocates and detractors throughout my research, in this period players discovered that their initial class choices could have unforeseen consequences for their participation in the game. The first and most important development was that lancers became absolutely central to instance grouping. One of eight possible character classes, lancers were excellent defensive characters and filled the role of 'tank' in a group, placing themselves in harm's way to free up less hardy characters. In theory another class, the warrior, could work as a 'secondary tank' (according to the blurb at character creation). In practice, however, most players felt that warriors were only effective tanks in the hands of very skilled players, and they were often not trusted with the role.

Not only did players prefer a lancer to a warrior when putting together a group, but the automated instance matcher did not actually classify warriors as tanks. This resulted in a serious imbalance in the pool of potential group members, as the eight possible classes were separated into two healers, five damage-dealers, and only one tank class. When putting together a group the instance matcher always sought at least one character for each role, and so it came to pass that no group could form through this tool without a lancer. As most players were using the instance matcher to find groups, the need for a lancer created a bottleneck that stalled group formation 
and extended the time that players spent in 'queues' waiting for an opening. Although it was possible to continue playing while queued through the instance matcher (one of its perks), there was no meaningful reward for killing average monsters after a character reached the maximum level. Because it was easier and safer, players at level 60 tended to just wait idly in towns until their "queue popped" and they could join an instance.

Damage-dealing classes, who were the most common and thus in the least demand, had the most trouble with finding groups. Because lancers, by contrast, could instantly find a group to work with through the instance matcher, the tool suited them, forcing others in turn to become more dependent on it. As a guildmate explained to a newer member, "I know the queues suck as dps but you wont be able to find a tank using chat cause tanks just DF [Dungeon Finder] insta-queue." Lancer players gained power over others because everyone needed them, upsetting an idealised balance in which classes were different but equal, issues which will be expanded upon chapter five.

The difficulty of finding a lancer to fill the tank role so that a group could progress added a lot of dead time to an already somewhat sparse endgame. Level 60 players had a number of instances they could run, but spent hours doing very little as they waited for a group to form. PvP opportunities, a major part of this game's appeal for many of my guildmates, soon proved difficult to find. Towns were safe zones where no player could kill another, but queuing characters had little reason to leave that safety, so there were fewer potential targets in the open world. A more organised form of group-based $\mathrm{PvP}$ was much desired by players, but such a feature was absent from the game at its release, scheduled to be added in the form of 'battlegrounds' sometime in August. For some this was a sign of better things to come, but for many August (three months after launch) was too late for a feature common in other games. Alternative forms of progress were available to level 60 characters who wanted to gain an edge by improving their equipment, but such activities did not provide an effective alternative to continuing to slay the same virtual monsters - they just provided reasons to continue doing so without levelling up. 


\section{Patches and New Features}

"So what does everyone do at endgame in this game? Because I'm kind of afraid to hit 6o."

(Area chat)

While these options proved unsatisfying for many, alliances and rivalries between players had the potential to keep people engaged and involved (cf. Jakobsson and Taylor 2003). TERA's political system was one of the more unusual features advertised before the game's launch; it allowed guild leaders to run a province in the game world if they were elected to the position of 'vanarch'. However, running for vanarch proved to require a lot of work from players. Not only did vanarch hopefuls run publicity campaigns across forums and in the game world before the monthly elections, but guilds with a leader in office had to spend much of their time completing tasks and gathering resources in order to maintain their province. The system offered very little in the way of in-game rewards for this effort, with its main benefit being fame and prestige for a guild. These was only valuable if players considered them so, and once some groups on a server began to lose interest in the vanarchy, winning rapidly became less of an accomplishment. As one guild leader put it:

"It's a lot for vanarch...the only thing you get out of it is money, is gold...and you get a little faster horsey...But the amount of work involved, and the amount of bullshit you have to go through, and all that stuff, I don't think it's worth it."

If major guilds opted out, the atmosphere of rivalry that might provide a social incentive to win could not be maintained. On Sienna Canyon, candidacy dropped from almost every notable guild leader in the first election (some portion of over a thousand guilds created on the server), to only fourteen candidates standing for fifteen provinces by the fourth election. By this point, when anyone who could afford the cost of applying to run could be guaranteed a province because of lack of interest, vanarch positions had lost their prestige value. 
Although vanarch elections inspired a number of player-run events, including both $\mathrm{PvP}$ combat tournaments and non-combat events such as hide and seek in the game world, the outcome of the elections relied more on voting alliances between guilds than on campaigning to the public. Provinces were separated into three continents with each player getting a vote for each, so large guilds could trade votes, electing their own leader in one continent and their allies in the others. Not everyone was pleased with this take on democracy; one guild briefly advertised their campaign in public chat channels by suggesting "Vote Soldier's Honour so we can win against the guilds that have more votes despite never advertising because voting alliances suck." Guild warfare and vanarch competition became devalued, and players lost interest in these optional aspects of the game. This was both a cause and an effect of the weakening of guilds like Bellicus, as described in chapter four, as the state of the game and individual group dynamics fed back into each other.

As time went on and players reached the obvious goal - the highest level - they started to look around more critically for something to keep them in TERA. This stage of TERA's life cycle marked a widespread shift from 'levelling' to 'endgame' among players. TERA's particular difficulties may stem from the fact that the problems with endgame were encountered less than a month after release, before much adjustment could be made. Players participated in a ludisphere that constantly offered new games, and were accustomed to easily moving between game worlds. They moved through the levelling phase of TERA swiftly and reached a point of evaluation: is it worth staying here, to the exclusion of other games? The game continued to evolve and develop, often in response to player criticism - but this took time, whereas many players made their judgement at an early point and then stopped playing the game. These players never experienced later stages of TERA's 'life cycle' and likely moved on to a new MMORPG, or back to an older one.

Subscription fees meant that it cost players to retain their access to the world, and those who felt that they were no longer getting their money's worth cancelled their subscriptions. Some did so after only one month, having had their fill within the free month's subscription included with a game purchase; such players had an incentive to leave then rather than pay for another month in which they might not have anything to do. For many others, leaving the game was less a conscious decision and 
more a gradual disengagement. People logged on less often because they were less motivated to play or improve their characters. This led to less active public chat and less people around the game world. The world quickly began to feel noticeably emptier, and where once an area would have multiple channels ${ }^{11}$ to prevent overcrowding, now one might only see one other player in an hour even with a single channel. This fed back into a cycle which drove people away because of the lack of people, although many who still wanted to play took the step of shifting to a busier server rather than leaving the game altogether.

En Masse Entertainment recognised that finding groups through the instance matcher had become a problem, one only exacerbated by falling player numbers. In response to their own observations and to player feedback (in the form of quite bold criticism, to be discussed in chapter six) they worked to make changes to the game. The warrior class was acknowledged to be insufficient in the 'tank' role, despite being advertised as suited to it. Working in parallel with efforts in the Korean incarnation of TERA to address similar problems, they began to allow warriors the option of listing themselves as tanks when joining an instance matching queue. ${ }^{12}$ Warrior had consistently been one of the most popular classes throughout closed and open beta testing, so adding warrior characters to the pool of tanks would greatly increase the odds of finding one for a group. Unfortunately, instance matching is only one element of a complex game, and making changes to warriors that helped in one area could unbalance gameplay in another - for example, many objected that allowing warriors to effectively attack and defend would make them too powerful as individuals in PvP where other individuals had only one specialty. En Masse's solutions had to be not only mechanically sound, but also perceived as fair to all players (see chapter five).

While working on finding a solution to the problems with instance matching and warrior skills, En Masse gradually introduced new content to the game. 'Nexus events' were open to high-level characters and happened in hared zones rather than

\footnotetext{
11 'Channels' were copies of an area in the game world within a single server, which players could freely switch between. They spread players out to give everyone space to play in a busy zone. Switching channels could be used as an alternative to waiting for a desired enemy to 'respawn' (be generated again after being killed) - or in combat with other players, to avoid PvP or escape retribution after a player kill.

${ }^{12}$ Players discuss warriors as tanks in the Korean version and the implications for North American updates here: http://tera-forums.enmasse.com/forums/general-discussion/topics/Warriors-in-the-dungeon-finder (accessed 14/01/13).
} 
instances - allowing more people to participate together, but causing problems as the servers strained to keep information flowing between so many computers. On August 21 ${ }^{\text {st }}$ the much-anticipated 'Queen of Argons' update added new instances and the much-awaited 'battlegrounds', a form of player vs. player arena. 'Queen of Argons' (named for the most powerful new enemy added to the game) addressed many of the complaints that had been raised, but by the time it was available, many players had already left TERA. En Masse needed to entice people with inactive accounts to return, as well as satisfy those who had stuck with their game despite its issues.

Population remained an issue. On my return visits during August, the game world still felt empty. Sienna Canyon server was accepted to be 'dying', although many players still held out there rather than shifting their characters to busier servers. Server merges were looking increasingly likely. This tactic - when a company condensed players' characters into a smaller number of servers to increase the population of each world - was common in MMORPGs, but was interpreted by players as a sign that a game was failing. Early merges suggested an initial burst of interest in a game followed by a mass abandonment by bored players, which did not bode well for a game's longevity. TERA players had been able to shift servers for free if they chose to, but on September $3^{\text {rd }}$ (just at the end of my research period) TERA announced a dramatic merge, reducing eleven servers to only three - one PvP, one $\mathrm{PvE}$, and one role-playing server - in order to re-invigorate the game by bringing players together. ${ }^{13}$

\section{Conclusion}

This account of a period in TERA's history, and of the ludisphere that it was part of, highlights trends in the practices of both players and developers which will be explored anthropologically in the following chapters. Although it is important to bear in mind that TERA continued to be updated and adapted after the period of my research, these first months of its 'life cycle' encompassed a startling amount of change and 'churn' as players moved into and away from the game. Players were highly mobile within the ludisphere of MMORPG gaming, and they recognised the

\footnotetext{
${ }^{13}$ Fall Producer Letter, http://tera.enmasse.com/news/posts/tera-pd-letter-server-merges (accessed 05/09/12).
} 
life cycle of the game from experience even as they enacted it, but their identification with a consumer ethos led them to deflect all responsibility for the state of the game back onto En Masse Entertainment. Numerous other games offered similar entertainment benefits, and many players had little patience for gradual adjustments that would allow the game (a product crafted by people who, after all, needed time to work) to become more like what they desired. As only one game among many possibilities, TERA struggled to hold the attention of people who were accustomed to games boring them eventually. The case of 'Bellicus' and its leaders in the following chapter will show that even players who invested significant time, resources and emotion into TERA were prone to leaving the game once they had expended its immediate entertainment value. 


\section{Interlude: Guild Conversation}

(From field notes, June $15^{\text {th }}$ 2013)

Elizabeth: Hello :) Just checking in (I will have to go again for lunch soon)

Popomin: lunch.....?

Elizabeth: Haha I live far away. New Zealand.

Popomin: oh

Popomin: Iol

Elizabeth: It's the middle of Friday here :P

Popomin: oh

Popomin: wow

Elizabeth: Iol

Kiriya: HES FROM THE FUTURE!!!

Popomin: 15 hour difference

Kiriya: what will i do tomorrow?

Kiriya: TELL MEEEEE

Jelena: SHE

Jelena: Elizabeth is a she!

Elizabeth: She, and yes we live in the future but are sworn to secrecy lest we break spacetime

Jelena: like jelena

Kiriya: SHEEEEEE is still from the future

Popomin: haha 


\section{The Life-Cycle of a Guild}

Guilds in TERA, as in most MMORPGs, were the main form of persistent group identity among players. To make friends, find people to regularly play with, or attempt challenges which required a well-co-ordinated team, players joined a guild. They were formal institutions in the game, with tools to help leaders manage them, and a character's guild name always on display. Although the guild I joined displayed a lot of enthusiasm and ambition, it was also at the centre of numerous conflicts and interpersonal dramas. By the end of my four months playing TERA it had been formally disbanded. In this chapter I will recount the history of this guild, called Bellicus, and through it the everyday relations and turbulence that happened within the broader context outlined in the previous chapter. While the state of the game was largely out of their hands, players could set the rules for their own guilds and make choices about what groups they associated with. Relationships in TERA were often entered into whole-heartedly but abandoned when no longer desired - paralleling players' approaches to games themselves. In a setting where all relationships were voluntary ones, players chose who to play and associate with through a market mentality. This emphasis on free choice - to enter or to leave a relationship without obligation - is a reflection of the consumer agency that TERA players embraced in other aspects of their play.

\section{Formation and Leadership}

"You know with a new MMO, the whole thing is you need to have a strong start, just to keep the momentum going, to be successful because you know people leave, people quit...you need the numbers, you need the thing going." (Elleon, interview)

'Bellicus' was founded long before the game's official North American release. Its two founders had met and become friends in another MMORPG, and told me they had known each other through that game for about a year. They had eventually become bored with what their current game had to offer and began looking for a new one. Having followed the news of TERA's development and localisation for North America, they decided that it looked promising and began preparing to start a new 
guild from scratch for TERA's release. After strong participation in beta tests and advertising on the forums, the guild entered TERA's official release with around 80 members and boasted 160 at its largest, making it one of the larger guilds on our server. Having infrastructure for the guild (such as an external website, forums and an online application form) organised early showed that the leaders were serious and dedicated; as one early recruit commented, "It's great to join a group that already has the polish on and is ready to go day one!" Bellicus's philosophy - as advertised to other players - was one of balance. They were ambitious, interested in PvP and aiming to be well-known and respected on their server. At the same time, they accepted that members "have lives" and did not demand that game commitments take priority as many ‘hardcore’ guilds did.

The original leader of Bellicus was a male player known as Elleon. Elleon had been a guild leader in a number of other games, starting, he told me, when he was about fifteen years old. In TERA he planned to run a guild from the game's launch, getting in early and hopefully building on past experience to become a major player on his server. Unfortunately, TERA's closed beta testing period coincided with an exam period in his offline life; it was important that Bellicus have a strong presence if they were to be successful, but exams had to take priority. Elleon's good friend Jelena, who had helped him in running a guild in the past and planned to move to TERA with him, volunteered to handle guild organisation during this time. While Elleon was often absent from the game by necessity, Jelena was frequently online, vocal and helpful in chat. She wrote content for a guild website paid for by Elleon, handled recruitment and introduced new members. It was in response to one of her advertisements that I joined Bellicus in April, two weeks before the official release.

As well as being the main recruiter, Jelena put a lot of work into making the guild a community. She researched other guilds to evaluate potential allies or rivals, and collected useful resources in the form of guides or videos that other players had made, sharing these in the guild's forums. Around the open beta and early access periods she organised events to bring members together and encourage co-operation and involvement. The night before the open beta test began, Jelena organised an 'icebreaker' on Mumble (the guild's external voice chat platform) where guild members could meet and get to know each other. This included activities and games 
for which Jelena organised attendees into groups, gave directions, and kept scores. At its busiest point this event drew thirty people at once (many guild members dropped in and out around offline commitments), and after Jelena's planned activities were over many people stayed online to chat about TERA. Members discussed their plans for the game, debated the merits of character classes, and asked questions of others who had played more of the game. At one point the conversation turned to me, my research, and the guild's feelings about my presence - and I learned that they were more concerned about the combat consequences of the lag I would experience playing from New Zealand than about my research ethics. The icebreaker activity was effective at solidifying the beginnings of a sense of fellowship among Bellicus's members. The use of voice chat in particular, as a 'richer' communication medium than text, helped us to connect with each other as people (not characters) in a more immediate, less mediated way (cf. Williams et al. 2007).

For many guild members, Jelena's role as co-ordinator in this event solidified her authority as a guild leader. It was she who was seen to put in the time and effort and whose participation was most visible, and this earned respect (cf. Butler et al. 2002). As she continued to be the main organiser and Elleon remained in the background, some members questioned Elleon's position as the official leader. As he later recounted it:

"[Jelena] said, you know a lot of these people are like, 'why is Elleon the leader, he doesn't do anything, you're doing everything' ... you know, said that people were kind of questioning it, and she was like 'you know if we become co-leaders, we can share, then everybody will be happy."

Elleon agreed to her suggestion of co-leadership as a "practical" solution. In fact, this arrangement was complicated somewhat by the way that guilds were implemented in TERA. Within the game environment guild members could be separated into a number of customisable ranks, each of which had certain capabilities and privileges defined by the leader. However, the system assumed a single leader for each guild, and only one player could hold the rank of Guild Master (GM) at a time. This was referred to as "sitting in the GM's chair", a spatial metaphor which suggests the exclusivity of a royal throne. It is also similar to the 'administrator' role common in 
computer-based networks generally, as most such software identifies one 'owner' of the network who is clearly identified and has centralised control over all settings (Butler et al. 2002:8). The Guild Master had full control over the guild's status and settings within the game, including some privileges which could not be shared with other ranks. Jelena and Elleon agreed to 'pass lead' between them, meaning that they would take turns holding over this official position in the game, handing it over when they logged out or when the other wanted a turn. No formal schedule was arranged.

\section{Tensions and Teamwork}

"Now that I'm the guild leader, I can enact my plan to become supreme ruler." “Oh, don't be lame. So lame."

(Elleon and Jelena, guild chat)

The fact that the game only allowed a single person at the highest rank of leadership made it difficult for Elleon and Jelena's arrangement to be equal in practice. Through TERA's official launch and first few weeks Jelena continued to be the most prominent and vocal leader, and she was the one to officially found the guild ingame. Between his own limited time and Jelena settling into the role of leader, Elleon rarely had an opportunity to hold the Guild Master position for a significant amount of time, which compounded his problem of seeming uninvolved and unknown to his members. The two leaders had different leadership styles and valued different things in their game community. Jelena did a lot of social work, of the kind associated with female gender roles (Williams et al 2006:348, Yee 2006b) - participating in discussions, diffusing tensions, arranging activities and sharing information and resources about the game. She made an effort to be friendly with all members, and in turn they brought their problems and questions to her. Even when other guild members organised events people defaulted to Jelena when they were uncertain or unaware of the organiser:

\footnotetext{
Mukai: jelena

Mukai: is the bam thing

Mukai: we going to velika

Mukai: or alma?
} 
Kazadian: yeah meet at the statue and we will try to start at 5:30pst 8:30 est Mukai: kk

Jelena: kazadians hosting so listen to him ${ }^{\wedge}{ }^{\wedge}$

By contrast, Elleon was less inclined to organise extra activities outside his pursuits in the game. He paid for guild resources like the website, but contributed little content himself. "I take people through instances, I help level them, do quests," he told me. “Just because I don't do events, doesn't mean I don't actually lead.” For him the guild existed to support people in playing TERA, and sharing in gameplay was how members would get to know each other and bond - the gameplay was, after all, why they were in this world. The language used in early guild advertisements reflected his approach, emphasising freedom and choice. As he put it, "I honestly believe that, when you play a game, and you join a guild, and they want you to do all these things, you're paying a monthly fee to jump through hoops for someone else. That's not enjoying the game." Scholarly work has similarly noted that when participation in an online game becomes an obligation, it becomes more like work than play (Graham 2010, Yee 2006a). Jelena and Elleon's different priorities demonstrate that within the free and fluid kinds of relationships that the game fostered (some fleeting, some longer term), individuals could have quite different expectations about exactly what commitment was required. As a player, finding a guild was largely a matter of matching these expectations to one's own.

Despite their differences, the two co-leaders seemed to handle the power-sharing relatively well. Bellicus quickly reached and surpassed one hundred members and there were usually twenty or more online at peak times, creating a reliably social atmosphere in chat even as people came and went. We participated in guild warfare and developed relationships and rivalries with some of our server's leading guilds. Sometimes drama and scandal worked in our favour, as when two members of Bellicus's main rival guild so disapproved of their own guildmates' conduct during a guild war with us that they defected and joined Bellicus instead. The first major hurdle came when a high-level member named Sikander was 'kicked' (that is, removed or banished) from the guild. Elleon openly explained to guildmates who asked that Sikander's bad behaviour had driven a recent recruit and his friends to leave the guild. "His verbal abuse is not tolerated," Elleon firmly stated. "He has an 
attitude problem ... THAT is not the kind of person we want in Bellicus.” In response, a number of Sikander's friends (many of whom were said to know him offline) removed themselves from the guild, apparently in a gesture of solidarity.

For guild members logging in to the game after the event, this looked very bad - a large group of experienced, high-level characters leaving all at once suggested something had made Bellicus no longer an appealing choice of guild for them. However, on this occasion Elleon and Jelena presented a united front. Elleon did most of the explaining himself, but both stood by the decision despite its consequences. "He got three warnings before then as well," Jelena told members. "Sikander broke the rules a few too many times, that's all." Although the departure of eight members in total over this affair caused a small upset, within days the guild had settled back down and moved on. The co-leaders had weathered their first minor scandal and maintained order and morale for Bellicus.

As Williams et al. suggest, guildmates were like co-workers: once they were no longer there on an everyday basis, they were easily forgotten (2006:353). As players moved freely between different games, they could also move freely between groups in a particular game. Steinkuehler and Williams (2006) suggest that although close relationships can form in online games, they are better for fostering wide networks of loose connections, 'bridging' capital (cf. Putnam 2000); it takes time for any of these to deepen. Players' relationships with each other were based on short-term thinking and were quite selfish. A minor slight could lead to the complete abandonment of a social group, but TERA provided many more which a player could easily attach themselves to. Losing Sikander and his friends was a scandal for a day or so, but for most guild members those leaving were only vaguely familiar names, and nothing changed dramatically with their absence. This approach to voluntary social associations is consistent with the way that players were mobile agents in the 'ludisphere' of games described in chapter three; with the same mentality, they shifted between guilds and friendships without commitment despite often passionate participation while they were there. 


\section{Fission}

"I wanted to sit on the GM chair so people know that I exist. But the thing is - it's true. You know, nobody ever came to me about leadership things, everybody would go to Jelena."

[Elleon, interview]

The first signs of tension between the leaders arose when the role of Guild Master was complicated by the introduction of 'vanarchy', the player-based political system which was one of TERA's most unique features. On each server the game world and map was divided into fifteen provinces, each of which was to be ruled by a 'vanarch' who could change certain rules and affordances within his or her territory (for example setting sales tax rates and making services available). Vanarchs were elected (initially) by vote, and the provinces were divided into three 'continents' with every player able to vote once in each continent. To run for the office of vanarch, a player had to be a Guild Master and able to pay the registration cost, consisting of both gold and medals earned by guild members who completed certain quests. In effect, only leaders of large guilds could gather the resources to run, and successfully administering a province required the continual provision of similar resources. Elections were to be held every month, with the first beginning at the end of May. Bellicus would, of course, put forth a candidate, and Elleon expressed his desire to be the guild's first vanarch. Already busy, Jelena readily agreed to let him take that role, along with chief responsibility for the electoral campaign.

Because a vanarch candidate had to be a Guild Master to register, Elleon gained formal leadership of the guild in-game. Furthermore, once he had registered as a vanarch candidate it became impossible for him to give the rank to another person until after the election. For the three weeks between registration and the end of the voting phase, Elleon alone could be Guild Master. Elleon appreciated this opportunity to have more control and be more visible to his members, and initially Jelena was glad to have some of the work lifted from her shoulders. However, after running guild affairs and publicity for so long, it was difficult for her to step back and instead let Elleon run things in his quite different style. With each player having three votes for vanarchs, guild members would be expected to vote for their leader in 
one continent, but the two other votes provided an opportunity for voting alliances where leaders pledged their guilds' votes to support an ally on another continent. Elleon focused on forging such alliances through private conversation with other Guild Masters, rather than on publicity.

Jelena openly criticised Elleon's actions for the first time during the early campaign period, implying that he was not doing enough to promote himself as a candidate. Other leaders were putting together promotional videos and participating in forumbased debates, activities which Jelena thought were important and urged Elleon to do as well. When he made a spelling error in a post on the guild's own (private) forums, she corrected him publically and with a critical tone:

“The capital city of southern Shara is called Allemantheia. Make sure you get the names right when in contact with other guilds or writing any sort of campaign information. People may not want to vote for someone who gets the names in the game incorrect."

Elleon responded with a cold, "Jelena I know what the areas are called.”

The tension between the two leaders was aggravated by a death - Elleon's. Bellicus were in the midst of a guild war with a rival guild, and Elleon was playing in a heavily populated area where enemy players could easily find him. An enemy ambushed and killed him, earning their guild ten points for slaying an enemy Guild Master and effectively costing Bellicus the contest. Although Elleon apologised for letting it happen, Jelena and some others felt that he had been irresponsible for leaving himself so vulnerable during a war. Jelena messaged him privately and suggested he make her Guild Master instead, as "I'm just sitting in town because I'm [level] 6o, all I do is queue for dungeons." As this was during the campaign period, the game would not allow him to, and he told her so. To his dismay, Jelena asked for proof (a screenshot). Elleon saw this as displaying a "lack of trust" which was wholly inappropriate in a co-leader and, worse, a friend. "I thought that was one of the stupidest things anyone's ever asked me for," he told me. "I was just disappointed." He sent the screenshot resentfully. 
The final spark which brought the budding conflict to a head was the departure of a member called Kaidun. Kaidun had been with the guild since the beta tests - longer than I had - and he was friendly with Jelena but disliked Elleon for what he saw as the exploitation of Jelena's work. He grew bored of TERA, and in late May he decided to quit the game. As a parting shot, given that he no longer had any particular investment in the guild, he used his officer rank privileges (granted recently at Jelena's suggestion) to access the guild bank and take the majority of the gold and valuable items. Then he left the guild. It took some time for other members to notice, but when they did Elleon was furious; we had been robbed. Automatically recorded logs clearly showed that Kaidun was the culprit. Knowing that she had been on good terms with him, Elleon urged Jelena to confront Kaidun about what had happened, preferably getting the guild's wealth returned. Much less distressed, Jelena brushed off his urgency and told him that they should be able to get the gold and items returned by contacting customer support and laying a complaint.

Recent stresses and frustrations with the guild had led Jelena to consider leaving Bellicus, which Elleon was at least partially aware of. Jelena felt that Elleon was not doing his fair share of the work in the guild, and that when he did act it wasn't in accordance with the rules that they had agreed upon together. In turn Elleon had begun to express his opinion that she was power-hungry and trying to run the guild by herself, preferring to cut him out of things. Each blamed the other for not being willing to share power fairly. When Jelena finally became fed up and prepared to leave, she decided she would speak with other members individually, "because we have very different leadership styles and some people might like Elleon's and some people might like mine. I thought it was fair to give them the choice." She was no doubt aware that her greater presence in the community would win her sympathy, and many did choose to leave with her. Because she had these conversations through private channels, one-on-one in text or voice, all Elleon knew was that a large number of people suddenly left the guild in succession. This, combined with Kaidun's theft (which Elleon felt that Jelena was suspiciously unsurprised by) led him to conclude that she was 'poaching' members to start her own guild, taking advantage of the people and resources Bellicus had accumulated. 
In response, Elleon (still entrenched as Guild Master with all the rank's privileges) kicked Jelena out of the guild.

\section{After the Split}

“Originally we agreed to be co-lead and pass back and forth, but...being co-leaders means you split the work, and you split the responsibility, and he wasn't splitting the responsibility with me."

[Jelena, interview]

Both parties were upset with each other, each feeling hurt and betrayed by a friend who should have been supportive and trustworthy. Elleon believed that Jelena had conspired against him, stolen his guild, and undermined their supposed coleadership in order to have everything for herself - "because even if she was a coleader, or whatever, this started out as mine." Jelena believed that Elleon had wanted to enjoy power without contributing to the work, and had not shown her any of the respect due to a co-leader or a friend. "People...were telling me what Elleon was saying in Bellicus guild chat, and of course he was blaming the entire thing on me," she told me. For most other guild members, the whole affair was primarily confusing. The many (myself included) who did not happen to be online when Jelena was kicked logged on hours or days later, often with no warning, to find the guild roster severely reduced and familiar names missing. In some cases people had difficulty finding out what had happened, and the leaders (dealing simultaneously with the end of their friendship) were not always amenable to repeating their version of the story for every person who logged on. Members faced a choice: stay with Elleon and the original guild, leave to rejoin Jelena, or a third option - just leave altogether, and find a new guild. This was not uncommon, especially among those who did not expect either group to truly recover from the upheaval and return to the business of the game.

In the days immediately following the split, there was some hostility between the leaders; Bellicus' recruitment advertisements had to be revised, and spiteful comments and accusations were occasionally made quite publicly in the TERA forums. Yet just as when Sikander and his friends had left, both parties soon made an effort to move on. "What I did, I did for the good of the guild," Elleon repeatedly 
reminded his followers. Jelena formed a new guild of her own, called 'Renaissance', and encouraged those who followed her from Bellicus to forget about their old guild and focus on starting afresh. "It's pretty nice," she told me of her new guild, "no stress, no drama, it's like a vacation." Meanwhile Elleon assured his remaining members that "Jelena's departure will not affect the guild in any way," and also encouraged his members to leave this affair behind and look to the guild's future with the vanarch elections.

Certain level-headed advocates on both sides of the conflict quickly began posting soothing messages on the TERA forums and both guilds' websites, which insisted that they bore no ill will toward the other group despite the scandal and that the split could proceed amicably. Sure enough, within a week the two guilds Bellicus (led by Elleon) and Renaissance (led by Jelena) began to settle as self-contained groups which each made a point of ignoring the other's existence, at least on the face of things. They continued to play on the same server, but did not engage in guild warfare with each other or show other signs of carrying a grudge into their gameplay. Both leaders approved of my decision to remain as a member of Bellicus, although I would stay in touch with Jelena and Renaissance, eventually creating a second character to allow me to observe and participate in both guilds' chat (though not at the same time).

With Jelena and her followers gone, the change of atmosphere in Bellicus' chat channels was immediately noticeable. There was significantly less idle banter and discussion. Where twenty or more members could be expected to appear online during peak times before the split, afterwards the average was closer to four, usually the same four dedicated people each day. Aware of the work Jelena had done to encourage and mediate socialising in guild chat channels, Elleon made a deliberate effort to be present, friendly and vocal as much as possible in the wake of her departure. The vanarch elections were imminent and he needed to create a sense of normalcy to prevent more members from leaving. He began recruiting new members to replace recent losses, but he could not be online all of the time and without him there was little interaction between guild members. Many new recruits, getting nothing out of their membership, silently left just days after joining. Members may have operated as individual agents, often not even playing co-operatively, but in the 
wake of the guild's fission it was evident that leadership has played an important role in the guild's cohesion. The deathly silence in guild chat did not bode well for the guild's future; although a number of capable players remained, most of the socially motivated members had followed Jelena.

When the elections concluded and Elleon failed to secure a province, he began to withdraw from the guild. People continued to leave, newcomers were introduced less frequently, and when Elleon eventually removed inactive characters the guild roster was reduced from 115 to only 33 members. Although Elleon stuck by his statement that "if I'm the last person in the guild then so be it...a captain goes down with his ship," he seemed to have largely given up on the guild after the election. He was rarely online, and it became common to find no Bellicus members logged in even during peak times.

Renaissance, Jelena's new guild, fared a little better. With perhaps as many as thirty members joining her from Bellicus, she began recruiting successfully and quickly brought membership up to and beyond 70 people. The guild's chat channel was reasonably active, with ex-Bellicus members carrying the majority of the conversation. During peak play times, an average of six Renaissance members could be expected to be online, in contrast to Bellicus's initial four (which soon dropped to only one or two, then none by mid-June), and there was some variation in who was present. Nonetheless this was nowhere near the twenty or more concurrent players that the original had guild boasted in its heyday. What Renaissance lacked was the ambition that had driven Bellicus at its inception. The guild was rarely involved in guild versus guild warfare and did not put forward a candidate for vanarch; as one member put it, "no one seems interested in progressing as a guild, everyone was doing their own thing. I got bored just sitting around." This was in part symptomatic of the stagnation of the server community in general as players grew bored of the game (see chapter three), which provided little incentive to drive such ambition. The few scheduled events that Jelena initially tried to organise never got off the ground, and a month after the split from Bellicus even she was rarely participating in guild activities. Renaissance became a loosely-connected group of people who sometimes played together but did not have a strong shared identity as a guild. 


\section{Transient Relationships}

"We can get new members, easily; I care about you guys, but I don't care about these people leaving, they're not the kind of people we want anyway." (Elleon, guild voice chat)

Both of these guilds can be said to have ultimately failed in their original goal of becoming prominent and respected on their server, despite a very strong start before and around TERA's release. Bellicus, the original guild, caught the eye of other PvP contenders during beta testing and participated often in guild vs. guild warfare against notable opponents, making their name known and establishing some tentative alliances and rivalries. The large number of members participating in beta tests allowed the guild to be ready for serious competition relatively quickly, among the fastest although others were even quicker. At the same time, social organisation and Jelena's focus on building a community helped to solidify a shared identity and a sense of pride, motivating players to contribute to their guild and to help each other succeed. Although not one of the most obvious forerunners on the server, Bellicus was active and recognised, as indicated by the fact that Elleon was solicited for vanarch votes by major guilds. Despite these advantages, both Bellicus and its offshoot Renaissance were inactive within three months of TERA's release.

The failure of the guild is not exceptional: according to Williams et al. (2006:349) $21 \%$ of guilds founded in World of Warcraft were disbanded within a month. Bellicus went through what felt (from the inside) like rapid extremes, from extremely active and motivated to a divided and inactive group in a few short weeks.

Ducheneaut et al. note that "guild membership encourages players to play more," meaning guilds benefit the game overall, but "growing a guild to a significant size and sustaining it over time is a difficult task" (2006:8). Bellicus's early success came alongside the strongest period for the game community as a whole. Purchasing TERA gave players a month of free play time before subscription fees were required, and fans were eager to push through to advanced areas which had been unavailable in the beta events. PvP drove players to rush toward the maximum level, encouraged by 
encounters with other guilds ${ }^{14}$ which kept the game interesting, varied, and significant for players. When players later began to lose interest in TERA, maintaining a guild within the game began to require more effort.

Debeauvais et al. state that "guilds increase player commitment because they add new motivations for their members" (2011:185), and a few months into TERA's release players who had reached 'endgame' were in dire need of a reason to stay. A strong guild might have kept them engaged - but those who were losing interest in the game were less likely to invest in a guild community that was tied to it. Members had to be willing to log in at all before they had the chance to be social participants in a guild, and if they lost interest in TERA's gameplay they could simply disappear by never choosing to visit the game world. TERA was always inhabited partially, and events there were not connected to other friends or communities in one's geographical location. In this world presence was not a default state, but rather something which required a deliberate choice and effort to convey. Without this effort, a person could suddenly cease to exist as far as the game was concerned.

A guild was a voluntary association, and while in some games they are all but essential in high level play (e.g. Ducheneaut et al. 2006; Jakobsson 2006; Johnson et al. 2009; Taylor 2006b), in TERA the prevalence of the automatic instance matcher meant less emphasis on guilds, outside of the soon unpopular vanarch system. Even $\mathrm{PvP}$ could be undertaken without a guild if one were willing to be a lone bandit, but with over a thousand guilds to choose from just on Sienna Canyon server it was always easy to find a new one. Although a player might form strong friendships or emotional attachments that made the decision to leave their guild a weighty one, mechanically it was a simple matter of clicking a button to leave, and clicking again to apply elsewhere. Guilds might have application forms for new members, but few had exceedingly rigorous screening processes; one could be in a new guild by the time one logged off that night. The utility of a guild, at least, was easily replaced, and with guilds advertising on the forums and recruiting in the game it was easy to explore new options.

\footnotetext{
${ }^{14}$ For example, at a guild gathering one day a small group from a rival guild tried to ambush us, only to find we outnumbered them significantly more than they had expected. We turned the tables and chased them back into a safe zone, sparking a lasting rivalry between our two groups.
} 
Bellicus's rapid rise and fall illustrates how on both the individual and group scales, most in-game relationships were impulsive and transient - entered into easily but cut off just as easily. When Sikander was cast out, five other members left to follow him; Sikander was kicked because he offended Dulari, who had only recently left his previous guild to join us. Dulari then left Bellicus in turn over this affair. All of these players were involved in guild chat and activities, but abandoned their guilds with apparent ease - in a manner that paralleled their approach to new games. In this sense the social world of TERA was also treated as a marketplace, where players could pick and choose from among the groups on offer. The mentality of customers in the ludisphere, experienced and knowledgeable but fickle in their desire to be entertained in the here and now, played a part in people's interaction with each other as well as with companies. If one's immediate purposes were not helped by a particular affiliation, one could shift to something more useful or enjoyable with ease.

Of course serious personal conflicts still occurred, and occasions such as Elleon and Jelena's falling out saw social drama played out in full within the game. Nonetheless, the speed with which normalcy could be restored just by putting a conflict out of people's minds was notable. Change, even sudden and tumultuous change, was part of life in TERA, an environment which was constantly being tweaked, upgraded and adapted in reaction to how players played. The players I knew were used to this, not only in game updates but in their online social lives and in their own choices as they dabbled in different games, never quite satisfied with what was on offer. Players thrived through their identity as discerning, impatient customers whose attention had to be courted, and so it made sense that their in-game friendships reflected this identity. The following chapters will explore this attitude further.

\section{Conclusion}

Bellicus began as a highly organised and ambitious group of experienced players who established themselves on their server quickly, but a combination of differences in leadership style and personal tensions, exacerbated by certain guild mechanics present in TERA, eventually lead to the group's dissolution. The decline of the guild happened alongside and within the decline that TERA as a whole was going through 
(as recounted in chapter three), and these two 'life cycles' fed back into each other, with players who were less interested in TERA generally being less inclined to put effort into getting their guild back on its feet. Players were fickle and focused on the immediate moment; in-game relationships could be friendly, but were often shallow and easily forgotten if one moved on. Little was permanent, choices were ongoing and could be changed, and impulsive decisions often ruled where the software made it easy to quit a social group entirely in a fit of pique. If "to be an agent means to be capable of exerting some degree of control over the social relations in which one is enmeshed" (Sewell 1992:20), players enjoyed considerable agency in their relationships with each other, with the systems of the game supporting their freedom of movement. In choosing friends and allies they applied the same marketplace logic that they used to evaluate which games to play and for how long. In the following chapters I will argue that players strongly identified as consumers, and that their position as mobile, choosing agents was part of their wider relationship to the game and its developers. 


\section{Fairness and Legitimacy}

TERA players were well aware that their game world was designed, with developers controlling most variables in gameplay, interface, and the world itself. As they represented the world's designers, En Masse could be blamed when things were not to players' liking, and they were expected to change the game accordingly. As a game and a service for which players paid a fee, TERA (unlike 'real' life) was expected to be fair - all players were to have an equal chance to participate and to be successful. At the same time, the nature of the game was that not everyone could be equal, or there would be no competition. The ideal was for distinctions to be made on the basis of skill (including both strategy and reflexes), with the game elements as relatively neutral tools at players' disposal and the player themselves as the variable that mattered in a contest. This chapter will demonstrate how the ideal of a skill-based meritocracy was an underlying basis for players' behaviour and demands throughout the game. It can be contrasted with the complex realities of the western democratic capitalist societies from which TERA players overwhelmingly originated, but it also extends and adapts the ideals of such societies into a new scenario.

\section{Expectations of Fairness: Balance}

“Ganking a lone mystic, shame on you"

"A mystic shouldn't be complaining. Op class."

(Area chat)

Accessing TERA as customers of En Masse entertainment, players brought with them certain expectations about how the game would be built. One of the most important was the expectation that players would have equal opportunities to succeed in the game's objectives - that the world would be fair. Although it is a cliché in the offline world that life is not fair, MMORPG players entered game worlds not as natural inhabitants but as paying customers, and as such expected to receive neither more nor less than any other paying customer. Players were well aware that the creators of the game had the ability to control most factors which might allow anyone to gain a unique advantage (outside of player interactions with each other), and they expected 
them to manage these as part of the service that En Masse provided. At the most basic and most important level, this was phrased in terms of 'game balance'.

Players could choose to create their characters as one of eight classes, each of which not only fell into one of the three main roles (tank/heal/DPS15) but also had distinctive mechanics and a distinctive style of play that made it different to the others. For example the slayer, a sword-wielding damage-dealer, did not store and spend 'mana' like a spell-caster, but instead earned it through striking enemies. Slayers thus required a warm-up to use their more powerful skills, while my priest character was most capable early in a fight before using up her mana. In choosing a class, players were choosing their role in combat, the kind of weapons and appearance they wanted for their character, and the specific techniques they would attempt to master. With this choice came an assumption (carried over from other MMORPGs and wider role-playing game contexts) that no class was innately better or worse than any other - rather, they each provided different ways of contributing in a co-operative group. As a further example, in TERA priests had more direct healing abilities, but mystics had a more diverse range of support skills. Players compared the relative benefits of these two healer classes frequently and at great length, with plenty of advocates for both sides. It was assumed that no matter which class you played, if you were good at using the resources available to that class, you could be successful.

TERA was, essentially, assumed by its players to be a 'meritocracy', to use the term coined by Michael Young (1958). Where Young's fictional meritocracy is based on merit as "intelligence + effort", TERA players recognised merit as skill. Individuals achieved success, wealth and renown through their own skill at the game. Caillois (1961) refers to merit as the quality tested in games of 'agôn' - competitions - and emphasises that a game must create an artificial state of equality between contestants in order to test their ability in one specific area. In TERA a class or race choice which had a bigger impact on their success or failure than their personal ability did was considered 'unbalanced' and required fixing - and En Masse Entertainment, as 'service providers', were held responsible for maintaining this artificial equality. A character was the avatar through which one was represented in

\footnotetext{
${ }^{15}$ That is defender, healer and damage-dealer (DPS stood for damage-per-second).
} 
the world and could contribute to group play, and class determined and constrained the actions that a character could take in combat (the main in-game activity). Unlike members of offline societies who are born into a specific social context that they do not choose - including a family, ethnicity and social class - TERA players chose their character's class, but they also held En Masse accountable for ensuring "equality of opportunity" (Young 1958) no matter what choice they made. From that equal starting point, it was assumed that those worthy of special note or reward would stand out when their skill was fairly tested.

Whether or not TERA achieved the intended equality of opportunity was inevitably contested - the usefulness of a class was a subjective judgement. Players would complain that a class was "OP" ('over-powered') if they were defeated and felt that they hadn't had a fair chance to win, deflecting blame away from themselves with statements like "slayers are good even if played poorly", or "slayers are braindead16 and OP. Common knowledge." Alternatively, some focused on their own class's shortcomings, claiming that unbalanced classes made it impossible for them to succeed in certain situations regardless of their own skill. The structure of the game always constrained player actions, but it could be perceived as undermining one's efforts when it should have been enabling fairer contests. When players complained that their class was under-powered the most common responses were either commiseration from others who felt the same, or (more often) an insistence that the player simply was not skilled enough. For example, "warriors are bad unless played well" was a common sentiment, and one player accused "[previous commenter] is just pissed because he sucks too much to play a warrior." In these cases, players defended En Masse's balancing choices and blamed player error for any apparent discrepancies - but only when it was other players who were the inadequate ones.

One player expressed his opinion particularly clearly in response to a discussion about what needed to be re-balanced:

\footnotetext{
${ }^{16}$ In this case referring to the fact that while most classes in TERA were said to require careful attention to positioning, distance, dodging and the environment, the mechanics for slayers were often said to be simple enough that charging in and attacking was sufficient to get through a fight.
} 
"No class is OP. Stop trying to get them nerfed because you fail. Tera's got a good balance going \& if they nerf one class, the rest of us will follow to match the nerf made. Keep that, "flavour of the month" shit at WoW..."

'Nerf' was the term used to describe an official change to the game that made something less effective than it had previously been. Major mechanics or specific skills could be nerfed in any update - an example of the power developers had over their online game worlds. This could be upsetting for players who had finely tuned their characters to maximum advantage under one set of rules only to have the rules changed from under them. The player quoted above draws upon his experience of another game, World of Warcraft, to extrapolate how small 'corrections' can lead to changes for the worse in the game overall. Aware of the frequency with which changes and updates could be made in MMORPGs, players planned for and tried to anticipate them, as with the player who told me, "I was going to be a slayer, but everyone was going to be a slayer. I was going to be a warrior, but they're probably going to get nerfed.”

Online games are distinctive as perpetual works in progress rather than completed artefacts, part of a process of game design in which players and developers maintain a relationship (see Stenros and Sotamaa 2009). Because TERA was updated ('patched') regularly, En Masse had the ability to make corrections where players perceived mistakes. It was expected that any issues which came to light after the initial launch would be addressed as part of the continuing service for which players paid subscription fees. The structure of the game and its rules may have been set and unyielding at any given time, but there was always the potential for adjustment (and appeasement) in the future. While some players felt that balance issues constituted deep flaws in TERA, many more were satisfied with "waiting for new stuff, updates and putting faith into EnMasse in [the] hope that they'll make things right eventually," as a guildmate told me. The perfect balance would likely never be achieved, as players continually reacted to each update and new problems emerged, but the cyclic process of feedback and adjustment continued to strive for Caillois's artificially levelled playing field. In this we see the 'duality' of structure and agency recursively constituting each other (Giddens 1984), but where the process is usually seen as a slow and subtle one whose effects may not be seen for some time (Ortner 
2006:9), in TERA it occurred through conscious direction, with updates cataloguing the specific changes made to the software at each point in time.

\section{Game Balance and Social Power: The Lancer}

"Being a lancer makes me feel like a king."

"Go do your real life stuff meany. You never queue with us since you hit 6o." (Guild chat)

In chapter three I discussed the bottleneck experienced by players using TERA's automatic instance matcher to find a group for the instanced 'dungeons'. Players I encountered unanimously attributed this to the fact that lancers were the only character class who could fill the 'tank' role in group play, and so no group could proceed without first finding a lancer. This made one of the eight character classes integral to the main occupation of level 60 characters, and all players of other classes became dependent on the relatively few lancers. As well as creating the practical problems described earlier, this gave lancer players a significant amount of power over others within the game. It took a long time for the instance matcher to put together a group, and if the lancer left, the rest of the group would have to return to the queue and wait all over again.

A striking effect of this inequality among classes was the way that players of another class, the berserker, began to be excluded from instances. TERA classes were divided into three categories by the armour that they wore: cloth for priests, mystics and sorcerers; leather for archers, warriors and slayers; plate for lancers and berserkers. This placed berserkers in competition with lancers for any rare plate armour that dropped as a reward. Normally, any group member who wanted to make a claim on an item would 'roll' for it, and the game client made a virtual dice roll for each claimant and bestowed the item on the player who 'rolled' highest. Once lancers became valuable enough that a group could not proceed without them, they no longer had to submit to this egalitarian system. Lancers could simply insist that any berserker was kicked from the group, and group leaders could not afford the risk that the lancer would leave if his or her demands were not met. By effectively disallowing berserkers, lancers could ensure they faced no competition for the armour items they 
wanted. A feature of the way the game structured classes and class balance resulted in a form of discrimination.

The consensus in public chat seemed to be that berserkers could overcome this systematic disadvantage if they were members of a guild, whose lancers would presumably be willing to play alongside them and help them get the rare armour that they needed. Despite this, I saw the class conflict played out within my own guild, notably between two of the early, established members who were among the first to reach level 60:

Deigan: $\mathrm{i}$ have this really weird feeling that if $\mathrm{i}$ queue for a 60 dungeon $\mathrm{i}$ will get kicked as soon as the lancer sees me

Barlyas: thats what i do

Deigan: ppl17 just be dicks need to learn how to share :D

Deigan: its only a 50/50 chance

And on another occasion:

Deigan: when I get 2 more $\operatorname{lvls}^{18} \mathrm{u}$ should run some [dungeons] with me :D Barlyas: you're a berserker... lancers don't like berserkers in group :)

Deigan: i am the only 60 zerker in the guild and i can't even que with a tank

Deigan: kinda sad

Deigan: makes me angry really

In both of these exchanges smiley face icons are used to soften what could otherwise be rather harsh or confrontational remarks, but Deigan's real frustration is evident. Guild members were supposed to be supportive and work together towards goals, especially when members needed equipment to progress. In this case, the class division was prioritised over this relationship; Barlyas chose to reiterate his social power as a lancer even among guildmates. Players may have been able to choose their character class, but when making that choice they were not always aware of the full implications it would have. This kind of imbalance could emerge unexpectedly as a game (or server) community developed over time; those who created their

\footnotetext{
${ }^{17}$ Abbreviation for 'people'.

${ }^{18}$ Abbreviation for 'levels'.
} 
characters right at the game's release, as most members of Bellicus did, had the least information to go on. Character class was in some ways like caste, in that it was a permanent feature of the character ${ }^{19}$ and determined their role within the game's society - although that role was more rigidly enforced than in the physical world. A player could later create a new character using their expanded knowledge of that society, but would have to put in many hours of work re-treading the same ground to reach the equivalent status of their original character. Additionally, many players told me that they chose their class because they enjoyed its distinctive play style. Social dynamics evidently meant that it was not always easy to progress while playing the game in the way one wanted to.

Players were very much aware of these dynamics and knew that lancers had acquired a kind of social power that was never factored into the balance of the game. They did not blame En Masse for this imbalance, as it was not strictly the game mechanics that were at fault but rather the distribution of players among combat roles. Nonetheless public chat reflected an awareness that the emergent tank problem was upsetting an ideally egalitarian system:

Leinyn: if buffing warriors as tanks puts lancer's in their place, and reduces lancer douchiness.. im all for it

Aleister: same. Zerker here, feeling a bit annoyed that I get kicked when i say i don't need the loot

Siera: Theres douches in every class. Why specifically focus on lancers? Lienyn: because being tank they generally hold the most power in the group Loxetrius: bcz ${ }^{20}$ lancers are required douches

\footnotetext{
${ }^{19}$ A character's race, gender and appearance could be changed if a player paid real money through En Masse's website, but class was too integral to a character and could not be changed.

${ }^{20}$ Abbreviation for 'because'.
} 


\section{Luck}

"In real life it's not as easy as a video game."

(Interview)

Players demanded an equal start, and a balance which ensured that any character they chose to make at least had the potential to be successful with skill and hard work. At the same time, the designers of the game had an interest in giving players something to strive for. "Scarcity is what makes the [virtual world] so fun...people seem to prefer a world with constraints to a world without them," Castronova (2001:15) reports; this is part of the game, and the more common an item becomes, the less prestige value it has for those who attain it. Here, then, there is a tension between a desire for fairness and egalitarianism and the competitive drive which modern capitalist consumption encourages. The developers of TERA, as with many other MMORPGs, used elements of luck in the game's mechanics to compromise. Where chance was involved, all players could have an equal chance to succeed, but only some players did. By taking the decision out of the hands of either the developers or other players, it could remain fair for all parties but still introduce certain inequalities into the community of players in the game world, encouraging competition. It also meant that trying again (repeatedly) might be the only way to achieve some things - which suited a company whose business model was based on players subscribing in the long term.

Two major aspects of the game (especially at higher levels) that were based heavily on chance were loot rolls and enchantment. When a rare treasure dropped and more than one character 'rolled' for it, chance determined which of the characters received the prize, with the probabilities presumed equal for each participant. Party members could choose whether to roll or not, but could not directly affect the outcome if more than one person rolled for an item. Enchanting an item could increase its strength and usefulness, but a player had to find or purchase valuable components just to make an attempt. Each attempt to enchant had a chance to fail, destroying all components except for the item that was to be enchanted. As the level of the desired enchantment increased, the probability of success in each attempt decreased, making it difficult to reach the highest levels of power. Nonetheless all players were subject to 
the same (deliberately engineered) probabilities for enchantment, and if any player achieved a level more easily than others, it was because of luck, not because of any systematic advantage. In contrast to what were perceived as balance issues, En Masse could not be held directly responsible for an individual player's luck, although the tuning of the odds could be questioned.

One player, Adean, suggested to me that the way luck and chance were implemented in video games was a large part of the appeal for some players. "You're not dealing with pure luck in most cases in real life," he told me. "Real life luck is random...and doesn't happen as often." The players I knew were overwhelmingly North American, from the United States or Canada, and would have been encultured into the idea of the 'American Dream' of social mobility. In reality, the United States at this time was a significantly less socially mobile nation than that ideal suggested, with parental income largely determining one's education prospects and future income (Blanden et al. 2005). Factors in life which were difficult to change, such as gender, ethnicity or socio-economic background, played a large part in shaping people's prospects. 'Real life luck' for Adean constituted a rare opportunity that was not determined by these factors, but by happy coincidence.

By contrast, online games presented all players with instances of luck on a more regular basis, in the form of 'drops' that were a potential reward every time an enemy was killed. The drops one received could affect a character's power, but all players could receive drops and were likely to receive some useful or valuable ones while playing. The probabilities involved in drops, loot and enchanting were, of course, carefully tuned by the designers of the game - in other words not 'random' luck. Game designers had an interest in keeping valuable items relatively scarce, but they also needed to keep players engaged in chasing after goals. If a perk was too unattainable, players might lose interest, leading to cancelled game subscriptions. The ideal was to have a desirable reward always almost within reach, a factor to which World of Warcraft's success has been attributed (the "virtual Skinner box" [Ducheneaut et al. 2006:7]). "Online games allow you to do things easier than in real life,” Adean told me. They were designed to make in-game achievements satisfying. 
The result of this was that TERA (and other online games like it) offered a world in which chance was in some way more fair than in the real world, because it was carefully controlled and not 'brute luck' (cf. Anderson 1999). Players could expect to know what the outcome of particular actions would be before the fact, and for these outcomes to be consistent between players, because they knew that there were human minds behind the design. Developers ultimately wanted to please the game's players - no overly offensive loss or disappointment could be allowed. In addition, what was attainable to any player had to be attainable to all, at least in theory, because all players paid the same fees for their participation in the game. TERA represented an idealised implementation of capitalist values: if one put in the prescribed amount of work and followed the advised strategies, one could always eventually - attain the greatest advantages in the game.

\section{The Random Number Gods}

"Seriously? No way to boost chances?"

"NOPE ENJOY ENCHANTING AND SPENDING ALL YOUR GOLD AND FAILING." (Area chat)

Not all TERA players approved of the way luck and chance were handled in the game. By August, four months into TERA's official release in North America, one of the most common complaints made in the game's forums was "RNG is killing this game." RNG, or 'random-number generator', has been a part of role-playing games since the dice rolls of Dungeons and Dragons, where it added an element of risk to any action a character tried to do. In a game like TERA where technical skill and reflexes were involved to a much greater extent, many players no longer wanted this element of uncertainty - they wanted to succeed or fail entirely on their own merits, and chance "seems an insolent and sovereign insult to merit" (Caillois 1961:17) as one's qualities have no influence over it. TERA was treated as a platform for direct contests between players (or of players against programmed challenges). One's character was not an imagined heroic persona so much as an instrumental extension of oneself that allowed action in the game world. Particularly in TERA, an action- and reflex-focused MMORPG, players wanted to be responsible for their own hits and misses rather 
than doing everything right but failing because of an ever-present, built-in probability of failure.

Once again the assumption that TERA should be a meritocratic system lay beneath anti-RNG arguments. Players need not be equal; rankings and prestige items assumed some inequality and gave players goals within a game that couldn't be definitively 'won'. However, skill was held up as the ultimate, most worthy distinction between players. Anything with a random chance element could override player skill and created distinctions which some players felt were 'unfair' (usually, it must be said, the players who were unlucky). "So the person with better gear from rng gods wins... if both equal in gear... he who gets [a random] crit $^{21}$ wins," one player complained on the game's forums. Under this system, even excellent players might be at a disadvantage because they were unable to get the strongest possible equipment, through no fault of their own - and even mediocre players could defeat those more skilled if they were luckier in enchanting or with other bonuses. Luck was not earned, and thus should not define status in a meritocracy - an argument in line with theories of 'luck egalitarianism' (c.f. Anderson 1999) which advocate for levelling or compensating for people's luck (e.g. factors of birth) and holding them responsible only for the results of their choices.

RNG elements were present throughout TERA. The damage done with a strike in combat was determined randomly within set ranges, with a chance for critical hits. Loot that was dropped by enemies in the open world or as prizes in instances was randomly determined by the game as well as randomly assigned between players who wanted it; this meant that even if an item that a player could use happened to drop in an instance, they might lose the roll for it. Enchantments had a chance to succeed or fail, and each failed attempt cost a player a large amount of in-game wealth. I would separate these uses into two categories: random numbers for combat and random numbers for progression. While chance elements in combat simply made fights less predictable, progression that was partly dependent on chance served to slow players down, something that served distinct commercial interests for the developers. Players who had already reached the highest levels of achievement in the

\footnotetext{
${ }^{21}$ A 'crit' or critical hit was a hit that dealt considerably more damage than normal. All characters had a set percentage chance to land a critical hit, which could be modified with equipment and accessories.
} 
game had no reason to keep playing. As Nardi (2010:112) points out in regard to farming in World of Warcraft, delaying tactics kept players engaged and paying subscription fees for longer before they required updates, which developers needed time and effort to create. Although this could be boring for players, Nardi interprets it as the cost necessary for players who wanted to "be marginally better...than the other players on [their] server."

It is interesting to note the conflicting ideas that players held in regard to egalitarianism in TERA. On the one hand, most recognized that for any kind of prestige items to exist, some things had to be rarer or significantly difficult to obtain. "I would rather not see thousands of players running around with full +9 gear and hundreds with full +12 . When I see someone with 3-4 [pieces of] +12 I know they put some work into it," one player posted. Making high level enchantments easy to obtain might level the playing field in PvP, but it would de-value them and negate their prestige value (which is a major motivating factor in virtual worlds; "since [virtual worlds] are inherently social, the achievements are relative" [Castronova 2001:15]). On the other hand, many who participated in endgame activities, especially $\mathrm{PvP}$, were frustrated by the fact that not having these highest advancements put them at a disadvantage against others who did. Bonuses such as high-level enchantments were not seen as optional for these players if their opponents could be expected to have them. Additionally, some players reported being kicked from groups with strangers because their enchantment levels were not considered high enough. In these cases, it seemed like the random chance elements were delaying access to not an optional prestige item, but a necessary component of character development - and from this perspective it was viewed as unfair because it was a barrier to progress which players could not overcome through their own effort.

TERA players wanted to be in control. When they fought, they wanted to know that their skill was being tested, and they were responsible for the outcome of the contest. If some aspect of that contest was not fair - if the contestants were not kept artificially equal in every respect but skill, as Caillois argues is necessary for games of competition - the result was inconclusive, and players need not accept that their skill was really the key variable if they lost (hence protests about 'balance'). When players dedicated time and effort to the game, they wanted to be able to see the results of 
that effort and have them be consistent. Chance-based progress took it all out of their hands. On TERA's forums one player complained of enchanting, "I myself don't feel like I'm rewarded or given the proper return for what effort I try to put in and instead it becomes a matter of sheer luck and my luck has been very bad for 2 weeks." Caillois (1961) suggests that submitting our fate to forces outside our control is the source of enjoyment in games of chance, but TERA players did not seem to enjoy what they saw as not a voluntary surrender to chance, but being forced to surrender in order to progress.

\section{Legitimate and Illegitimate Killing}

"It's fine to rage about 3 vs 1 ganks and 2 vs 1 ganks, pvp server or not...just because pvp happened doesn't mean it was fair pvp."

(Area chat)

Another area where the concept of fairness was strongly contested was PvP, or player versus player combat. I played TERA on a PvP server which allowed players to kill other players' characters in most open world zones, without warning or consent (as opposed to duels and group 'deathmatches', which required consent from all parties). Playing on a PvP server was a choice: numerous PvE servers were available, offering essentially the same game without the risk of being attacked without warning. Consequently it was common for complaints about attacks to be met with "then don't roll pvp," that is, don't play on such a server if you don't want to accept the risk. Nonetheless, what constituted good or 'fair' PvP was disputed by many players and publicly-voiced complaints were common.

The opposite of fair or honourable PvP was widely referred to as 'ganking'. Deigan, a guildmate, defined the term for me:

Deigan: gank = someone jumps on $\mathrm{u}$ when u're not prepared

Deigan: most of the time, it's many people jumping on 1 person

Ganking is thus in conflict with the ideal that success in TERA was legitimately earned through skill. While good PvP involved two relatively evenly matched participants pitted against each other, ganking often resulted in a very quick defeat 
for the target due to large level disparities or being outnumbered. This required little skill and was not an honourable victory, nor did reporting 'being ganked' necessarily equate to admitting a fair defeat. It could not be claimed that because a player could ambush others of lower level, that player was 'good at' PvP. Despite these negative connotations, I observed players referring to their own actions as ganking on some occasions without apparent remorse, and even when their actions would be considered legitimate. For example, when setting out to hunt for members of a guild with which we were at war, one of my guildmates declared "time to go and gank some noobs!" in our voice chat - despite the fact that we had chosen to seek out people near our own level rather than weaker opponents.

Ganking was common on my server. Guild warfare provided legitimate targets, and although being ambushed was irritating it was usually reported to warn other guild members or to request vengeance, rather than complained about as an injustice. Some players preferred to strike more randomly, and these were likely to select targets who were of a significantly lower level and unable to defend themselves effectively. One area of the game world, Lumbertown and the nearby Fey Forest, very quickly became notorious for this. It was the first low-level area where PvP was enabled (the introductory 'noob island' was a safe zone for new players), and so characters there were generally the weakest possible targets. My first death in TERA occurred there when my priest was cut down in a single blow by a higher-level slayer with a two-handed sword. Lumbertown became so notorious on my server that protecting it was a major political issue in the first vanarch elections, during a period when certain high level characters would stand on the bridge that was the main path in and out of the safe zone, and attack any player who tried to pass.

Although barring the Lumbertown bridge was clearly unfair, it was enjoyable for players who liked to prey upon those of lower levels, and accepted by many others as a part of life in the game. Some even saw being terrorised by stronger opponents as a sort of rite of passage, part of the process of levelling up when one was new to the game. "I enjoy pvp cause when you're 60 you get to do the same crap that the people did to you when you were 15 and they were 60," one player stated in a discussion of what constituted 'fair' player combat. This kind of view normalised 'unfair' attacks as part of what PvP meant. 
It should be noted at this point that death in TERA was objectively little more than an inconvenience. A character who died was given the option of being resurrected at a nearby town with low health and stamina. Stamina took some time to recover, which might keep a player from the game for a few minutes, but resurrected characters kept all of their equipment and would recover full strength. ${ }^{22}$ Being killed could be embarrassing and frustrating, but it was not a serious setback in a character's development. Killing someone's character was thus (in stark contrast to 'real' killing) a fairly mundane social action, especially in the atmosphere of a PvP server. In this sense execution could actually serve as a light social sanction, for example when a player unfairly 'ganked' another player only to be killed in vengeance some time later by the victim's guildmates. Ideally, this discouraged things that were viewed as illegitimate by making routine play more difficult for offenders, especially if their victims had allies (cf. Jakobsson and Taylor 2003:83). In practice though, players who wanted to dominate others in this way would do so despite the likelihood of retribution.

Choosing to play on a PvP server meant accepting the risk of being attacked at any time, including when it obstructed other activities. Some cited the need for constant alertness as part of the appeal of this style of play, making it more engaging where programmed encounters could otherwise become repetitive. A guildmate explained that "a lot of it is that fact of knowing it's an actual person playing that char[acter], instead of a CPU 23 whose AI's not all that challenging." The social element of competition and measuring oneself against others was valued, with status being more clearly measurable than in offline life through scores and rankings which quantified performance. Nonetheless players did not all agree on what constituted 'good' PvP. Some felt that it should be reasonable to expect PvP between relatively equal characters, and that those who stalked character of a much lower level were playing 'wrong'. Others were not so concerned with honour and insisted that unfair fights were also part of the game. "When it comes to mmo gaming keep in mind if you join a pvp server that means your not gonna rage about getting killed cause you weren't forced to play one," a player said in response to another's expression of

\footnotetext{
${ }^{22}$ Crystals (enhancements added to items) could break upon death, but in most cases these were easily replaceable.

${ }_{23}$ 'CPU' was slightly old-fashioned video game terminology for a computer-controlled opponent, as opposed to another player; 'AI' here means 'artificial intelligence', the opponent's programmed actions and responses.
} 
frustration in chat. "If it offends you don't roll pvp, seriously." Such players embraced the 'dog eat dog' nature of competition often present in capitalist societies (where any advantage gained within the rules was legitimate, ethics aside) as part of their gaming, while those concerned with fair fights wanted a more controlled, idealised or 'pure' competition of skill.

\section{Code and Conduct}

"I tell you something, if you got something out of killing people in your level bracket, level 6os would be killing level 6os in the level 6o continents, and pvp would be a lot more dynamic at lower levels."

(Area chat)

Players could contest the right and wrong of PvP play because it was not always clear what the rules were. In fact, there were two quite different kinds of rules at work: those coded into the software that constituted the game's world, and those considered rules but not enforced through code. The safe zones on PvP servers were an example of the former kind; the developers chose to make it impossible for players to attack other players in the vicinity of towns and camps. This rule could not be disobeyed, because it was built into the very fabric of the game world. In effect this was less like a government making a law and more like a world's creator defining a law of physics. Players could be creative in how they made use of these features of the game world - for example, I saw groups of players fighting their guild's enemies just outside a camp so that their healers could support them from within the boundary, where they were invulnerable. Nonetheless coded rules were features of the environment, which players were automatically and absolutely bound by when they played (chapter six will explore the relationship between those who had access to the code, and those who only acted within its structures).

The second type of rule had an authoritative form in En Masse Entertainment's terms of service. These could not be enforced in such infallible ways, usually because they were based around the most uncontrollable element of any computer system, the users. En Masse employees could ban players who were caught or reported for breaking such rules, but it was impossible to monitor the whole game all of the time. 
These rules of conduct were more like the laws made by governments in the physical world, in that players could choose to disobey them if they were willing to face the consequences of being caught. In addition to the terms of service there were individual players' (or groups of players') interpretations of the game and how it should be played. These were the most contested, and they caused difficulty because while the authority (En Masse) made clear rulings about some things, they left other matters ambiguous.

Montola (2012:305) describes games as falling into three categories with regard to their rules: referee-centric, player-centric, and designer-centric. TERA was designercentric, in that En Masse's official decisions (enshrined in code) overruled player interpretations of the game. The designers had the final word on 'proper' play, and through regular updates their stance could change and react to what players were doing. Despite this, there were areas in which developers could have modified the game to make their ruling on an issue clear, but they chose not to. Having worked with online game developers, Zabban reports that "player calls for referees have to be carefully considered by designers, who precisely do not want to arbitrate heterogeneity in their subscribers' game practices" (2011:8). For example, En Masse could have made it impossible for players to kill other players if there was a difference of more than five levels between their characters, enforcing a 'proper' form of PvP - but they did not. By not explicitly prohibiting something, they seemed to implicitly sanction it, or as Zabban's developers succinctly put it, "if the system is allowing it, then you are allowed to do it" (2011:8).

Complaints (and dismissals of complaints) about PvP conduct illustrate well how there could be multiple interpretations of how the game was supposed to be played, what goals were legitimate, and what constituted a wrong against the community (issues on which Elleon and Jelena's conflicts often hinged, as described in chapter four). On matters where En Masse remained silent and enabled multiple styles of play within the game, conflicts could break out when one player's transgression was another's fair play. As a game, it seemed that the rules should be clear, but as a virtual world more options enriched player interaction and allowed players greater agency. It is possible that a greater consensus would have developed in TERA's community over time, but in its first months players brought together expectations 
based on their previous experience in a variety of other games with different local cultures of their own. Separating servers into designated PvP, PvE and RP versions of the game helped to alleviate these issues by grouping similarly motivated players, but I suspect PvP was the most contested of these three broad styles of play. 24

As a final note, the ideal of meritocracy discussed above in relation to game balance was also prominent in discussions of PvP legitimacy. It was widely held that fighting against players required a much greater degree of skill than fighting programmed monsters and other enemies. Losing to another player (ideally) brought "a sense of determination" or a desire for "payback", motivating one to improve one's skills and win next time. The taunts and insults common between warring players reflected an atmosphere of competition which the comparatively static programmed challenges could not inspire. Because of this ideal, complaints about illegitimate PvP were often met with comments like "you only say that cause you're a bad", that is, an unskilled player. As PvP was thought to require skill, and skill was the worthiest quality in a player, doing badly was assumed to mean that a player was not skilled enough and thus should perhaps not be participating in this form of play in the first place. "Roll $\mathrm{PvE}$ and you'll never get killed" was the final suggestion given by one player to another who complained of constant attacks. The ideal of the skilled prevailing could thus be used to justify what some saw as unfair or unsportsmanlike practices in PvP, on the grounds that those who were unable to keep up were themselves at fault for choosing to play on a server where the combat was beyond their abilities. As mentioned above, however, few if any TERA players would admit that their own skills had proved insufficient, even when they were beaten.

\section{Conclusion}

TERA players had an ideal vision of how online games should be fair, in which success was based on the merit of the player - their skill - and not on any feature of the software or game design, which was expected to be neutral. It was En Masse's responsibility to maintain balance and ensure that all players had equal

\footnotetext{
${ }^{24}$ I did not play extensively on a PvE server or at all on an RP (role-play) server, so my experience is limited to PvP environments. Because PvP involves other players to a greater extent, however, it provides more opportunities for misunderstandings, different interpretations, and the sense of having been wronged. In other matters, criticism was more likely to be directed at En Masse for their design choices, rather than at other players.
} 
opportunities to succeed regardless of the race and class choices that they made when initially creating their character. Players wanted control; they did not approve of too many random elements in the game, or of anything perceived as systematic advantages or disadvantages. Agency, as the capacity to act as one wished and to make a difference, was important to their enjoyment; they wanted to pursue 'projects' within the game (cf. Ortner 2006) and achieve through their own ability. This goal was consistent with a capitalist consumer drive to be better and have more, but through the software more egalitarian starting conditions could be enforced than those found in the physical world. Players paid money to assure access to a controlled, idealised and 'fair' version of the great game of consumer competition, one where hard work was always fairly rewarded and outcomes were predictable. They could demand that En Masse 'fix' what they perceived as design problems because they paid a subscription fee, meaning they considered themselves entitled to exactly the same opportunities as other paying customers. The complexities of the customer-developer relationship will be explored further in the following chapter. 


\section{Developers and Players}

Alongside the categories of class and play style described in the previous chapter, there was another pertinent division in the world of TERA: that between the company which created the game, and its players. Neither group could participate in the game world without the others - a virtual world without players is just "an empty data warehouse" (Bell 2008:2). Yet there were inevitable tensions between developers and players of online games, tensions which En Masse Entertainment went to some effort to mask in their relations with the players of TERA. Having extensive control over not only the 'rules' but the physical possibilities within the game gave its creators a degree of power and control that cannot be rivalled in offline societies. Players proved to be far from powerless, however, and were well aware that their position as customers gave them significant leverage over En Masse, who operated the game as a business and needed to make a profit from players' willing participation. In this chapter I will discuss the ways in which developers' and players' interactions created a specific kind of commercial relationship, one in which consumer agency was an important mode of action.

\section{Power Relations}

"I honestly doubt Tera is going to see any more of my money. The staff plain and simple doesn't listen to us and our complaints that we've been making for months." (TERA forum post)

There is no perfect analogy for the relationship between an online game company and a game's players. The creators and managers of an online game have a degree of control over their constructed worlds that is quite unprecedented: they can define the virtual equivalent of natural laws. Under even the most totalitarian government citizens can choose to disobey authority - even if disobedience is likely to lead to their death, they have the ability to choose and the potential to act otherwise. In other words, they can exercise agency as human beings. In an online game, if designers decide (to take an example from TERA) that players cannot attack other players within a certain distance of a camp, it is physically impossible for players to act against this ruling. There is no choice to obey or disobey. Such design decisions 
can be enforced through code, written into the software which defines everything in a virtual world, from the environment to a player's own avatar. The only way that players could counteract a programmed rule is by hacking the software and gaining access to the company's servers, which would require exceptional technical skill and the bypassing of significant security. Moreover, doing so would violate the terms of service of the game and the offender would lose the right to access the game world at all, nullifying any gains they made.

'Nerfing'25 provides a vivid illustration of the power designers could exert over their game worlds and the players who participated in them. Players were often upset when updates changed the parameters of the game without warning, disrupting the strategies that they had built their characters around. What worked one day could be ineffective the next. Changes were implemented through game updates as developers saw fit, effecting sudden, deliberate alterations which were imposed on the world from outside its boundaries. Because playing TERA required connecting to En Masse's servers, there was no option to ignore or refuse an update: everyone would play under the same rules, but no player had access to the software code where those rules were defined. If players wanted to participate in TERA at all, they had to accept any changes that the developers might choose to make to their world.

There were two important factors which balanced the lack of power that players had over the game itself. Firstly, the game was not essential to any player's survival or well-being. Unlike the kinds of land-bound peasants whose resistance strategies James Scott has written about (1985), players had the ability to leave a game world at any time. One of the advantages of social activity on the internet is that it can be entirely detached from one's 'real world' life, allowing greater freedom for exploration, identity play, and provocative modes of behaviour such as 'trolling' (cf. Donath 1999:43) without consequences in other spheres of life. In most cases participation or non-participation in a game would not have major consequences for one's offline life and relationships, and although emotional investment can be significant, no player is dependent on a game world for food or shelter. As the oftquoted (though overly dismissive) adage has it, "it’s just a game.” Secondly and very

\footnotetext{
${ }^{25}$ As defined in chapter five, to 'nerf' something meant that an official change was made to the game which rendered some aspect weaker or less effective than it had previously been.
} 
much related, players entered an online game as customers. They chose to play the game and paid for their access, usually in the form of a subscription fee. Game companies were businesses, and needed players to continue to spend money on their games if they were to profit from them. The combination of these two advantages meant that TERA players always had an ultimatum at their disposal: satisfy us, or we leave. Every dissatisfied customer could reduce a company's profits and so, for all the control that they had over the conditions of their game worlds, companies like En Masse did have to take their customers' opinions and desires into account (cf. Blanke 2007:185).

Players were very aware of their positions as customers (rather than just 'players'), and their expectations of the game reflected a sense of entitlement associated with a customer ethos. As Annemarie Mol observes, "as a customer you are made active rather than passive. It is up to you" (2008:16). Players became the choosing agents, with game companies competing to offer the best product for their selection. Based on their own broad knowledge of MMORPGs they critiqued TERA, or critiqued other games in light of TERA - for example the common accusation that WoW was a 'carebear' game while TERA was less forgiving, and therefore suited to more advanced players. They were also quick to point out flaws and to demand that En Masse 'fix' them, as previous chapters have shown. It was not up to the players to make TERA what they wanted it to be, because customers are not expected to work for their product - they pay their money, and expect it handed to them in return (cf. Delucchi and Korgen 2002). This mode of thought assumes that the companies need their customers (not the other way around), thus empowering the consumer. I will argue that this led TERA players to consumer agency as an effective mode in which to interact with developers.

This view of the consumer as empowered rather than helplessly controlled is a development in consumer culture. It may seem to come easily in an area such as gaming, where the product chiefly provides entertainment and is thus non-essential, but scholars have noted similar attitudes emerging among consumers more generally. Applbaum (1998:323) sees a shift in Western thought, from consumption as a necessary evil to consumption as a way to strive for the best in everything. Jacobs observes that in contrast to earlier scholars who decried the hedonistic 
indulgence of consumerism, in recent times "historians came to see consumers as actively struggling and contesting their role in society" (2011:565) and "in the twentieth century, this consumer impulse has generated a rich and varied politics" (567). TERA's players were certainly not passively receptive consumers, but rather very active agents who wanted to influence the services they paid for. 'Real world' consumer activist movements (e.g. Glickman 2009, Kozinets and Handelman 2004) are other instances where people have chosen to embrace the role of consumer and use it to influence the world. Nor were TERA's players unique among gamers: MMORPG blog 'Massively' hosted an article in 2013 advocating that players use their money to force developers to show them greater respect (Royce 2013).

The MMORPG market was highly competitive, with a large number of different games available; at least four major release ${ }^{26}$ were heavily discussed in TERA during my fieldwork period of only four months. If players felt that the service they were receiving in their current game of choice was sub-optimal for any reason (cost, customer service, game balance or frequency of updates, to name just a few examples), they always had a number of other, similar games to choose from. I often encountered groups of friends who had shifted games together, including Elleon and Jelena, and Sikander and his friends (see chapter four). TERA was also host to branches of a number of trans-game guilds, who maintained their own community websites unconnected to any particular game. Having their own websites and forums allowed players to organise independently of the tools that individual game companies provided, and in-game friends were no longer tied to a particular world. This enabled players to be highly mobile between virtual sites, as described in chapter three, and this meant that threats to leave a game were not empty threats.

\footnotetext{
${ }^{26}$ Star Wars: The Old Republic had been a high profile release some months before, Guild Wars 2 and The Secret World were upcoming, and Diablo 3, although not an MMORPG, was played online and was much discussed in TERA chat channels.
} 


\section{The Other Meta-Game}

"It makes me mad when people add me to friends list, view my location, delete me from friend list immediately, arrive at my location, then kill me. This is an exploit, and it needs to be fixed."

(TERA forum post)

In gaming (both online and otherwise), the term 'meta-game' refers to the everchanging development of shared strategies and counter-strategies by a knowledgeable player community. Meta-gaming affects how the game is played but takes place outside of and around it. In TERA for example, critical hits were a popular focus of player strategies by the end of my fieldwork, and players debated how best to maximise and use them at length in a number of contexts. I interpret meta-game as 'the game of playing the game', in which a player uses information from outside the game itself (such as an understanding of the current favourite strategies among players) to devise the most optimal approach at any given time. Here, I want to suggest that there was also a second kind of meta-game: trying to outsmart the developers and their designs. This form of meta-gaming shows the playful approach that players took to their position in TERA: they tested the integrity of the system that developers had built by pushing back against the rules, trying to find a trick that had not been anticipated. The following story, which I heard after my main fieldwork period ended, illustrates how this could happen in TERA.

One feature which had been advertised for TERA but which was not available at the game's launch was the ability to win vanarch positions through PvP combat, rather than through voting. As originally conceived these combats required the 'battlegrounds', which would not be available until August. As a temporary measure until then, En Masse implemented a special kind of guild warfare on June 29th. Guilds could select either voting or guild war as their method of competition, and of those who chose war, the guilds with the highest resulting scores could win a warfare-based province. One guild ${ }^{27}$ immediately saw a loophole: rankings were determined by the number of enemy kills a guild had accumulated. This guild split its membership in two, forming a temporary second guild which proceeded to 'feed'

\footnotetext{
${ }^{27}$ I was told of a specific guild, but the forums suggest that this occurred in a number of cases across servers.
} 
them kills - allowing themselves to be killed repeatedly, with the original guild earning points each time. Using this tactic, the guild could engineer an extremely high score for itself without ever having to face its real opponents.

This is an example of an 'exploit', an action which takes advantage of something that the software allows (thus technically not breaking any rules) but obviously goes against the intentions of the system. Guild versus guild warfare was intended here to award a vanarch position to the guilds who performed best in PvP. The tactic of splitting one's guild and setting up a straw man to fight bypassed this, and entirely circumvented the ideal of meritocracy described in chapter five. That players took to such behaviour so easily reflects the playful disposition which they brought to the game. As Victor Turner put it, "there is no sanctity in play; it is irreverent and is protected in the world of power struggles by its apparent irrelevance and clown's garb” (1985:265). In TERA, players were not concerned about the consequences of an exploit: if the game's software let them do it, they could not be doing anything wrong. If they upset the system, it was En Masse's responsibility to fix it. They would take whatever advantage they could before the opportunity was eliminated, and having technically acted within the rules, they could usually not be punished.

Michel de Certeau calls this kind of opportunistic behaviour 'tactics', a characteristic mode of action for people who live within the influence of more powerful institutions; "the space of the tactic is the space of the other" (de Certeau 1984:37), as when an employee surreptitiously conducts personal business while at work. Tactics operate in the moment, without thought of long-term consequences or sustainability; they are reactive, seizing small advantages where they can, just as gamers' exploits do. The institutions which dominate the context of such actions are too cumbersome to act spontaneously, but they are able to employ more methodically organised strategies which require:

"a place that can be delimited as its own and serve as the base from which relations with an exteriority composed of targets or threats (customers or competitors, enemies, the country surrounding the city, objectives and objects of research, etc.) can be managed" (de Certeau 1984:36, emphasis in original). 
De Certeau discusses the influence that 'cultural producers' exert through mass media; in TERA, it is clear that the game world was En Masse's 'place', their home ground where they had control and from which they could interact in controlled ways with customers. If players wanted to participate in the world of TERA, they had to do so on En Masse's terms. However, de Certeau also emphasises that 'cultural consumers' are not entirely passive. In meta-gaming, we can see what TERA players 'made of' the game they were presented with (cf. de Certeau 1984:31), as consumer agents.

Turner also wrote that "playfulness is a volatile, sometimes dangerously explosive essence, which cultural institutions seek to bottle or contain" (1985:263). TERA was in itself just such a way to 'bottle' playfulness - channelling it into approved activities from which En Masse could profit. This is an example of Malaby's (2009a) insight that playfulness can be harnessed by companies and institutions in a similar way to ritual, another potent mode of human experience. Although selling play made TERA an appealing product (or service), it also made En Masse's customers dangerous to them through the "volatile" nature of play. In encouraging improvisation and creativity, play discourages simple obedience to rules. Part of what made TERA enjoyable was learning to manipulate the game world and its systems, but this also trained players to look behind the curtain and think about how the game actually worked. The omnipotence of game designers within their worlds was a double-edged sword: because they could control and limit almost everything in the game, anywhere they failed to explicitly and firmly constrain, players assumed they were free to act. Hence what I call 'the other meta-game': finding technically legitimate ways to manipulate the game's design in ways that were never intended - or anticipated - by the designers.

Developers' extensive control actually made it easier for players to claim innocence in cases like that above. In taking advantage of this (presumably) unforeseen loophole they had not hacked into En Masse's servers, or broken any terms of service or rules. How, then, were they to know that this wasn't intended to be a valid strategy? They could always fall back on the position that 'if they didn't want us to do this, they could have stopped us.' As I argued in chapter five, players considered it En Masse's responsibility to ensure that their game was played the way it was meant to be. En 
Masse, a group external to the player base, were in charge of maintaining the structures of the game (environment, classes, combat mechanics); within these structures, if players found an opportunity for advantage, they would seize it. For some people, it was all part of the game.

\section{Play vs. Business}

"For a first time publisher, EME has done pretty well, but they place far too much emphasis on throttling the content to try to keep people around. It's blatant, feels cheap, and is infuriating."

(TERA forum post)

Thomas Malaby (2009a) has emphasised play as a 'mode of experience', a playful 'disposition' which can be applied to any activity, not just those that are intended as games. Knowing that they were ('just') games, players typically approached MMORPGs in this playful mode. Malaby describes the 'play element' as "marked by an interest in uncertainty and the challenge to perform that arises in competition, by the legitimacy of improvisation and innovation that the premise of indeterminate circumstances encourages" (2009a:210). This sense of challenge and creativity was very much evident in TERA, particularly in PvP, but it was not just players' relations with other players which were playful. By virtue of it being a game (and perhaps doubly so for being online), everything in TERA was ultimately trivial - which is why players could leave the game without major consequences in the rest of their lives. Even as relationships and prestige were treated seriously within the bounds of the game, "purposes, consequences, statuses, skills and so forth are to be understood, for the most part, only within the context of the event" (Henricks 2008:177), or in this case the game world. It allowed experimentation, with the fun being in seeing how a strategy worked out (or, as above, how much one could get away with) without having to worry about wider implications in one's life.

Here there is a disconnection between players and game creators, not just in terms of power but in terms of their attitudes - the modes in which they approached the game. For designers, managers and developers, TERA was business. Where players could act recklessly because there were little or no 'real world' consequences, those 
who worked at En Masse Entertainment had their pay, their jobs and their reputations staked on the success of the game. The "indeterminate circumstances" were full of risk for them; En Masse needed to make a profit. Control and consistency were preferable. The differences between the modes of 'work' and 'play' inevitably divided those who built, managed, re-balanced and updated the game from those who played it, manipulated it, and exploited it.

MMORPGs were 'organised' play of the kind that "are frequently controlled by nonplaying administrators and follow procedures that maximise the benefits for the sponsors they represent" (Henricks 2008:169), the key being that the organisers were a separate group to the players (Henricks cites casinos as an example of this). While the player might enjoy the experiences of reaching goals, overcoming obstacles or successfully co-ordinating a team, the developer had to be preoccupied with making sure that the balance or 'flow' (Csikszentmihalyi 1990) of gameplay provided for players was just right in order to keep them entertained. Miller suggests that part of the appeal of play is that "there is somehow something very desirable about acting, at least for a time, in a framework designed by ourselves rather than by the existential forces that run most of our life" (1973:97), but the rules and conditions in TERA were not devised by the people who had to play by them. They came from outside, from the kind of corporation that arguably is an existential force for many of us. Like casinos, developers were to some degree an opponent to be beaten at their own game.

En Masse Entertainment went to some lengths to mask the tension caused by this disconnection between business and play. On their website they repeatedly emphasised that "we're gamers", "the game comes first," and "games are who we are." They used casual language, gamer jargon, and humour to portray themselves as on the same level as players. Patch notes, which listed the changes (great and small) made in each update, often reflected this predilection for humour:

"Fixed a bug where guild-versus-guild battles could not be declared on guilds with names longer than 32 characters. Your awkward battle cries will save you no longer." (Patch 17.25.02) 
"The healing NPC28 will no longer steal the boss kill from players in Karascha's Lair. Why do priests always want to DPS?”29 (Patch 17.29.01)

Known Issues: "When trying to look at the game stat UI30 tooltip, the tooltip occasionally appears in an abnormal location when the pointer is near the UI boundary. The tooltip just appears in an abnormal location on your computer screen, though, not "inside your refrigerator" or "on a billboard in Lima, Peru." Those would be really abnormal locations for a game stat UI tooltip." (Patch 17.32.03)

"Fixed the problem of warrior tanks occasionally not receiving Instance Matching buffs. Contrary to rumor, lancers were not behind it." (Patch 17.32.04)

As these examples show, En Masse employees often made reference to in-game events and tropes, showing that they were in touch with how their game was actually being played. They downplayed the division between players and developers, instead portraying themselves as approaching the game with the same playful attitude that their customers adopted. They were all in it together, and all wanted TERA to be the best game it could be. However, players' awareness of their roles as customers meant that they often pushed back against this pretence, accusing En Masse of making decisions based on a desire for profit (rather than on making the game enjoyable) when they were dissatisfied. The differences between the two sides were never actually forgotten, and in fact many players seemed to take an active interest in making sure that the distinction was clear. Forum posts such as the one at the beginning of this section, from those critical fans who could be labelled as 'voicy consumers' in marketing terms (Foster 2007:714), clearly showed their awareness of how players could be manipulated or exploited. This was part of a broad knowledge about online games in general, born of extensive experience.

\footnotetext{
${ }^{28}$ Abbreviation for 'non-player character'.

${ }^{29}$ Abbreviation for 'damage-per-second', meaning to focus on dealing damage.

${ }^{30}$ Abbreviation for 'user interface'.
} 


\section{Virtual Structures}

"The sign enjoys an actual being, in praesentia, only as it is inscribed in human action. As a scheme of relationships between symbolic categories, the "system" is merely virtual."

(Sahlins 1982:43)

When we refer to 'social structure' or 'cultural structures' in offline, 'real world' societies, we refer to something which is abstract and imagined. Although such structures do shape and affect people's actions, they are not located externally to a group of people who live by them; they do not have an objective existence 'out there'31. Rather, as theorists such as Giddens and Sahlins have argued, social and cultural structures exist in the world only as people put them into action. Through many instances of practice applied to specific circumstances, people effect change in the long run. Sahlins' (1981) discussion of Hawaiians' contact with Europeans and subsequent transformation of their own cultural system is an excellent illustration of this concept. The Hawaiians applied existing concepts, analogies and relationships in ways consistent with tradition, and yet the strategic re-application of these traditions to new contexts brought about new ways of doing things. Through this, the culture was able to adapt to new circumstances.

In the offline societies with which we are more familiar, then, structures emerge out of a group. They have been shaped by the needs and practices of our predecessors and are being shaped by the current group members as they apply the structure(s) to their everyday lives. MMORPGs are different in a number of key ways. First of all they are constructed environments, bound to some extents by the limits of hardware but not by the biological factors that all offline societies have to manage: birth, food, reproduction, death. Certain physical assumptions, like requiring bodily proximity for co-presence, become invalid through software. Games are designed - by people outside of the player community - to work in certain ways and foster certain forms of social life (such as guilds, or server community) through incentives or manufactured needs. Each game world has a beginning, a point at which developers decided to launch the game and open it up to participants, and each character has a known

\footnotetext{
${ }^{31}$ The classic analogy is Saussure's distinction between language (and abstract system) and speech (specific acts); see the introduction.
} 
moment of creation and definition in which its controller has significant input. The design and launch are not organic processes developing out of practice, but rather plans and calculated decisions - strategies, in de Certeau's terminology. The nature of change is different too: updates come as discrete, scheduled sets of changes, redefining the world each time the software is modified. Again, things do not change by process in the continual sense, they change in clear, distinct, deliberate steps.

I do not mean to deny that MMORPGs have cultures or social structures that emerge organically among their players - any number of studies have described just such things. Sewell argues that what enables people to act effectively in a context is knowledge of its rules, and "the usual social scientific term for "what people know" is "culture"” (1992:7). What I do want to highlight is that these player-based structures and even trans-game norms are not the only sources of 'structuring principles', as Giddens terms them. The software itself - the code - is a prior structuring element, which shapes what players do and what they are able to do in a way which is difficult to compare to society offline. Everything in TERA, from the scenery to forms of embodiment, the availability of wealth to the capacity to kill someone, is determined by deliberate design. If agency, in turn, is "the socioculturally mediated capacity to act” (Ahearn 2001:112), here it was also a technically mediated capacity, limited firmly by what the software allowed. This made the possibilities open to players much more finite from the start.

This does not mean that practice-based theories of social structure have no place in online games; players can still effect change through their practices, especially in their roles as customers. Code was not the only thing which determined how the culture of a game developed over time. As Giddens wrote, "human history is created by intentional activities but it is not an intended project; it persistently eludes efforts to bring it under conscious direction” (1984:27) - or, as Morningstar and Farmer joked of their early experiences running virtual worlds, "in the most carefully constructed experiment under the most carefully controlled conditions, the organism will do whatever it damn well pleases" (1991:288). Despite the restrictions under which they operated, players were still agents, able to choose and act - and their choices and actions did not always match up to what developers predicted or encouraged. From a business standpoint, is a marketer's job to predict the practices 
of consumers and react to them accordingly in providing their products (Blanke 2007:197). Just as practice feeds back into structure and each changes the other, customer opinion and marketing rhetoric do the same. In the world of TERA, the processes seemed to be one and the same.

\section{Choices and Demands}

"As of right now TERA is moving in the direction of failure ... Here is a list of things En Masse has to focus on these fixes/changes if they want to save their game." (TERA forum post)

In theory, players could effect change in an MMORPG in the same way that they could offline, such as in Sahlins' Hawaiian case. Players could simply choose to act differently, to do some things (and not do others) in defiance of existing structural 'rules' if it better served their own interests. Although some behaviour in TERA was incentivised by design, it did not necessarily preclude the alternatives. If TERA's instance matching tool caused problems for players, for example, they could decline to use it and instead turn to chat channels to seek group members, or form groups within guilds. In turn, if En Masse noticed that no one used the instance matcher, they might reconsider how it worked and re-design the feature to fit more closely with player practice. It was in the company's interest to adapt the game world to what players seemed to want or need as time went by, even if what players said they wanted could be rather inconsistent; in marketing, "one must give customers what they ask for, not what works best or costs least” (Applbaum 1998:329). In the later stages of TERA's 'life' (notably around October after the servers were merged) En Masse were indeed very responsive to player feedback.

Much of the time, however, I did not see players taking the initiative to change their practices in this way. They were very responsive to the way the game was designed and tended to use any routes which were incentivised. The continued use of the instance matcher despite the problems described in chapters three and five illustrates this clearly - the instance matcher was convenient, whereas guild groups, although considered superior, required more organisational effort. Certain kinds of action, although optional, were subtly or overtly encouraged - and 'fun' was not 
always an overriding factor. The possibility of gaining unique rewards, rare items or gold were incentives, as was anything which allowed a player to attain these prizes more quickly or cheaply. Being very knowledgeable about their game, TERA players quickly discovered the most efficient ways to get what they wanted (hence my guildmates' dismay at how slowly I levelled up). Few would sacrifice their own shortterm progress, or that of their guild, in order to change the system in the long-term, especially when they were in competition with other players and did not want to fall behind the community standard. The focus on the here and now is perhaps not surprising, given how many players did not play for more than a few months before abandoning the game.

Sherry Ortner (2006) discusses agency in terms of 'projects', and an individual's ability to conceive of and pursue any projects that they may wish to. She rightly highlights that those with power in a society can generally pursue any project they choose to - in TERA terms, the designers can make any changes they wish to make, with sufficient time and resources. Those who have less power are more limited in what they are actually able to do. TERA placed very real limits on what projects players could choose to pursue within the game world, because the software medium itself defined rules within which they had to operate. Additionally, the game provided players with certain sanctioned projects which they were encouraged to pursue, just as culture does in Ortner's discussion. She gives the example of a man who wants to increase his status among other men; this project is encouraged and reinforced in his culture, making it a desire people can understand and share. Comparably, the project of reaching the maximum character level in TERA was so ubiquitous as to be assumed of all players, and the game was clearly designed to encourage and enable it. Other projects were accommodated in the design but not taken up by most players (such as vanarchy), and still others were pursued by players with relatively little support from the game's structures (such as running a socially successful guild).

If "agency refers not to the intentions people have in doing things but to their capability of doing those things in the first place" (Giddens 1984:9), then players certainly had their agency limited within the bounds of the game - En Masse had an unusually powerful ability to curb agency through their control over the game world. Certain projects that players might like to pursue in their style of play (for example, 
ambushing one's guild's enemies while they were within a town) were denied to them by the way the world was built. Nonetheless, as we would expect, people found ways in which they could pursue at least some of their own projects within this system. Elleon and Jelena's attempt at joint leadership was one such project, although it failed under the combined pressures of the game's mechanics and social tensions. Their example well illustrates Ortner's reminder that 'games' of power and agency occur not only between large factions like players and developers, but also on the micro level between individuals within a group (2006:151). Exploits were also ways in which players worked within the game, but around or against it at the same time.

That said, TERA players were often not at all passive or indirect about trying to change the game. The official forums hosted by En Masse were frequently used by players to voice their complaints, suggest alternative designs, and issue ultimatums. Demands and imperatives were common, with players clearly showing that they felt entitled to dictate to En Masse how the game should work. A sampling of recent forum thread titles from one day included:

Fix your servers EME

Remove Desperate Resolve buff yesterday

Nerf archers

Don't nerf archers $3^{2}$

Here we return to the fact that TERA players were empowered chiefly through their position as paying customers. They were bold and forthright with their feedback and often fell back on the threat of leaving should their complaints not be addressed. The ease and frequency of players' movements between games (discussed in chapter three) meant that they were well-informed about their options and the state of the market. TERA was host to a particular form of consumer agency, in which players acted through their position as customers. Even though many individual developers showed care and concern for the game itself, En Masse as a company ran it in order to make a profit. The greatest influence that players had was as the source of that profit, and so they used that to pressure the more powerful company into conforming to their wishes. Developers had the greatest control over player actions within TERA

\footnotetext{
${ }^{32}$ Forum thread titles from http://tera-forums.enmasse.com/forums/general-discussion (accessed 10/09/12).
} 
itself, and constraints were accepted there as a legitimate part of the game. Consumer agency operated outside the bound of the game, in forum discussion, meta-gaming, and most of all in the purchasing choices that players made. Although it did not allow players to change the software, it gave them leverage.

\section{Conclusion}

Relations between TERA's developers and players were mutually beneficial, but full of tensions. Players were customers of En Masse Entertainment, and knew that the company needed the fees they paid to make a profit from running the game - which was, after all, its ultimate purpose. As customers, they felt entitled to make demands and issue ultimatums in order to coerce (if necessary) En Masse into making the game into what they wanted it to be. Aware that game companies had different interests, players were watchful for design elements which seemed primarily intended to ensure that they continued subscribing, such as 'speed bumps' which artificially slowed progress toward a goal. The distinction between 'customer' and 'service provider' was always present. Within the game player agency was limited to an exceptional degree by the software, which only allowed actions which developers had chosen to build into the game; 'structure' was much more real and constraining than it is in physical world societies, and because it was deliberately constructed it chiefly served the company's interests. Players could act outside of the software, however; by using the commercial nature of the game to their advantage, players who were not able to change the game themselves could quite effectively demand that it be changed for them. They perceived their power as consumers, and used it to compensate for their lack of control within the game. 


\section{Conclusions}

Structure and agency are interlinked forces which exist alongside one another: structures inform and shape the actions people take, while the actions of individual agents give structures existence in the physical world. In the preceding chapters I have explored how these forces and their interplay were present in TERA, a world designed and built by one group of people for another. Who is involved in asserting agency, and who in building structures, in such a setting? Considering these questions can help us extend the concepts of structure and agency to better account for how technology is opening up new ways of living, which may not abide by all of the assumptions upon which our concepts are built.

TERA was one of a large number of massively multi-player online games released during 2012, and one of the many more in its genre released in the last decade. As a role-playing game in a fantasy setting, it was typical of its contemporaries; as an action-based game that required active aiming and dodging, it stood out as different. Introduced into a very competitive market, it went through a life cycle of growth, decline, and a struggle for stability in the midst of constant change which seemed to be common for new games. Each player that entered the world of TERA did so with the payment of a monthly fee, which placed them in the position of customers to En Masse Entertainment, who provided the game and its upkeep as a service. It may have been deemed ready for release, but TERA was never a finished product - it developed and evolved in response to the actions and responses of the players who inhabited it.

To participate in games in the physical world players consent to a specific set of rules, and any changes to these rules can be decided by a consensus among players (for example the unofficial 'free parking' rule in Monopoly). TERA could not work this way for two reasons: firstly, consensus could not be gained from the thousands of people on a server; secondly, the players did not control the rules of the game - En Masse Entertainment did. This game was 'organised play', designed and presented to its players from one party to another, but players were not simply passive receivers of a product. They were active participants in its development. Engaging with their gaming via capitalism, players acted as empowered consumers, using their position 
to make demands of the ostensibly more powerful company. They emphasised their ability to move and choose freely from among competing games, often displaying evidence that they were easily bored and focused on the short-term. In contrast to other virtual worlds which placed an emphasis on creation, TERA players seemed largely disinterested in working to get more out of the game for themselves; they were paying for a service and expected the company to package entertainment for them. This produced what gamer blogs referred to as 'theme park MMOs', full of programmed, ready-to-play attractions.

Locked out of the code which defined the game world - from its environment to the terms of gameplay - players used their commercial relationship with En Masse Entertainment to gain some control over the development of the game. As much as they tried to de-emphasise it when communicating with players, the purpose of the game (for the company that maintained it) was to make a profit. Many players resented the idea that their play was just a means to that commercial end for the company, but it worked both ways. Players knew that the game was designed in part to manipulate them into spending money, but they also knew that they could use En Masse's dependence on their spending to manipulate the company in turn. Although the real, external, uncompromising structure of the software limited player agency in the sense of "not...the intentions people have in doing things but...their capability of doing those things in the first place"(Giddens 1984:9) within the game world, players embraced a form of consumer agency to still affect that world. Players and developers thus operated in the kind of duality (as opposed to dualism) which Giddens describes for structure and agency: not eternally opposed, but rather feeding back, each shaping the other's manifestation for the future. This relationship was conceived in commercial terms.

\section{A Capitalist Mindset}

The way that TERA players approached their online gaming aligns with a capitalist culture and a consumer ethos. Players had more power and influence over the game as customers than they did as players or inhabitants of the world, and so this was the mode in which they tended to interact with En Masse. Particularly because the market for MMORPGs has become so competitive in recent years, players were well 
aware that new companies like En Masse needed them. Beyond that, the values that players displayed in the game reflected values present in North American capitalist societies, but in an idealised form (notably in the ideal of meritocracy). All players were to be assured an equal start, but inequalities were part of the game and it was accepted that skilled and committed players would rise to the top. Achievement was reflected in possessions (enchanted weapons that glowed, rare armour) as well as in official rankings for all to see. Players would need to co-operate to work their way up, but competition between groups was an integral part of social life in the game. Many of the complications of capitalism as it exists in 'real life' were smoothed out by the control of developers in this constructed world, who could make the system more fair and enjoyable than its offline equivalents.

In addition to this, players tended to treat the fields in which they interacted as if they were markets - both when moving between games, and within TERA. Most obviously, choosing which of the many similar MMORPGs one would play was a matter of finding the product which best fit one's 'needs' (preferences in terms of gameplay). TERA players tended to prioritise an engaging combat system and/or high-quality graphics, which led them to TERA as the best choice for them. If the game disappointed them, they could rescind their subscription fees and return to the marketplace to find a game they liked better. A more subtle market mentality was evident among players in their interactions with each other: choosing a guild, or even a server, was a matter of evaluating the options and finding one that provided the best value and opportunities for what one wanted to do. These phenomena are linked by an abundance of choices, all more or less equally available through the mechanisms of the internet, and all entirely voluntary. Both to companies and to each other, players were fickle, passionate in the short-term (as chapters three and four show of TERA) but often losing interest and seeking new stimuli after a short time.

\section{Technology Shaping Our World}

Part of what makes TERA an interesting and challenging research site for anthropology is the way that it prompts us to re-consider some of the assumptions we work under in the physical world, particularly regarding structure and agency. 
Virtual, software-based environments allowed companies like En Masse to control (within the boundaries of their game) a structure much more constraining than would otherwise be possible. Whether incorporated into the game's design or brought into it by players, everything in TERA had a human origin and was shaped by human intentions; it was artificial. Virtual worlds are a particularly striking example, but parallels can be found with technological advances that are allowing humanity to determine the terms of our lives in the 'real', physical world in anew and fundamental ways. Studying TERA - an example where both creators and users were knowledgeable and comfortable with the technology at hand - can help us to explore human relations in other cases where the division between nature and culture becomes blurred or problematic.

Paul Rabinow coined the term 'biosociality' for one example of this kind of blurring, when scientific knowledge about biology and genetics informs our self-identities. He predicts that "nature will be known and remade through technique and will finally become artificial, just as culture becomes natural” (Rabinow 1999:411). Currently, medical technologies in areas such as genetics and reproduction are allowing human beings to understand and control the course of nature to unprecedented degrees. Correspondingly, in 2000 Paul Crutzen and Eugene F. Stoermer suggested that the Earth had entered a new geological epoch: the Anthropocene, characterised by humanity's lasting impact on the planet. We increasingly modify, shape and re-direct 'nature' to better suit our needs and desires rather than adapting ourselves to it. TERA may seem like a comparatively trivial example, but the game's community shows us a glimpse of the kind of society that might emerge as we come to appreciate just how much of our world can be controlled and tailored through technology. Its players were well-informed and felt empowered to take part in TERA's continuing creation, even though they lacked the access or technical expertise to modify the game world themselves.

The relationship of TERA's players with the game's developers is particularly significant in light of the fact that many of the methods we have to 'customise' nature in the physical world are offered as commercial services, as TERA was. The companies who develop and offer these technologies have the power, in constructing them, to structure their use, just as the software of TERA structured and limited what 
players could do with the game. As discussed above, TERA players found the most effective form of agency open to them was a distinctively consumer agency centred around their potential buying power. They were supported in this by the large number of competing games available and the freedom that players had to shift easily between them. Consumers do not always enjoy this degree of agency. In other industries, consumers may not have as much freedom to choose while still receiving the service they want (or need), meaning that companies may not feel as obliged to listen to or meet customer demands. How empowering consumer agency is thus depends in part on how free customers feel they are to choose if and how they enter into a relationship with service providers; where customers perceive little choice in the matter, they can feel quite powerless (Lee 2010).

\section{Where Now For Structure and Agency?}

The concept of structure and agency as a dynamic duality - two forces at work in a society which influence and change each other in a constant process - is still valid in this emerging world. What TERA and games like it challenge are the assumptions we make, when talking about these concepts, about the separation of nature and culture. In some ways, structure was more real in TERA than it could ever be in the physical world: the software placed absolute restrictions on what was possible within its bounds. Rather than emerging organically from the interactions and necessities of a society, it was designed and imposed on players by developers. At the same time, this structure was easier for players to grapple with. It was not invisible and taken for granted, but rather obviously designed by people who were still present and involved. In this context, it was easy for players to imagine that the structure could be otherwise. There were people to whom they could direct complaints, people who (thanks to their role as customers) players felt entitled to critique and direct. The division between those who had access to the software code and those who did not remained strong and important, but the authority that developers had was tempered by a careful scepticism on the part of customers watchful for possible exploitation.

There were also some less positive aspects to players' relationships with TERA's management: players tended to be impatient, short-sighted and primarily interested in immediate gratification. Despite showing evidence of creativity and intelligence in 
the course of playing the game, TERA players tended to want developers to 'fix' their problems in-game rather than to find their own solutions. Nonetheless, players were able to interact with and strongly influence the forces which defined their reality, because those forces were human beings. As biosociality (and perhaps cybersociality) become more prominent in contemporary life, it will be important to acknowledge that in many cases the structuring elements in our cultures will originate with people (or corporations). These people will have their own motivations and roles in society, and it will be possible to interact with and affect them through human mechanisms such as exchange. Forms of agency which influence those who control and maintain vital technological structures will become important for those who are unable to operate those technologies for themselves. TERA players used the resources at their disposal (learned from the virtual and physical worlds) to act within and use this new kind of structure, establishing channels through which they could exert agency and express themselves despite their limited ability to directly affect the virtual world. 


\section{Glossary}

Class: in TERA, a choice made at character creation defining what combat style, weapons and armour a character will be able to use. Each class had a unique style of play and different abilities available to it. TERA had eight classes: warrior, lancer, mystic, priest, sorcerer, archer, slayer, and berserker.

DPS: 'damage per second', the shorthand used for the damage-dealing role in combat or for characters specialising in this role. In TERA, sorcerers, slayers, berserkers, archers, and often warriors were DPS characters.

Healer: one of the three main combat roles. Healers were responsible for restoring the health of allies who took damage, but were relatively vulnerable and could not deal much damage. In TERA, priests and mystics were healer characters.

Instance: a kind of gameplay which occurred in an area separated off from the shared game world. Each group that entered an instance had their own copy of the zone, which existed for as long as they were there. Other players could not intrude on or help in an instance they were not part of, but multiple groups could attempt an instance at the same time (within their own separate versions of the area). Colloquially, major instances were also known as dungeons.

MMORPG: 'massively multi-player online role-playing game'. This term signifies online games with a persistent, shared virtual world where players can encounter each other, and where they develop one or more characters as their representations in that world, through levelling up and customisation.

'Open world': anything which occurred in the shared zones of TERA's game world, such as guild warfare, open world PvP, and questing; contrasted to instanced content.

PvE: 'player versus environment', as opposed to PvP. Anything that pitted players against programmed or computer-generated enemies was PvE. PvE servers did not allow open world PvP. 
PvP: 'player versus player', the term used for any combat or competition in which players fought other players rather than computer-generated enemies. This could take the form of duels, deathmatches, or later battlegrounds (all of which required mutual consent from participants), or open world PvP (which was allowed only on designated PvP servers).

Race: in TERA, a choice made at character creation defining a character's basic appearance and some minor abilities. Races in TERA were more like species than ethnicities, with very distinct physical features. The options were human, castanic, baraka, elin, high elf, popori and amani.

Server: either the computers where the data that comprises a game world is stored, which players must connect to in order to play; or a version or copy of the game world where a character is created and situated, such as Sienna Canyon server. Servers in this sense are separated because the hardware can only support so many players in one copy of the world at once.

Tank: one of the three main combat roles; a defensive character whose job was to place themselves in harm's way to prevent other characters from taking damage. In TERA the lancer was the main tank class, with warriors intended as 'secondary tanks'.

WoW: World of Warcraft, an online game commonly referred to by its acronym. It was by far the largest English language MMORPG at the time of writing (despite losing a significant number of subscribers in recent years) and was the benchmark by which new games tended to be judged. 


\section{Bibliography}

Achterbosch, Leigh, Robyn Pierce and Gregory Simmons (2008) 'Massively Multiplayer Online Role-Playing Games: The Past, Present and Future.' ACM Computers in Entertainment 5(4). DOI 10.1145/1324198.1324207.

Ahearn, Laura (2001) 'Language and Agency.' Annual Review of Anthropology 30: 109-137.

American Anthropological Association (2012) 'Statement on Ethics: Principles of Professional Responsibility.' http://www.aaanet.org/coe/Code_of_Ethics.pdf (accessed 29/01/13).

Anderson, Elizabeth S. (1999) ‘What Is the Point of Equality?' Ethics 109(2): 287337 .

Ang, Chee Siang, and Panayiotis Zaphiris (2010) 'Social Roles of Players in MMORPG Guilds.' Information, Communication \& Society 13(4): 592-614.

Applbaum, Kalman (1998) 'The Sweetness of Salvation: Consumer Marketing and the Liberal-Bourgeois Theory of Needs.' Current Anthropology 39(3): 323-350.

Bainbridge, William Sims (2010) 'Online Multiplayer Games.' Synthesis Lectures on Information Concepts, Retrieval, and Services 13. DOI: 10.2200/Soo232ED1Vo1Y200912ICR013.

Bell, Mark W. (2008) 'Toward a Definition of "Virtual Worlds."”' Journal of Virtual Worlds Research 1(1). DOI: 10.4101/jvwr.v1i1.283.

Blanden, Jo, Paul Gregg and Stephen Machin (2005) 'Intergenerational Mobility in Europe and North America.' London School of Economics, Centre for Economic Performance.

Blanke, David (2007) 'Consumer Choice, Agency, and New Directions in Rural History.' Agricultural History 81(2): 182-203. 
Boellstorff, Tom (2008) Coming of Age in Second Life: An Anthropologist Explores the Virtually Human. Princeton: Princeton University Press.

Boellstorff, Tom, Bonnie Nardi, Celia Pearce and T. L. Taylor (2012) Ethnography and Virtual Worlds: A Handbook of Method. Princeton: Princeton University Press.

Briggs, Jean (1970) Never in Anger: Portrait of an Inuit Family. Cambridge: Harvard University Press.

Business Wire (2010) 'En Masse Entertainment Debuts as New MMO Game Company.' http://www.businesswire.com/news/home/20100224005268/en/EnMasse-Entertainment-Debuts-MMO-Game-Company (accessed 14/o1/13).

Butler, Brian, Lee Sproull, Sara Kielser and Robert Kraut (2002) 'Community Effort in Online Groups: Who Does the Work and Why?' In Suzanne Weisband ed. Leadership at a distance: Research in Technologically Supported Work. NY: Lawrence Erlbaum Associates.

Caillois, Roger (1961 [1958]) Man, Play and Games (Meyer Barash trans.) New York: The Free Press of Glencoe, Inc.

Castronova, Edward (2001) 'Virtual Worlds: A First-Hand Account of Market and Society on the Cyberian Frontier.' The Gruter Institute Working Papers on Law, Economics, and Evolutionary Biology 2(1) http://www.bepress.com/giwp/default/vol2/iss1/art1 (accessed 05/02/13).

Crutzen, Paul and Eugene F. Stoermer (2000) 'The “Anthropocene”.' IGBP Global Change Newsletter 41: 17-18.

Csikszentmihalyi, Mihaly (1990) Flow: The Psychology of Optimal Experience. New York: Harper and Row.

Csikszentmihalyi, Mihaly and Stith Bennett (1971) 'An Exploratory Model of Play.' American Anthropologist 73(1): 45-58. 
Debeauvais, Thomas, Bonnie Nardi, Diane J. Schiano, Nicolas Ducheneaut and Nicholas Yee (2011) 'If You Build It, They Might Stay: Retention Mechanisms in World of Warcraft.' Proceedings of the $6^{\text {th }}$ International Conference on Foundations of Digital Games: 180-187. DOI: 10.1145/2159365.2159390.

De Certeau, Michel (1984) The Practice of Everyday Life. Berkley: University of California Press.

Delucchi, Michael and Kathleen Korgen (2002) “'We're the Customer - We Pay the Tuition”: Student Consumerism among Undergraduate Sociology Majors.' Teaching Sociology 30(1): 100-107.

Dibbell, Julian (1998) My Tiny Life: Crime and Passion in a Virtual World. New York: Henry Holt and Company.

Donath, Judith S. (1999) 'Identity and Deception in the Virtual Community' In Smith, Marc A. and Peter Kollock, eds. Communities in Cyberspace, pp. 27-57. London: Routledge.

Ducheneaut, Nicolas, Nicholas Yee, Eric Nickell, and Robert J. Moore (2006) “"Alone Together?” Exploring the Social Dynamics of Massively Multiplayer Online Games.' CHI 'o6 Proceedings of the SIGCHI Conference on Human Factors in Computing Systems: 407-416. DOI: 10.1145/1124772.1124834.

Ducheneaut, Nicolas, Nicholas Yee, and Victoria Beltotti (2010) 'The Best of Both (Virtual) Worlds: Using Ethnography and Computational Tools to Study Online Behaviour.' Ethnographic Praxis in Industry Conference Proceedings: 136-148. DOI: 10.1111/j.1559-8918.2010.00013.x.

En Masse Entertainment (2010) TERA Official Website, http://tera.enmasse.com. Fekete, John, ed. (1984) The Structural Allegory: Reconstructive Encounters with the New French Thought. Manchester: Manchester University Press. 
Fletcher, James (2012) 'Sexual harassment in the world of video gaming.' $B B C$ World Service. http://www.bbc.co.uk/news/magazine-1828000o (accessed 23/11/12).

Foster, Robert J. (2007) 'The Work of the New Economy: Consumers, Brands, and Value Creation.' Cultural Anthropology 22(4): 707-731.

Giddens, Anthony (1984) The Constitution of Society: Outline of the Theory of Structuration. Cambridge: Polity Press.

Glickman, Lawrence B. (2009) Buying Power: A History of Consumer Activism in America. Chicago: University of Chicago Press.

Graham, Kernie Lewis (2010) 'Virtual Playgrounds? Assessing the Playfulness of Massively Multiplayer Online Role-Playing Games.' American Journal of Play 3(1): 106-125.

Gruneau, Richard S. (1980) 'Freedom and Constraint: The Paradoxes of Play, Games and Sports.' Journal of Sport History 7(3): 68-86.

Henricks, Thomas (2008) 'The Nature of Play: An Overview.' American Journal of Play 1(2): 157-180.

Hine, Christine (2000) Virtual Ethnography. Los Angeles: SAGE Publications.

Huizinga, Johan (1950) Homo Ludens: A Study of the Play-Element in Culture. Boston: Beacon Press.

Jacobs, Meg (2011) 'State of the Field: The Politics of Consumption.' Reviews in American History 39(3): 561-573.

Jakobsson, Mikael and T. L. Taylor (2003) 'The Sopranos meets Everquest - Social networking in massively multiuser networking games.' Melbourne DAC, the $5^{\text {th }}$ International Digital Arts and Culture Conference: 81-90. http://hypertext.rmit.edu.au/dac/papers/Jakobsson.pdf (accessed 01/05/12). 
Jakobsson, Mikael (2006) 'Questing for Knowledge - Virtual Worlds as Dynamic Processes of Social Interaction.' In Ralph Schroeder and Ann-Sofie Axelsson, eds. Avatars at Work and Play: Collaboration and Interaction in Shared Virtual Environments, pp. 209-225. Netherlands: Springer.

Johnson, Neil F, Chen Xu, Zhenyuan Zhao, Nicolas Ducheneaut, Nicholas Yee, George Tita and Pak Ming Hui (2009) 'Human group formation in online guilds and offline gangs driven by a common team dynamic.' Physical Review E 79(6): 066117.

Kozinets, Robert V. and Jay M. Handelman (2004) 'Adversaries of Consumption: Consumer Movements, Activism and Ideology.' Journal of Consumer Research 31: 691-704.

Lee, Jungki (2010) 'Perceived power imbalance and customer dissatisfaction.' The Service Industries Journal 30(7): 1113-1137.

Malaby, Thomas M.

(2009a) 'Anthropology and Play: The Contours of Playful Experience.' New Literary History 40: 205-218.

(2009b) Making Virtual Worlds: Linden Lab and Second Life. Ithaca, NY: Cornell University Press.

Markham, Annette N. (1998) Life Online: Researching Real Experience in Virtual Space. Walnut Creek, CA: Altamira Press.

Miller, Daniel and Don Slater (2000) The Internet: An Ethnographic Approach. Oxford: Berg.

Miller, Stephen (1973) 'Ends, Means, and Galumphing: Some Leitmotifs of Play.' American Anthropologist, New Series 75(1): 87-98.

Mol, Annemarie (2008) The Logic of Care: Health and the Problem of Patient Choice. London: Routledge. 
Montola, Markus (2012) 'Social Constructionism and Ludology: Implications for the Study of Games.' Simulation \& Gaming 43(3): 300-320.

Morningstar, Chip and F. Randall Farmer (1991) 'The Lessons of Lucasfilm's Habitat.' In Michael Benedikt, ed. Cyberspace: First Steps, pp. 273-301. Cambridge: MIT Press.

Nardi, Bonnie (2010) My Life As A Night Elf Priest: An Anthropological Account of World of Warcraft. Ann Arbor: University of Michigan Press.

Newzoo (2012) 'The Global MMO Market: Sizing and Seizing Opportunities.' http://www.newzoo.com/infographics/the-global-mmo-market-sizing-and-seizingopportunities (accessed 14/12/12).

O’Reilly, Karen (2009) Key Concepts in Ethnography. London: SAGE Publications.

Ortner, Sherry B. (2006) Anthropology and Social Theory: Culture, Power, and the Acting Subject. Durham: Duke University Press.

Papargyris, Anthony and Angeliki Poulymenakou (2009) 'The Constitution of Collective Memory in Virtual Games Worlds.' Journal of Virtual Worlds Research 1(3). DOI: 10.4101/jvwr.v1i3.342.

Pearce, Celia (2009) Communities of Play: Emergent Cultures in Multiplayer Games and Virtual Worlds. Cambridge: MIT Press.

Putnam, Robert (2000) Bowling Alone: The Collapse and Revival of American Community. New York: Simon \& Schuster.

Rabinow, Paul (1999) 'Artificiality and Enlightenment: From Sociobiology to Biosociality.' In Mario Biagioli, ed. The Science Studies Reader, pp. 407-416. London: Routledge. 
Rapport, Nigel (2000) 'The narrative as fieldwork technique: processual ethnography for a world in motion.' In Vered Amit, ed. Constructing the Field: Ethnographic Fieldwork in the Contemporary World, pp. 71-95. London: Routledge.

'Ravious' (2012) 'MMO Baby Fat.' Kill Ten Rats.

http://www.killtenrats.com/2012/o8/16/mmo-baby-fat (accessed 30/o1/13).

Royce, Brianna (2013) 'Ask Massively: Developers, players and respect.' Massively. http://massively.joystiq.com/2013/o1/31/ask-massively-developers-players-andrespect (accessed 31/01/13).

Sahlins, Marshall

(1981) Historical Metaphors and Mythical Realities: Structure in the Early History of the Sandwich Islands Kingdom. Ann Arbor: University of Michigan Press.

(1982) 'Individual Experience and Cultural Order.' In William H. Kruskal ed. The Social Sciences: Their Nature and Uses, pp.35-48. Chicago: University of Chicago Press.

Sanders, Benjamin, Paul Dowland, Shirley Atkinson, and Steve Furnell (2011) 'Massively Multi-Player Online Role Playing Games: What's the Risk?' Journal of Virtual Worlds Research 3(3). DOI: 10.4101/jvwr.v3i3.874.

Scott, James C. (1985) Weapons of the Weak: Everyday Forms of Peasant Resistance. New Haven: Yale University Press.

Seay, A. Fleming, William J. Jerome, Kevin Sang Lee and Robert E. Kraut (2004) 'Project Massive: A Study of Online Gaming Communities.' CHI 'O4 Extended Abstracts on Human Factors in Computing Systems: 1421-1424. DOI: 10.1145/985921.986080.

Sewell, William H. Jr. (1992) 'A Theory of Structure: Duality, Agency and Transformation.' American Journal of Sociology 98(1): 1-29. 
Snodgrass, Jeffrey G., H. J. François Dengah II, Michael G. Lacy, Jesse Fagan, David Most, Michael Blank, Lahoma Howard, Chad R. Kershner, Gregory Krambeer, Alissa Leavitt-Reynolds, Adam Reynolds, Jessica Vyvial-Larson, Josh Whaley, and Benjamin Wintersteen (2012) 'Restorative Magical Adventure or Warcrack? Motivated MMO Play and the Pleasures and Perils of Online Experience.' Games and Culture $7(1): 3-28$.

Steinkuehler, Constance A. (2004) 'Learning in Massively Multiplayer Online Games.' Proceedings of the $6^{\text {th }}$ International Conference on Learning Sciences: 521- $^{2}$ 528.

Steinkuehler, Constance A. and Dmitri Williams (2006) 'Where Everybody Knows Your (Screen) Name: Online Games as “Third Places.”' Journal of ComputerMediated Communication 11(4): 885-909.

Stenros, Jaakko and Olli Sotamaa (2009) 'Commoditization of Helping Players Play: Rise of the Service Paradigm.' Breaking New Ground: Innovation in Games, Play, Practice and Theory; Proceedings of DiGRA 2009. http://www.digra.org/dl/db/o9287.24201.pdf (accessed o8/o1/13).

Stewart, Alex (1998) The Ethnographer's Method. London: SAGE Publications.

Sturrock, John (1986) Structuralism. London: Paladin, Grafton Books.

Suler, John

(1996) 'One of Us: Participant Observation Research at the Palace.' The Psychology of Cyberspace. http://wwwusr.rider.edu/ suler/psycyber/partobs.html (accessed 07/01/13).

(2000) 'Ethics in Cyberspace Research: Consent, Privacy and Contribution.' The Psychology of Cyberspace. http://www-usr.rider.edu/ suler/psycyber/ethics.html (accessed o8/o1/13). 
Taylor, T.L.

(1999). 'Life in Virtual Worlds: Plural Existence, Multimodalities, and Other Online Research Challenges.' American Behavioral Scientist 43(3): 436-449.

(2003) "Intentional Bodies: Virtual Environments and the Designers Who Shape Them," International Journal of Engineering Education, 19 (1): 25-34.

(2006a) 'Does WoW Change Everything? How a PvP Server, Multinational Player Base, and Surveillance Mod Scene Caused Me Pause.' Games and Culture 1(4):1-20.

(2006b) Play Between Worlds: Exploring Online Game Culture. Cambridge: MIT Press.

Turkle, Sherry (1995) Life on the Screen: Identity in the Age of the Internet. New York: Touchstone.

Turner, Victor, with Edith L. B. Turner ed. (1985) On The Edge of the Bush: Anthropology as Experience. Tucson: University of Arizona Press.

Williams, Dmitri, Nicolas Ducheneaut, Li Xiong, Yuanyuan Zhang, Nick Yee and Eric Nickell (2006) 'From Tree House to Barracks: The Social Life of Guilds in World of Warcraft.' Games and Culture 1(4): 338-361.

Williams, Dmitri, Scott Caplan and Li Xiong (2007) 'Can You Hear Me Now? The Impact of Voice in an Online Gaming Community.' Human Communication Research 33(4): 427-449.

Yee, Nick (2006a) 'The Labor of Fun: How Video Games Blur the Boundaries of Work and Play.' Games and Culture 1(1): 68-71.

Yee, Nick (2006b) 'The Psychology of Massively Multi-User Online Role-Playing Games: Motivations, Emotional Investment, Relationships and Problematic Use.' In Ralph Schroeder and Ann-Sofie Axelsson, eds. Avatars at Work and Play: 
Collaboration and Interaction in Shared Virtual Environments, pp. 187-207. Netherlands: Springer.

Young, Michael (1958) The Rise of the Meritocracy. Harmondsworth: Penguin Books.

Zabban, Vinciane (2011) 'What Keeps Designers and Players Apart? Thinking How an Online Game World is Shared.' Proceedings of DiGRA 2011 Conference: Think Design Play. http://www.digra.org/dl/db/11312.58594.pdf (accessed 08/01/12).

\section{Games}

ArenaNet (2012) Guild Wars 2.

Bethesda Game Studios (2012) The Elder Scrolls V: Skyrim.

BioWare (2011) Star Wars: The Old Republic.

Blizzard Entertainment

(2004) World of Warcraft.

(2012) Diablo III.

En Masse Entertainment (2012) TERA: The Exiled Realm of Arborea.

FunCom (2012) The Secret World.

NCsoft (1998) Lineage. 